KERNFORSCHUNGSANLAGE JULICH GESELLSCHAFT MIT BESCHRANKTER HAFTUNG Institut für Fesłkörperforschung

Phonons and Librons in Solid Hydrogen and Deuterium

by

Franz-Georg Mertens

Jül - 734 - FF

Februar 1971

$+=$ 


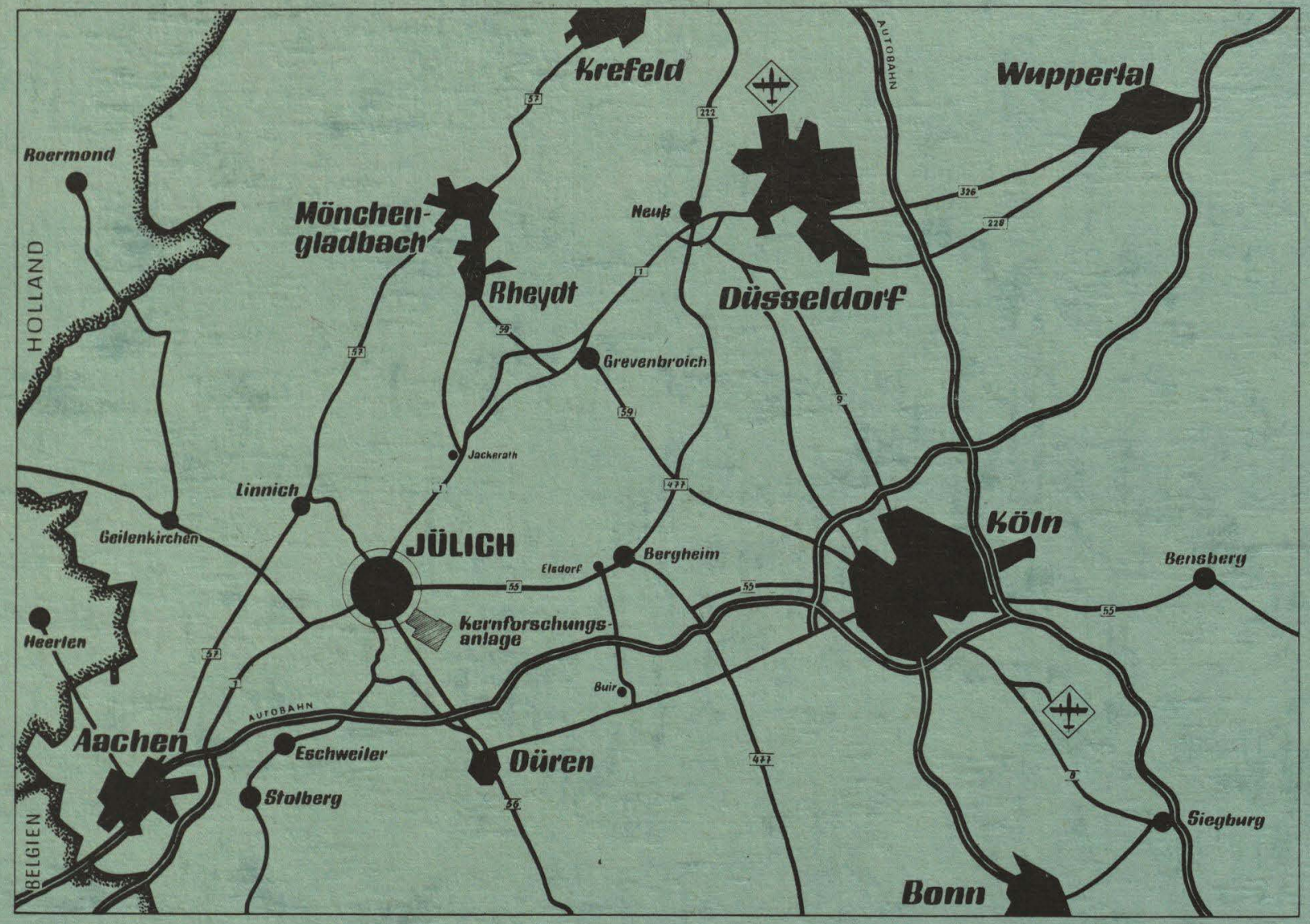

Berichte der Kernforschungsanlage Jülich - Nr. 734

Institut für Festkörperforschung Jül - 734 - FF

\author{
Dok.: Hydrogen (Solid) - Phonons \\ Hydrogen (Solid) - Librons \\ Deuterium (Solid) - Librons \\ Deuterium (Solid) - Phonons
}

Im Tausch zu beziehen durch: ZENTRALBIBLIOTHEK der Kernforschungsanlage Jülich GmbH, Jülich, Bundesrepublik Deutschland 


\section{Phonons and Librons in Solid Hydrogen and Deuterium}

by

Franz-Georg Mertens

D 82 (Diss. T.H. Aachen) 
A Introduction

B Ground state

I Ground state structure and trial

II Cluster expansion of the ground state energy and choice of a 2-particle short range correlation function

III The interaction between the molecules

IV Results of the ground state variation

C Collective excitations

V Random Phase Approximation

VI Effective Hamiltonian for systems

VII Calculation of matrix elements 65

VIII Solution of the secular equation 75

IX Dispersion curves and spectrum of the phonons 90

D Appendices

$X$ Series expansion of integrals

XI Special matrix elements 
A Introduction

There are at least three reasons why it is interesting to consider solid hydrogen $\left(H_{2}\right)$ and deuterium $\left(D_{2}\right)$.

1) These solids are quantum crystals (like helium); this means that the displacements of the molecules due to zero point and thermal motion are not small compared with the lattice parameter. Therefore the usual harmonic theory for lattice dynamics cannot be applicated.

2) $H_{2}$ and $D_{2}$ are the only known crystals in which the molecules rotate nearly freely. The energy differences between different angular momentum states $\mathcal{J}$ are large compared with the temperature region in which the solids exist at normal pressure. (e.g. $850^{\circ} \mathrm{K}$ between $\mathrm{J}=3$ and $\mathrm{J}=1$ for ortho- $\mathrm{H}_{2}$ and $510^{\circ} \mathrm{K}$ between $\mathrm{J}=2$ and $\mathrm{J}=0$ for $\operatorname{para}-\mathrm{H}_{2}$ ).

3) The intermolecular interactions are rather well known, so the theories do not contain free parameters and need not be fitted to experiments.

Both $H_{2}$ and $D_{2}$ crystallize at normal pressure at $\sim 15^{\circ} \mathrm{K}$ in the hcp structure. At these low temperatures only the lowest rotational levels are populated: $\mathrm{J}=1$ for ortho-hydrogen $\left(0-\mathrm{H}_{2}\right)$ and para-deuteriur $\left(p-D_{2}\right)$ and $J=0$ for $p-H_{2}$ and $o-D_{2}$.

Crystals containing more than a minimum concentration $(\sim 608)$ of the odd-J species show a $\lambda$-anomaly in the specific heat at a temperature $T_{c}$, which increases nearly linearly with the concentration.

$T_{c}$ is extrapolated to $2.8^{\circ} \mathrm{K}$ and $3.8^{\circ} \mathrm{K}$ for pure $0-\mathrm{H}_{2}$ and

High purity $(\sim 998)$ can be obtained by using the preferential adsorption of one species by aluminium oxide [28] . 
$\mathrm{P}-\mathrm{D}_{2}$ respectively $[12-14]$.

The $\lambda$-anomaly has been explained by a first order phase transition. Above $T_{c}$ the three $m_{y}$ levels $(0, \pm 1)$ are degenerate and the crystal is orientationally disordered. Below $T_{c}$ the anisotropic electrical quadrupole-quadrupole $(q-q)$ interactions cause the angular momentum vectors of the molecules to prefer certain orientations with respect to the crystalline axes, and the degeneracy of the $m_{y}$ levels is lifted.

$x$-ray and neutron diffraction experiments [7-11] have shown that the orientational transition is accompanied by a change of the crystal structure from hcp to fcc.

Theories, based on a rigid lattice model, have predicted a Paj space group for the ordered ground state. This structure has been found first in a classical calculation for $T=0$ by considering arrays of linear quadrupoles $[1,2]$. Lateron the phase transition has been calculated in the molecular field approximation (or Bragg-Williams method) [3-6]. Moreover it has been shown that the $\mathrm{Pa} 3$ structure is consistent with the neutron diffraction pattern of $\mathrm{p}-\mathrm{D}_{2}[10,11]$.

In the Pa3 space group there are four molecules per cubic unit cell. Each molecule belongs to one of the four equivalent simple cubic sublattices of the fcc structure. When we associate one of the four body diagonals as quantization axis to each sublattice we can characterize the Bragg-Williams ground state very easily: Each molecule is in the $m_{j}=0$ state with respect to the quantization axis of its sublattice.

In the molecular field method the states $m_{j}= \pm 1$ describe degenerate localized excitations. In a better approximation the molecular field must be considered to be time dependent ( $t$ ime dependent Hartree approximation or Random Phase Appr. (RPA)). Then it can be seen that the low lying excitations are of collective nature (like spin waves in antiferromagnetic materials). 
The theory of these angular momentum or librational waves or librons has been developed by several authors [15-19] using different mathematical methods but the same physical approaches and yielding the same results in the end: The libron excitations form a band (width $\sim 1.3 \mathrm{meV}$ for $0-\mathrm{H}_{2}$ and $\sim 1.6 \mathrm{meV}$ for $\mathrm{p}-\mathrm{D}_{2}$ ), which is separated from the ground state by an energy gap of $\sim 0.9$ resp. 1.1 mev. Here the $q-q$-coupling constant $\Gamma_{0}$ of the rigid lattice has been used.

Later on the existence of librons in $0-\mathrm{H}_{2}$ and $\mathrm{p}-\mathrm{D}_{2}$ has been proved experimentally for the limit of long wavelengths $(\underline{k}=0)$ by Raman scattering $[20,21]$. The results agree very well with the theory, if $\Gamma_{0}$ is replaced by an effective $q-q-c o u p l i n g ~ c o n s t a n t$ $\Gamma_{0}$ eff , which has been measured by quite a lot of experiments [see 22] and can also be obtained by theoretical calculations [23]. Moreover it is necessary to include the large anharmonic libronlibron interactions into the calculations [24].

For $k=0$ the libron theory seems to be in full agreement with the experiment. As to $k \neq 0$ it has been proposed $[16,18,19]$ that the dispersion curves and the spectrum be measured by scattering of slow neutrons. Such work is in progress [25].

Consequently the next theoretical step should be to calculate the phonons and to include the phonon-libron coupling. This coupling occurs for small $\mathrm{k}$ between the acoustical phonons and all libron modes. See the rough estimate in $[18,19]$. Furthermore the existing phonon theories $[26,27]$ neglect the $q-q$-forces and the orientational order of the molecules. They use the division into four simple cubic sublattices only formally and probably cannot explain the number of optical phonon lines which have been observed by Raman scattering [26].

Therefore the purpose of this work is to develop within the frame of the RPA for $T=0$ a theory which describes all low-lying collective excitations (phonons, librons, and "mixed" excitations, which arise because of the coupling) in pure $0-\mathrm{H}_{2}$ and $\mathrm{p}-\mathrm{D}_{2}$. 
Because these solids are quantum crystals, the problem is rather complicated compared with normal crystals: The short range correlations (SRC) between the molecules must be taken into account. This is made by introducing a two particle SRC function (Jastrow factor) into the wavefunction of ground and excited states.

In part B the energy of the ordered ground state is written as a cluster expansion [29 - 32] . The variational parameters contained in the wavefunction are determined by minimizing the first two terms of the expansion. The convergence of the expansion is di scussed in detail.

In part $C V$ the above mentioned RPA is first developed for systems witout SRC, using the method of time dependent density matrices [33] - In C VI an effective Hamiltonian for systems with SRC, which has been derived by a generalized cluster expansion for excited states [34] , is inserted into the RPA. All matrix elements which appear in the RPA are calculated in C VII.

$C$ VIII shows that there is no coupling between librons and phonons for $k=0$. For this case the secular equation is solved analytically and the results are compared with optical measurements $[26,21]$.

For $k \neq 0$ the numerical evaluation of the theory is carried out in this work only for the case of phonons in the orientationally ordered crystals (C IX). Iibrons have been calculated separately in [18, 19]. The coupled system of librons and phonons will be calculated in second paper. Since the matrix elements for this work are known from $C$ VII, only some numerical work has to be done.

In the whole paper the disordered hcp phase has been considered, too. This phase has its own interest and can be used as a good test for the phonon part of the theory (especially concerning the SRC), since for the hap phase there exist already detailed neutron diffraction experiments $[35,36]$ and also optical measurements [21]. 


\section{B Ground State}

I Ground state structure and trial wavefunction

The ordered ground state is approcimately described by the wavefunction

$$
\phi_{0}=\prod_{i}^{N} \psi_{i} \cdot \frac{N}{\prod_{i \alpha_{j}} f_{i j}}
$$

Short range correlations (SRC) arise because of the hard core of the intermolecular potential and are taken into account by the SRC-function $f_{i j}$, which depends oniy on the distance $t=T_{i j}=\left|\underline{R}_{i}-\underline{R}_{i}\right|$ between the molecules and shall prevent them from coming close together.

This means we must choose a function $f_{i j}$, which tends very quickly to zero, if $r$ becomes smaller than the hard core distance. For large distances $f_{i j}$ must go to 1. In chapter II special forms of $f_{i j}$, containing free parameters, are discussed.

The one particle function $\Psi_{i}^{0}$ in the case of $\mathrm{O}-\mathrm{H}_{2}$ and $p-D_{2}$ depends on the position $\underline{R}_{i}$ and the orientation $\Omega_{i}=\left(\vartheta_{i}, \varphi_{i}\right)$ of the molecule $i$. We have to consider only the lowest rotational state $\gamma=1$ and can expand $\psi_{i}^{\circ}$ in this space:

$$
\psi_{i}^{0}=\sum_{m} Y^{m}\left(\underline{R}_{i}\right) \cdot Y_{1, m}\left(\ell_{i}\right) ; \quad m=m y=0, \pm 1
$$

where $Y_{1, m}$ are normalized spherical harmonics.

Using real functions we get

$$
\psi_{i}^{0}=\sum_{k} \varphi^{k}\left(R_{i}\right) Y_{k}\left(\Omega_{i}\right) \quad ; k=x, y, z
$$


with

$$
\begin{aligned}
& y_{x}=\frac{1}{\sqrt{2}}\left(-y_{1,1}+y_{1,-1}\right) ; \quad \varphi^{x}=\frac{-1}{\sqrt{2}}\left(\varphi^{1}-\varphi^{-1}\right) \\
& y_{y}=\frac{i}{\sqrt{2}}\left(Y_{1,1}+y_{1,-1}\right) ; \quad \varphi^{y}=\frac{-i}{\sqrt{2}}\left(\varphi^{1}+\varphi^{-1}\right) \\
& y_{z}=y_{1,0} \quad ; \quad \varphi^{z}=\varphi^{0}
\end{aligned}
$$

The libron theories [15-19] assumed the Pa 3 structure and a rigid lattice, which means here

$$
\varphi^{0}=\delta\left(\underline{R}_{i}-\underline{R}_{i}^{0}\right) ; \quad \varphi^{\stackrel{x}{y}}=0
$$

Since these theories have been successfull, we are allowed to consider $\varphi^{\circ}\left(\underline{R}_{i}\right)$ to be localized around the mean position $\underline{R}_{i}^{\circ}$ of the molecule $i$ and $\varphi_{y}^{x}$ to be small comparea with $\varphi^{\circ}$.

More detailed information about $\varphi_{y}^{x}$ is obtained by using the point symmetry of the lattice sites $\underline{R}_{i}^{0}$. This leads to nodal planes

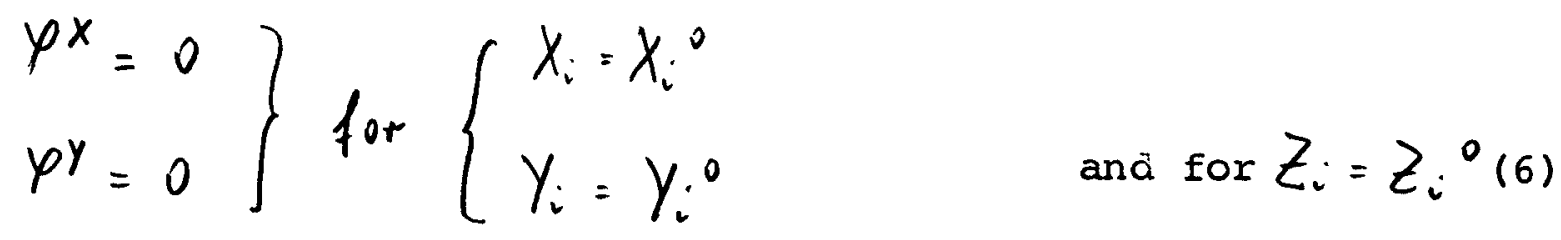

with $\underline{R}_{i}=\left(X_{i}, Y_{i}, Z_{i}\right)$.

Otherwise $\varphi^{x}$ and $\varphi y$ must go to zero, where $\varphi^{0}$ vanishes. Therefore $\varphi^{x}$ and $\varphi y$ seem to be small functions compared to $\varphi^{\circ}$, and we approximate $\psi_{i}{ }^{\circ}$ by 


$$
\psi_{i}^{0}=\varphi^{0}\left(\underline{R}_{i}-\underline{R}_{i}^{0}\right) \cdot Y_{1,0}\left(\Omega_{i}\right)
$$

and assume the Pa3 structure.

A better approximation would be to use functions of the form

$$
\begin{aligned}
& \varphi^{x}=A \cdot\left(X_{i}-X_{i}^{0}\right) \cdot\left(Z_{i}-Z_{i}^{0}\right) \cdot \varphi^{0}\left(\underline{R}_{i}-\underline{R}^{0}\right) \\
& \varphi^{Y}=A \cdot\left(Y_{i}-Y_{i}^{0}\right) .
\end{aligned}
$$

with $A$ a parameter.

We now introduce an explicite notation for the description of the Pa3 structure:

The fcc lattice is devided into four equivalent simple cubic sublattices.

$$
\underline{R}_{i}^{0}=a(\underline{m}+\underline{s}) ; i \equiv(\underline{m}, g)
$$

with $\underline{m}=\left(m_{1}, m_{2}, m_{3}\right)=$ index of cubic cell

$g=$ index of the sublattice

$\mathrm{a}=$ lattice constant

$\underline{s}_{g}=$ position inside of the cell

$$
S_{1}=0 ; \quad S_{2}=\frac{1}{2}[110] ; \quad S_{3}=\frac{1}{2}[011] ; \quad S_{4}=\frac{1}{2}[101]
$$

All molecules of each sublattice are in the state $m=0$ with respect 
$-8-$

to one of the four body diagonals of the fcc lattice. Each sublattice is associated with one of the four body diagonals. As is shown in [19], there exist two equivalent associations. We choose one of them:

$$
\underline{d}_{1}=[111] ; \underline{d}_{2}=[\overline{1} 11] ; \underline{d}_{3}=[1 \overline{1} 1] ; \underline{d}_{4}=[11 \pi]
$$

For $\mathrm{p}-\mathrm{H}_{2}$ and $\mathrm{O}-\mathrm{D}_{2}$ with hap structure the situation is much simpler. All molecules are in the $\gamma=0$ state, which is spherically cymetrice.

Thereby

$$
\psi_{i}^{0}=\varphi^{0}\left(\underline{R}_{i}-\underline{R}_{i}^{0}\right)
$$

The hop lattice is described by

$$
\underline{R}^{0}=a\left(\underline{\ell}+\underline{s}_{v}\right) ; \quad \nu=1.2
$$

$$
\text { with } \begin{aligned}
\underline{l} & =\left(l_{1}-\frac{1}{2} l_{2}, \frac{\sqrt{3}}{2} l_{2}, c l_{3}\right) ; l_{1}, l_{2}, l_{3}=0,1,2, \ldots \\
\underline{S}_{1} & =0, \\
\underline{s}_{2} & =\left(\frac{1}{2}, \frac{\sqrt{3}}{6}, \frac{c}{2}\right), \\
a & =\text { lattice constant, } \\
c & =2 \frac{\sqrt{2}}{\sqrt{3}} .
\end{aligned}
$$


II Cluster expansion of the ground state energy and choice of a 2-particle short range correlation function

Let us first remind of the method and the result of Nosanow and coworkers for helium [29-32].

Their trial wavefunction for the ground state is, similar to (I,1):

$$
\phi_{0}=\prod_{i} \varphi^{0}\left(1 \underline{R}_{i}-\underline{R}^{0}{ }^{0}\right) \prod_{i<j} f_{i j}
$$

The ground state energy is

$$
E_{i}=\frac{\left(\phi_{0}, H \phi_{0}\right)}{\left(\phi_{0}, \phi_{0}\right)}
$$

wj.th the Hamiltonian

$$
H=\sum_{i} \frac{p_{i}^{i}}{2 M}+\sum_{i<j} V_{i j}
$$

$$
V_{i j}=\text { interatomic potential. }
$$

Nearly the whole helium work is done with a Lennard-Jones potential

$$
V(r)=4 \varepsilon\left[\left(\frac{5}{r}\right)^{12}-\left(\frac{5}{r}\right)^{6}\right]
$$

The ground state energy $E_{0}$ is expressed as a cluster expansion

$$
E_{0}=E_{01}+E_{02}+E_{03}+\ldots
$$


$-10-$

where Eon gives the contribution to the energy arising specifickly from the clustering of $n$ particles. E om vanishes, if one or more of the $n$ particles is far away from the rest of the particles in the cluster.

Except from $E_{0_{1}}$, each of the terms in (5) can be split into a kimetic energy part $E_{o m}^{\top}$ and a potential energy part $E_{0 m}^{V}$ with respect to the one particle functions $\varphi^{0}$ :

$$
E_{0}=E_{01}+E_{02}^{V}+E_{02}^{\top}+E_{03}^{v}+E_{03}^{\top}+\cdots
$$

with

$$
\begin{aligned}
& E_{01}=\sum_{i}^{\Gamma}\left\langle T\left(\underline{R}_{i}\right)\right\rangle \\
& E_{02}^{T}=\sum_{i, j}^{1}\left[\frac{\left\langle T\left(\underline{R}_{i}\right) f_{i j}^{2}\right\rangle}{\left\langle f_{i j}^{2}\right\rangle}-\left\langle T\left(\underline{R}_{i}\right)\right\rangle\right]
\end{aligned}
$$

etc

$$
\begin{aligned}
& T\left(\underline{R}_{i}\right)=-\frac{\hbar^{2}}{2 m} \nabla^{2} \ln \varphi^{0} \\
& E_{02}^{v}=\frac{1}{2} \sum_{i \cdot j}^{\prime} \frac{\left\langle\bar{V}_{i j} f_{i j}^{2}\right\rangle}{\left\langle f_{i j}^{2}\right\rangle} \\
& E_{03}^{V}=\frac{1}{2} \sum_{i, j k}^{\prime}\left[\frac{\left\langle f_{i j}^{2} f_{i k}^{2} f_{j k}^{2} \bar{V}_{i j}\right\rangle}{\left\langle f_{i j}^{2} f_{i k}^{2} f_{j k}^{2}\right\rangle}-\frac{\left\langle f_{i j}^{2} \bar{V}_{i j}\right\rangle}{\left\langle f_{i j}^{2}\right\rangle}\right]
\end{aligned}
$$

etc., where

$$
\bar{V}( \pm)=V(r)-\frac{\hbar^{2}}{2 m} \nabla^{2} \ln f(r) .
$$


$\langle\cdots\rangle$ means $\left\langle\prod_{i} \varphi_{i}{ }^{\circ}|\cdots| \prod_{i} \varphi_{i}{ }^{\circ}\right\rangle$ with normalized $\varphi_{i}^{0}$. The dash at the sums excluaies that two or more of the summation inaices are equal.

The physical interpretation of the terms is obvious: Eon is the single particle kinetic energy; $E_{02}^{V}$ is the pair interaction due to the effective potential $f^{2} \bar{V} ; E_{03}^{V}$ is the sum of all possible interactions of three particles.

The question is under which conditions and how rapidly the expansion (6) converges. Nosanow has approximated $E_{0}$ by

$$
E_{0}^{(2)}=E_{01}+E_{02}^{V}
$$

and varied this with respect to the single particle function $\varphi^{0}$ to give a differential equation for $\varphi^{\circ}$. Then for $f(r)$ a simple analytic form was chosen, containing one variational parameter $k$ :

$$
f(r)=e^{\left.-k\left[\left(\frac{5}{r}\right)^{12}-\mid \frac{5}{r}\right)^{6}\right]}
$$

This form fulfils the necessary concitions, which have been discussed in chapter $I$.

The aifferential equation for $\varphi^{0}$ was solvea numerically for different values of $K$, yielding a minimum for $E_{01}+E_{02}{ }^{V}$ with respect to $K$. Although this minimum is still far from the experimental ground state energy, it is much better than the results which had been obtainea earlier by other theories.

It turned out that $\varphi^{0}$ can be very well approximated by a Gaussian: 


$$
\varphi_{i}^{0}=\left(\frac{\gamma}{11}\right)^{3 / 4} e^{-\frac{1}{2} \gamma\left(\underline{R}_{i}-\underline{R}_{i}{ }^{0}\right)^{2}}
$$

This means that it is possible to treat the crystal to a certain extent formally as a "normal" crystal, using the harmonilc theory, if the true interaction $V(r)$ is replaced by an effective one

$$
V_{\text {eff }}=f^{2} \bar{V}=f^{2}\left[V(r)-\frac{\hbar^{2}}{2 M} \nabla^{2} \ln f(r)\right]
$$

The only difference to such a model is the denominator $\left\langle f^{2}\right\rangle$ in $E_{0_{2}}{ }^{V}$, which deviates however only by $10 \%$ from unity at most.

The next step was to calculate the leading correction terms to $E_{0}^{(2)}=E_{01}+E_{0_{2}}{ }^{v}$. This was done by replacing $\varphi_{i}{ }^{0}$ by the Gaussian (15). Then all kinetic terms Eoi vanish, except the first one

$$
E_{01}=\frac{3}{4} \frac{\hbar^{2}}{M} \gamma \cdot N
$$

This inaicates that $E_{o m}^{\top}(\mu \neq 1)$ must be small, too, when the numerical solution for $\varphi_{i}^{0}$ is used.

Then $E_{03}^{V}$ remains, which coula be obtainea by a complicated numerical procedure [32]. The result is that at the minimum of $E_{0}^{(x)}$

$E_{03}^{v}$ amounts to $1-5 \%$ of $E_{02}^{v}$ for the aifferent lattice types of helium. If $E_{03}^{V}$ is included into the variation, the variation parameters are somewhat improved $(<1 \%)$, but the ground state energy remains essentially unchanged.

rnese results indicate that for the special form of $f$ which had been chosen the cluster expansion converges rapidly. But it can be shown that the rate of convergence and even the convergence itself 
$-13-$

depend's sensitively on the form of $f$.

$f$ cannot be varied freely, because a free variation can lead into a region where the expansion no longer converges fast enough. This can easily be demonstrated by an example: If (14) is replaced by

$$
f(r)=e^{-k\left(\frac{5}{r}\right)^{12}+k^{\prime}\left(\frac{\sigma}{r}\right)^{6}} \quad ; k k^{\prime}>0
$$

the effective potential contains an attractive term $\sim k^{\prime} /+8$, which cannot be compensated by a positive $k^{\prime}$-depending term. Thus $E_{01}+E_{02}{ }^{2} \quad$ can assume very large native values, if $k^{\prime}$ is increased, and $E_{03}^{V}$ becomes large too, but positive.

Let us now consider how to use the cluster expansion technique for $H_{2}$ and $D_{2}$.

The disordered hop lattice [31] and an hypothetic disordered fcc lattice [37] have already been treated, using an isotropic Gaussian for $p^{\circ}$.

The ordered state of $0-H_{2}$ and $p-D_{2}$ is more complicated, because the single particle function $\psi_{i}^{*}$ depends on the position and orientration of the molecule.

With our ansate $(I, 7)$

$$
\psi_{i}^{0}=\varphi^{0}\left(\underline{R}_{i}-\underline{R}_{i}^{0}\right) \cdot Y_{1,0}\left(\Omega_{i}\right)
$$

we obtain

$$
T\left(\underline{R}_{i}\right)=-\frac{t^{2}}{2 M} \nabla_{\underline{R}_{i}}^{2} \ln \psi_{i}^{0}=-\frac{\hbar^{2}}{2 M} \nabla_{\underline{R}_{i}}^{2} \ln \varphi_{i}^{0},
$$


the same as before.

In the expression for $E_{01}$ the rotational energy of the molecules should be added, but it is a constant because of $j=1$ and can be left out in the variation.

The averages $\langle\cdots\rangle$ now mean

$\left\langle\prod_{i} \psi_{i}{ }^{0}\left(\underline{R}_{i}, Q_{0}\right) 1 \ldots 1 \prod_{i}^{\pi} \psi_{i}^{0}\left(\underline{R}_{i}, R_{i}\right) \quad\right.$, where the integrations must be carried out over all positions $\underline{R}_{i}$ and orientations $\Omega_{i}$.

For $\varphi_{i}^{0}$ we use the isotropic Gaussian (15). This is not only an approximation in the sense mentioned in the text in front of (15) but also because of neglecting the special point symmetry of $0-H_{2}$ and $p-\partial_{2}$. In the Pa3 space group the body diagonals are axes of threefold symmetry and should be distinguished from the directions perpenaicular to it in the ansatz for $\varphi_{i}^{0}$, for exarnple by an anisotropic Gaussian with two parameters $\gamma_{1}, \gamma_{2}$ :

$$
\varphi_{i}^{0}=\frac{\left(\gamma_{1} \gamma_{1} \gamma_{2}\right)^{1 / 4}}{\pi 3 / 4} e^{-\frac{1}{2} \underline{\mu}_{i} \Gamma \underline{\mu_{i}}}
$$

with $\quad \underline{U}_{i}=\underline{R}_{i}-\underline{R}_{i}^{0} ; \quad i \equiv(\underline{m}, g)$

and

$$
\Gamma=\left(\begin{array}{ccc}
\gamma_{1} & 0 & 0 \\
0 & \gamma_{1} & 0 \\
0 & 0 & \gamma_{2}
\end{array}\right)
$$

In each sublattice $g$ another coordinate system has to be used, where the $Z$-axis is parallel to the associated body diagonal.

But the anisotropic interactions between the molecules are small comparea with the isotropic ones (III), so the approximation (15) seems to be justified.

Though Nosanow's form of $f(r)$ (14) works remarkably well, a more 
flexible function should be tried.

An interesting behaviour of iNosanow's $f(r)$ is the slight maximum at $\sqrt[6]{2}, \sigma$, where the Lennard-Jones potential (4) has its minimum. This is explained by a positive correlation between the molecules near the hard core because of the attraction there. When one uses a function $f(r)$ without a maximum one gets poor results.

In (14) there is only one variational parameter for the two important regions: a) the hard core region $r<\sigma$, where $f(r)$ quickly goes to zero, b) the region $\tau \geqslant \sigma$, where the potential has its characteristic form. Therefore we try to separate these regions. The first iciea is to take the generalized Nosanow ansatz (18). This has the disadvantage that the position and the form of the maximum in $f(+)$ depena on each other. We try a more general ansatz:

For the region a) we take exp $\left[-k(\sigma / r)^{n}\right]$ in order to have a rapid decrease of $f$. For $b)$ we add a maximum which can be describea by a Gaussian $\alpha \exp \left[-\beta\left(r-r_{m}\right)^{2}\right]$, for example.

We neglect a possible ciependence of $f$ on the molecular orientations and take as an ansatz

$$
f(r)=e^{-k\left(\frac{\sigma}{r}\right)^{n}}+\alpha e^{-\beta\left(r-r_{m}\right)^{2}}
$$

Astonishingly enough it turns out during the variation that the parameters $k_{1} m, \beta, r_{m}$ contained in (21) can all be varied freely, only $\alpha$ cannot on the same reason as the $k^{\prime}$ in (18).

Results and further discussion concerning the choice (21) can be found in chapter IV. 
III The interaction between the molecules and the calculation of natrix elements

a) The interaction energy

The interaction between two $\mathrm{H}_{2}$ or $\mathrm{D}_{2}$ molecules in the state $\gamma=1$ is conveniently written

$$
\begin{aligned}
V_{i j}= & V_{0}\left(r_{i j}\right)+\sqrt{\frac{4 \pi}{5}} V_{1}\left(r_{i j}\right)\left\{Y_{2,0}\left(\Omega_{i}^{ \pm}\right)+Y_{2,0}\left(\Omega_{j}^{ \pm}\right)\right\} \\
& +\frac{4 \pi}{5} Y_{2}\left(r_{i j}\right) \sum_{\mu} \sigma_{\mu} Y_{2, \mu}\left(\Omega_{i}^{ \pm}\right) \cdot Y_{2, \nu \mu}\left(\Omega_{i}^{ \pm}\right)
\end{aligned}
$$

Here $\Omega_{i} I$ denotes the orientation of the molecule $i$ relative to the intermolecular axis $I_{i j}$. For the spherical harmonics $Y_{2, \mu}$ we use the phase convention of Condon and Shortly, see [38].

(1) contains the leading terms of an expansion of $V_{i j}$ in spherical harmonics. Matrix elements with the higher terms vanish, because we need in our theory only the $y=1$ and $y=0$ states.

Each $V$ in (1) consists of two parts, which result from the van der Waals and overlap forces. Moreover $V_{2}$ contains the $q-q$-interaction, due to the permanent quadrupole moment of the molecules.

For the isotropic part $V_{0}$ we use a Lennard Jones potential:

$$
V_{0}(r)=4 \varepsilon\left[\left(\frac{\sigma}{r}\right)^{12}-\left(\frac{\sigma}{r}\right)^{6}\right] ; \quad \begin{aligned}
& \varepsilon=37 \mathrm{~K}=3.188 \text { meV } \\
& \sigma=2.93 \AA
\end{aligned}
$$

The secona part in (1) depends on the orientations of the single molecliles. The distance dependent factor $V_{1}$ is not well known. We use the form 


$$
V_{1}(r)=\beta_{1} e^{-2 \alpha^{\prime}\left(r-r_{B}\right)}-\beta_{2}\left(\frac{r_{3}}{r}\right)^{6}
$$

with different sets of parameters, given in the literature. This will be discussed in detail in chapter VIII.

The third part in (1) depends on the relative orientation of the two molecules. It can be shown $[40,41]$ that in this part the electrical q-q-interaction dominates all the other interactions by about a factor of 100. These therefore can be neglectea.

Then the last term in (1) can be written [41]

$$
\Gamma\left(v_{i j}\right) \cdot Q\left(Q_{i}^{ \pm} \cdot \Omega_{i}^{ \pm}\right)=\Gamma_{i j} \cdot Q_{i j} \pm
$$

with

$$
Q_{i j}^{I}=\frac{10}{3} \pi \sqrt{70} \sum_{\mu} C\left(224_{i} \mu_{1}-\mu\right) Y_{2, \mu}\left(R_{i}^{ \pm}\right) \cdot Y_{z_{1}, \mu}\left(Q_{i}^{I}\right)
$$

The index I denotes the reference to the intermolcular axis $\Psi_{i j}$. $C$ is a Clebsch-Gordan-coefficient [38].

$$
\Gamma_{i j}(r)=\frac{6 e^{2} q^{2}}{25 r^{5}}=\Gamma_{0} \cdot\left(\frac{R_{0}}{r}\right)^{5}
$$

$\Gamma_{0}=\frac{6 e^{2} q^{2}}{25 R_{0}{ }^{5}}$ is the $q-q-$ constant of the rigid lattice, with the nearest neighbour separation $R_{0}=3.75 \AA$ for $H_{2}$ and $R_{0}=3.59 \AA$ for $D_{2}$. eq is the electrical quadrupole moment of the molecules. With $q=0.1348 \cdot 10^{-16} \mathrm{~cm}^{2} \quad$ for $H_{2}$ and $q=0.1327 \cdot 10^{-16} \mathrm{~cm}^{2}$ for $D_{2}$ [5] we use $r_{0}=0.0846 \mathrm{meV}$ for $H_{2}$ and $r_{0}=0.104$ meV for $D_{2}$. 
$-18-$

In the following we write

$$
V_{i j}=V_{i j}^{0}+V_{i j}^{1} \cdot U_{i j}^{ \pm}+\Gamma_{i j} Q_{i j}^{r}
$$

with $\quad U_{i j}^{\underline{v}}=\sqrt{\frac{4-10}{5}}\left\{\gamma_{2,0}\left(\Omega_{i}^{ \pm}\right)+Y_{2,0}\left(\Omega_{j} \pm\right)\right\}$

and $Q_{i j} \pm$ given by $(5)$.

b) The matrix elements

We want to minimize

$$
\begin{aligned}
E_{0}^{(2)} / N & =\frac{1}{N}\left(E_{01}+E_{02}{ }^{\gamma}\right) \\
& =\frac{3}{4} \frac{\hbar^{2}}{M} \gamma+\frac{1}{2} \sum_{j}^{\sum_{j}^{\prime}} \frac{\left\langle f_{i j}^{2} \bar{V}_{i i}\right\rangle}{\left\langle f_{i i}^{2}\right\rangle}
\end{aligned}
$$

by variation of the parameters $\gamma$ (contained in $\left.\varphi^{0}\right), R_{1}$ (nearest neighbour distance), $k, m, \beta, r_{m}$ (contained in the SRC-function $f$ ).

$\psi_{i}{ }^{\circ}$ is normalized. Therefore we get e.g.

$$
\left\langle f_{i j}^{2} \bar{v}_{i j}\right\rangle=\left\langle\psi_{i}^{0} \psi_{i}^{0}\left|f_{i j}^{2} \bar{V}_{i j}\right| \psi_{i}^{0} \psi_{i}^{0}\right\rangle=\left\langle 00\left|f_{i j}^{2} \bar{v}_{i j}\right| 00\right\rangle
$$

more explicitely 
$-19-$

$$
=\left\langle\varphi^{0}\left(\underline{R}_{i}\right) \cdot Y_{1,0}\left(\Omega_{i}\right) \cdot \varphi^{0}\left(\underline{R}_{1}\right) Y_{1,0}\left(R_{i}\right)\left|P_{i j}^{2} \bar{V}_{i j}\right| \varphi_{i}^{0} Y_{1,0}(i) \varphi_{i}^{0} Y_{1,0}(j)\right\rangle
$$

Two types of integrations have to be performed; the first one with respect to the position of the molecules which we call translational integration, the second with respect to the orientations of the molecules (orientational integration).

We wish to separate these integrations and see that the forms (5) and (fa) are not suitable to do so, because the orientations $\Omega_{i}, \Omega_{i}$ refer to the instantaneous intermolecular axis $\Psi_{i j}$. We therefore transform (7) to the fixed frame of the axis $I_{i j}^{0}=\underline{R}_{i}^{0}-\underline{R}_{i}^{0}$ by means of the rotation matrix $\dot{J}^{2}$, which is given e.g. in [38].

$$
Y_{2, \mu}\left(\Omega_{i} \nabla\right)=\sum_{\mu} \partial_{m, \mu}^{2}\left(\alpha, \beta_{1} \gamma\right) \cdot Y_{2, \mu m}\left(R_{i} \pm_{0}\right)
$$

Here $\alpha, \beta, \gamma$ are the Euler-angles of the rotation.

The first part in (1) is not affected by the transformation; for the second we use

$$
\begin{aligned}
& Y_{2,0}\left(R_{i}^{ \pm}\right)=\sum_{m} \partial_{m, 0}^{2}(\alpha, \beta, 0) Y_{2, m}\left(\Omega_{i}{ }^{\nu_{0}}\right) \\
& =\sqrt{\frac{4_{1 \pi}}{5}} \sum_{m} Y_{2, m}^{*}(\beta, \alpha) Y_{2, m}\left(\Omega_{i}^{*} r_{0}\right)
\end{aligned}
$$


$-20-$

Here $(\beta, \alpha)=h_{i j} \quad$ is the orientation of $r$ with respect to $Y_{0}$. we get for $u_{i j}^{\frac{r}{r}}(7 a)$

$$
\begin{aligned}
U_{i j} & =U\left(\Omega_{i}^{*}, \Omega_{i}^{r_{0}}, \Omega_{i j}\right) \\
& =\frac{4 m}{5} \sum_{m}\left\{Y_{2, m}\left(\Omega_{i}{ }^{r_{0}}\right)+Y_{2, m}\left(\Omega_{j} \underline{r}_{0}\right)\right\} Y_{2, m}^{*}\left(\Omega_{i j}\right)
\end{aligned}
$$

The transformation of the third part. $Q_{i j}{ }^{ \pm}$, can be performed using several formulas contained in [38]. The result is to be found in [42] :

$$
\begin{aligned}
& Q_{i j}=Q\left(\Omega_{i} \underline{r}_{0}, \Omega_{i} \underline{r}_{0}, R_{i j}\right)
\end{aligned}
$$

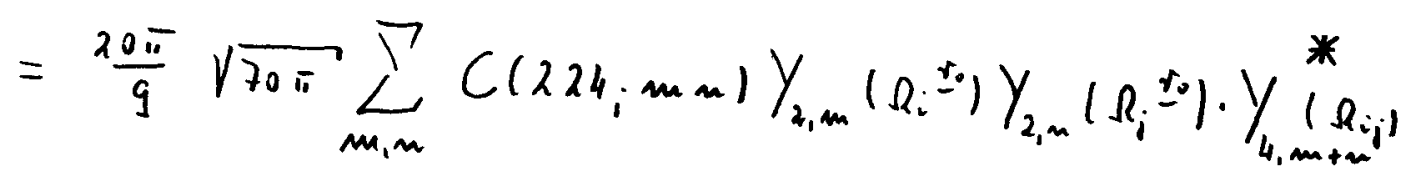

Now we are able to separate the orientational integrations from the translational ones. We have

$$
\begin{aligned}
& f_{i j}^{2} \bar{V}_{i j}=f_{i j}^{2}\left[V_{i j}-\frac{\hbar^{2}}{2 M} \nabla^{2} \ln f_{i j}\right] \\
& =f^{2}\left(r_{i j}\right)\left[V^{0}\left(r_{i j}\right)-\frac{\hbar^{2}}{2 M} \nabla_{t_{i j}}^{2} \ln f\right] \\
& +f^{2}\left(v_{i j}\right) \cdot V^{1}\left(r_{i j}\right) \cdot U\left(\Omega_{i} \stackrel{v}{*}, \Omega_{i} \pm, \Omega_{i j}\right) \\
& +f^{2}\left(v_{i j}\right) \cdot \Gamma\left(r_{i j}\right) \cdot Q\left(\Omega_{i} t_{0}, \Omega_{j} \underline{v}_{0}, \Omega_{i j}\right)
\end{aligned}
$$


$-21-$

For the first part of (15), which does not depend on orientations, we simply get

$$
\begin{aligned}
\left\langle\psi_{i}^{0} \psi_{i}^{0}\left|f^{2} \overline{V^{0}}\right| \psi_{i}^{0} \psi_{i}^{0}\right\rangle & =\left\langle\varphi_{i}^{0} \varphi_{i}^{0}\left|f^{2} \overline{V^{0}}\right| \varphi_{i}^{0} \varphi_{i}^{0}\right\rangle \\
& =\left\langle 00\left|f^{2} \overline{V^{0}}\right| 00\right\rangle
\end{aligned}
$$

Hence orth we use a simple notation:

$|0\rangle$ means $\varphi_{i}^{0}$ or $\gamma_{1,0}(i)$ or $\psi_{i}^{0}$, if the integrand of the matrix element depends on translations alone, or orientations alone, or on both, respectively.

The rest of (15) leads to:

$$
\begin{aligned}
& \left\langle 001 f_{i j}^{2} V_{i j}^{1} u_{i j} \mid 00\right\rangle \\
& =\frac{4 \pi}{5} \sum_{m}\left\{\left\langle 0\left|\gamma_{\lambda, m}\left(\Omega_{i}^{r_{0}}\right)\right| 0\right\rangle+\left\langle 0\left|Y_{\lambda_{1, m}}\right| \Omega_{i} t_{0} \mid 10\right\rangle\right\} . \\
& \cdot\left\langle 001 f_{i j}^{2} V_{i j}^{1} V_{a_{1} m}^{*}\left(\Omega_{i j}\right) \mid 00\right\rangle \\
& \left\langle 00\left|f_{i i}^{2} r_{i j} Q_{i j}\right| 00\right\rangle \\
& =\frac{20 \pi}{9} \sqrt{70 \pi} \sum_{m, m} C(224, \mathrm{~mm}) \cdot\left\langle 001 Y_{2, m}\left(\Omega_{i} \dot{v}_{0}\right) \cdot Y_{2, m}\left(\Omega_{i} \underline{I}_{0}\right) \mid 00\right\rangle \cdot \\
& \cdot\left\langle 00\left|f_{i i}^{2} \Gamma_{i j} Y_{4, m+n}^{*}\left(\Omega_{i j}\right)\right| 00\right\rangle
\end{aligned}
$$

The translational matrix elements can be simplified. With $g(\underline{y})$ representing one of the integrands $f^{2}, f^{2} \bar{v}^{3}, f^{2} V^{1} Y_{2, m}^{*}, f^{2} \Gamma Y_{4, m+n}^{*}$ we have 
$-22-$

$$
\begin{aligned}
& \langle 001 g(\underline{r}) \mid 00\rangle \\
& =\left(\frac{r}{\pi}\right)^{3} \iint e^{-\gamma\left[\left(\underline{R}_{i}-\underline{R}_{i}{ }^{2}\right)^{2}+\left(\underline{R}_{i}-\underline{R}_{i}^{0}\right)^{2}\right]} g(\underline{r}) d^{3} \underline{R}_{i} d^{3} \underline{R}_{i}
\end{aligned}
$$

Dy introducing new coordinates

$$
\begin{array}{ll}
\underline{r}=\underline{r}_{i j}=\underline{R}_{i}-\underline{R}_{i} & \underline{r}_{0}=\underline{R}^{0}-\underline{R}_{i}^{0} \\
\underline{R}=\frac{1}{2}\left(\underline{R}_{i}+\underline{R}_{i}\right) & \underline{R}_{0}=\frac{1}{2}\left(\underline{R}_{j}^{0}+\underline{R}_{i}^{0}\right)
\end{array}
$$

and integrating over $\underline{R}$ we obtain

$$
\langle 00|g| 00\rangle=\left(\frac{\gamma}{2 \pi}\right)^{3 / 2} \int e^{-\frac{\gamma}{2}\left(\underline{x}-\underline{r}_{0}\right)^{2}} g(\underline{r}) d^{3} \pm
$$

Now spherical coordinates are introduced with $\underline{r}_{0}$ as $z$-axis.

$$
\begin{aligned}
& h_{i j}=(\vartheta, \varphi) \\
& \langle 00|g| 00\rangle \\
& =\left(\frac{\gamma}{2 \pi}\right)^{3 / 2} \int_{0}^{\infty} \int_{0}^{\pi} \int_{0}^{2 \pi} e^{-\frac{r}{2}\left(r^{2}+r_{0}^{2}-2 r r_{0} \cos \vartheta\right)} \cdot g(r, \vartheta, \varphi) \cdot r^{2} \sin \vartheta d r d \vartheta d \varphi
\end{aligned}
$$

If $g$ depends only on + (this is the case for $f^{2}$ and $f^{2} \overline{v^{0}}$ ), the angular integrations can easily be carried out:

$$
\langle 00 \mid g(r) 100\rangle=\sqrt{\frac{r}{2 \pi}} \int_{0}^{\infty}\left[e^{-\frac{r}{2}\left(t-r_{0}\right)^{2}}-e^{-\frac{r}{2}\left(r+r_{0}\right)^{2}}\right] g(r) \frac{r}{r_{0}} d r
$$


$-23-$

For $g=f^{2} V^{\wedge} Y_{2, m}^{*} \quad$ and $f^{2} \Gamma Y_{4, m+n}^{*}$ the $\varphi$-intergration shows that all elements with $m \neq 0$ in (17) and with $m+m \neq 0$ in (18) vanish. Thus we obtain

$$
\begin{aligned}
& \left\langle 001 f_{i i}^{2} v_{i j}^{1} u_{i j} \mid 00\right\rangle \\
& =\sqrt{\frac{4_{i m}}{5}}\left\{\left\langle 0\left|\gamma_{2,0}\left(\Omega_{i}-10\right)\right| 0\right\rangle+\left\langle 0\left|Y_{2,0}\left(\Omega_{i} \pm_{0}\right)\right| 0\right\rangle\right\} . \\
& \text { - }\left\langle 00\left|f_{i j}^{2} V_{i j}^{1} \tilde{p}_{2}^{2}\left(v_{i j}\right)\right| 00\right\rangle
\end{aligned}
$$

and

$$
\begin{aligned}
\left\langle 00\left|f_{i j}^{2} \Gamma_{i j} Q_{i j}\right| 00\right\rangle \\
=\frac{10}{3} \pi \sqrt{70} \sum_{\mu} C\left(224_{i} \mu_{i}-\mu\right)\left\langle 00\left|Y_{a_{1} \mu}\left(\Omega_{i}^{\sigma_{0}}\right) \cdot Y_{a_{1}-\mu}\left(Q_{i} \Sigma_{0}\right)\right| 00\right\rangle^{(25)} \\
\cdot\left\langle 00\left|f_{i j}^{2} \Gamma_{i j} \tilde{P}_{4}\left(g_{i j}\right)\right| 00\right\rangle
\end{aligned}
$$

Here $\tilde{P}_{2}$ and $\widetilde{P}_{4}$ are unnormalized Legendre polynomials. Now we see that (24) and (25) can be simplified considerably, if we use (5) and (fa) replacing $\Omega_{i} \pm$ by $\Omega_{i} \underline{\underline{T}}_{0}$ :

$$
\begin{aligned}
& \quad\left\langle 001 f_{i j}^{2} V_{i j}^{1} U_{i j} 100\right\rangle \\
& =\left\langle 001 U\left(R_{i}^{r_{0}}, \Omega_{i} v_{0}\right) 100\right\rangle \cdot\left\langle 001 f^{2}\left(v_{i j}\right) V^{1}\left(v_{i i}\right) \tilde{P}_{2}\left(v_{i j}\right) 100\right\rangle
\end{aligned}
$$

and 


$$
\begin{aligned}
& \left\langle 001 f^{2} \Gamma_{i j} Q_{i j} \mid 00\right\rangle \\
& =\left\langle 00\left|Q\left(\Omega_{i} \underline{r}_{i}, \Omega_{i} \underline{v}^{\prime}\right)\right| 00\right\rangle \cdot\left\langle 00\left|f^{2}\left(v_{i j}\right) \Gamma\left(r_{i j}\right) \tilde{P}_{4}\left(\theta_{i j}\right)\right| 00\right\rangle
\end{aligned}
$$

with $U\left(\Omega_{i} \underline{v}_{i}, \Omega_{i} \underline{v}_{0}\right)=U_{i j} \underline{v}_{0}$ and $Q\left(\Omega_{i} \underline{v}_{0}, \Omega_{i} \underline{v}_{0}\right)=Q_{i j} \underline{v}_{0}$ given by (5) and (7a).

Now the problem is fully separated: The first factor in (26) and in (27) contains only orientational integrations ana can be calculated using only data from the rigid Pa3 lattice.

Nevertheless the calculation is complicated, because the wavefunction $|0\rangle=Y_{1,0}\left(\Omega_{i}\right) ; i \equiv(\underline{m}, g)$, refers to quantization axis $\underline{d}_{g}$ of the sublattice to which the molecule $i$ belongs, whereas $u_{i j} \underline{v}_{0}$ and $Q_{i j}{ }^{{ }^{2}}$ refer to the axis $\underline{r}_{0}=R_{i}^{0}-\underline{R}_{i}^{0}$. Therefore we have to transform again:

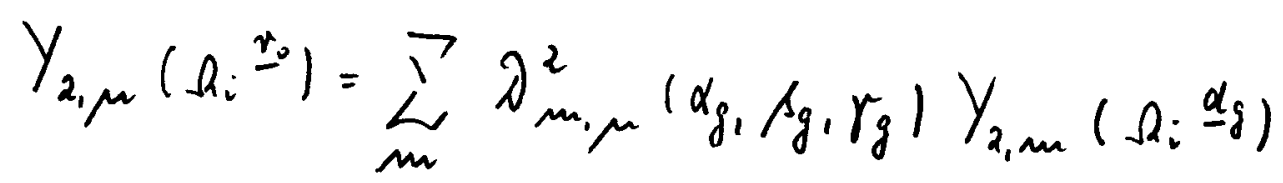

We place this in ${U_{i j}}^{\underline{t}_{0}}$ and ${Q_{i j}}^{\underline{v}_{0}}$ and get after some calculations (appendix XI) quite a simple result:

$$
\begin{aligned}
& \left\langle 00\left|U_{i j} \underline{r}^{-}\right| 00\right\rangle=\frac{2}{5} U\left(\Omega_{g,} \Omega_{h}\right) \\
& \left\langle 00\left|Q_{i j} \underline{r}^{r_{0}}\right| 00\right\rangle=\frac{4}{25} Q\left(\Omega_{g}, \Omega_{h}\right)
\end{aligned}
$$

with $\quad i \equiv(m, g)$ and $j \equiv(\underline{\mu}, h)$ 


$$
\Omega_{g}=h_{\underline{d}_{g}}^{{\underline{x_{0}}}}=\left(\mu_{g}, \alpha_{g}\right) ; \quad \Omega_{h}=h_{\underline{d}_{l}}^{\underline{\tau}_{0}}=\left(\beta_{h}, \alpha_{h}\right)
$$

This result reflects the fact that the Pa3 ground state structure can be obtained by considering arrays of linear quadrupoles in a classical calculation [1]. Quantum mechanics cornes in only by the factors $2 / 5$ in front of $U$ and $(2 / 5)^{2}$ in front of $Q$.

Explicite evaluation of (29) and (30) using (7a) and (5) results in:

$$
\left\langle 00\left|u_{i j}^{r_{0}}\right| 00\right\rangle=\frac{2}{5}\left\{\tilde{P_{2}}\left(\beta_{g}\right)+\tilde{P_{2}}\left(s_{h}\right)\right\}
$$

anc̈

$$
\begin{aligned}
\left\langle 00: Q_{i j}{ }^{\nu_{0}} \mid 00\right\rangle & =\left(3 \cos ^{2} \beta_{g}-1\right) \cdot\left(3 \cos ^{2} \beta_{h}-1\right) \\
& -8 \sin \beta_{g} \cos \beta_{g} \sin \beta_{h} \cos \beta_{h} \cos \left(\alpha_{g}-\alpha_{h}\right) \\
& +\frac{1}{2} \sin ^{2} \beta_{g} \sin ^{2} \beta_{h} \cdot \cos 2\left(\alpha_{g}-\alpha_{h}\right)
\end{aligned}
$$

After this explicite evaluation of the orientational integrations we go back to the translational ones. Starting with (22) it is possible to perform the angular integrations in $\langle 00| f^{2} V^{1} \tilde{p}_{i}\left(l_{i j}|100\rangle\right.$ and $\left\langle 00\left|f^{2} \Gamma \tilde{P}_{4}\left(w_{i j}\right)\right| 00\right\rangle$. But before we do this, we make use of the fact that these two matrix elements and also (23) do not depend on $\underline{r}_{0}$ but only on $r_{0}$. Thus it is useful to split the summation $\nabla^{\prime}$, which occurs in the expression (8) for the ground state ${ }^{i}$ energy, into two parts. First we sum up all molecules $j$, which have the same distance $t_{0}$ to the central molecule $i$ and 
thereby form a shell, and then we sum up all shells.

we obtain

$$
\begin{aligned}
& \sum_{j}^{\prime} \frac{\left\langle f_{i j}^{2} \bar{v}_{i j}\right\rangle}{\left\langle f_{i j}^{2}\right\rangle}=\sum_{S} N_{S} \frac{\left\langle 001 f^{2} \bar{r}^{0} 100\right\rangle}{\left\langle 00 \mid f^{2} 100\right\rangle} \\
& +\sum_{S} \frac{\left\langle 00\left|f^{2} V^{1} \tilde{p}_{2}\right| 00\right\rangle}{\left\langle 00\left|f^{2}\right| 00\right\rangle} \cdot \sum_{j=1}^{N_{S}}\left\langle 00\left|u_{i j}^{t_{0}}\right| 00\right\rangle \\
& +\sum_{S} \frac{\left\langle 00 \mid f^{2} \Gamma \tilde{P}_{4} 100\right\rangle}{\left\langle 001 f^{2} 100\right\rangle} \sum_{j=1}^{N_{s}}\left\langle 00 \mid Q_{i j}^{r_{0}} 100\right\rangle
\end{aligned}
$$

Here $N_{S}$ is the number of the molecules in the shell $S$. The quotients in the sums over $S$ depend on $S$ only by $\gamma_{0}{ }^{(S)}$. For the fcc lattice*:

$$
r_{0}=r_{0}^{(s)}=\sqrt{s} \cdot R_{1}
$$

$R_{1}=$ nearest neighbour separation; $R_{1}$ is a variation parameter and should not be confusea with the fixed experimental value $R_{0}$

The second part in (34) vanishes in the fcc strucutre because of

$$
\sum_{j=1}^{N_{s}}\left\langle 00\left|n_{i j}^{r_{0}}\right| 00\right\rangle=\frac{2}{5} \sum_{j=1}^{N_{s}}\left\{\tilde{P}_{2}\left(s_{j}\right)+\tilde{P}_{\lambda}\left(s_{h} \mid\right\}=0\right.
$$

For all shells. $\beta_{g}$ and $\beta_{h}$ are the angles between the body diagonals $\underline{d}_{j}, \underline{d}_{w}$ and the vector $\underline{r}_{0}=\underline{r}_{i j}^{0}$.

For the hcp lattice we used the $r_{0}^{(s)}$ from the tables in [39]. 
Finally we have to perform the angular integrations in $\left\langle 00\left|f^{2} \Gamma \tilde{p}_{4}\right| 00\right\rangle$. Starting with (22) we obtain

$$
\begin{aligned}
& \langle 00| f^{2} \Gamma \tilde{p}_{4}(00\rangle= \\
& \left(\frac{r}{2 \pi}\right)^{3 / 2} 2 \pi \int_{0}^{\infty} d r r^{2} e^{-\frac{1}{2} r\left(r^{2}+r_{0}^{2}\right)} f^{2}(v) r(v) \cdot \int_{-1}^{+1} e^{r r_{0}+\eta} \tilde{p}_{4}(\eta) d \eta \\
& =\sqrt{\frac{r}{2 \pi}} \int_{0}^{\infty} \frac{t}{r_{0}} f^{2}(v) \Gamma(v) \cdot\left[\left(e^{-\frac{r}{2}\left(r-r_{0}\right)^{2}}-e^{-\frac{r}{2}\left(r+v_{0}\right)^{2}}\right)\left(1+\frac{45}{b^{2}}+\frac{105}{b^{4}}\right)\right. \\
& \left.-\left(e^{-\frac{r}{2}\left(r-v_{0}\right)^{2}}+e^{-\frac{r}{2}\left(r+v_{0}\right)^{2}}\right)\left(\frac{10}{b}+\frac{105}{b^{3}}\right)\right] d r
\end{aligned}
$$

with $b=r r_{0} r$

For nearest neighbours the effective or renormalized $q$-q-coupling constant $\left\langle 001 f^{2} \Gamma \tilde{p}_{4} 100\right\rangle \quad$ is called $r_{0}$ eff. It is different from the rigid lattice $q-q$-coupling constant $\Gamma_{0}$ (III,6) because of the short range correlations between the molecules. For the more distant neighbours the SRC-effect very quickly becomes smaller ana $\left\langle 00\left|f^{2} \Gamma \tilde{p}_{4}\right| 00\right\rangle$ approaches $\Gamma\left(r_{0}\right)$. This can also be seen analytically: For $\gamma r_{0}^{2} \gg 1$ we have $f(r) \equiv 1$ in the whole region, where $\varphi^{0}$ is localized. Therefore

$$
\begin{aligned}
& \left\langle 001 f^{2} \Gamma \tilde{P}_{4} 100\right\rangle=\left\langle 00 \mid \Gamma \tilde{P}_{4} 100\right\rangle \\
& =\left(\frac{r}{\lambda \pi}\right)^{3 / 2} \int e^{-\frac{r}{2}\left(\underline{r}-\underline{r}_{0}\right)^{2}} \Gamma(v) \tilde{P}_{4}(v) d^{3} \underline{v}=\Gamma\left(r_{0}\right)
\end{aligned}
$$


This is equal to $\Gamma\left(r_{0}\right)$, for $\Gamma(r) \cdot \tilde{P}_{4}(v)$ is a solution of the Laplace equation:

$$
\Delta\left(\Gamma(v) \cdot \tilde{P}_{4}(v)\right)=\frac{6 e^{2} q^{2}}{25} \Delta \frac{\tilde{P}_{4}(v)}{r^{5}}=0 \text { for } r \neq 0
$$

Finally we summarize:

$$
\begin{aligned}
& E_{0}^{(2)} / N=\frac{3}{4} \frac{\hbar^{2}}{M} \gamma+\frac{1}{2} \sum_{S} N_{s} \cdot \frac{\left\langle 001 f^{2} \bar{v}_{0} 100\right\rangle}{\left\langle 001 f^{2} 100\right\rangle} \\
& +\frac{1}{2} \sum_{S} \frac{\left\langle 001 f^{2} \Gamma \tilde{P}_{4} 100\right\rangle}{\left\langle 001 f^{2} 100\right\rangle} \sum_{j=1}^{N_{s}}\left\langle 001 Q_{i j} r_{0} \mid 00\right\rangle
\end{aligned}
$$

where $\left\langle 001 f^{2} \bar{v}_{0} 100\right\rangle$ is given by (23)

$$
\begin{aligned}
& \left\langle 001 f^{2} \mid 00\right\rangle \text { is given by (23) } \\
& \left\langle 001 f^{2} \Gamma \tilde{P}_{4} 100\right\rangle \text { is given by (38) }
\end{aligned}
$$$$
<00\left|Q_{i j} \pm_{0}\right| 0_{0}>\text { is given by }
$$

We see that the calculation of all matrix elements has been reduced to the one dimensional radial integrations in (23) and (38). These must be carried out by numerical computation.

\section{c) Numerical procedure}

Instead of using the integration interval $[0, \infty]$, which is the same for all shells, we restrict the numerical integration to the regions where the integranas sufficiently derivate from zero to give contributions within the integration accuracy. The position of these regions depends on the shell number $S$ and their extension is de- 
termined by the width $\wedge / \sqrt{\gamma}$ of the Gaussians in the integrands. One can see that these regions are contained in the following integration intervals:

$$
R_{0}^{(s)}-d \leq r \leq R_{0}^{(s)}+d
$$

with $R_{0}^{(s)}=\sqrt{s} R_{0}$ for the Ecc lattice. $R_{0}^{(s)}$ for the hap lattice is taken from the tables in [39]. If we choose $d$ to be in the order of magnituae of $R_{0}$, it turns out during the variation that this is sufficient for all cases we consider. Actually we choose $d=R_{0}-0.05$ in order to exclude the point $r=0$. The integrands vanish analytically at this point but numerically intermeciate results would become infinite. Of course the intervals (42) can be used only as long as the variation parameter $R_{1}$ remains close to $R_{0}$ during the variation.

Each interval was divided into a large number (e.g. 200) of subintervals, which were integrated by simpson's rule*. Eo ${ }^{*} / N$ was calculated by taking into account the first thirty shells**. For $5>5$ the numerical integration can be avoided by expanding the integrals (23) into a series (appendix $x$ ) or by using (39) when possible.

Division into a smaller number of subintervals, which then are integrated by a higher interpolation rule, keeping the total number of points constant, gives worse results. This probably occurs, because the behaviour of the SRC-function in the region of the hara core prevents a good interpolation of the integrands over larger intervals.

In the hop lattice we must take 61 shells to get the same accuracy. 
The minimum of $E_{0}^{(2)} / N$ with respect to the 6 variation parameters $\mu, R_{1}, k, m, \beta, \gamma_{m}$ was obtained by the method of the steepest decay:

We use a 6-dimensional parameter space. To get a starting point we choose a set of parameters. For this point the gradient is calculated numerically. Then a step into the direction of the negative gradient is done to get a new point, which is closer to the minimum. This procedure is repeated until the coordinates of the minimum are determined with a certain accuracy. 
IV Results of the ground state variation

As a starting point for the variation we have to choose a set of parameters $R_{11}, \gamma, \alpha, K, m, \beta, t_{m}$. For $R_{1}$ we take the experimental value $R_{0} . r$ can be estimated by comparing $1 / \sqrt{r}$ with the mean square displacement of the molecules. This quantity is about 168 of $R_{0}$ for $H_{2}[35 a]$ and about 148 for $D_{2}[36]$.

$\alpha, k, n, \beta, r_{m}$ are chosen such that the shape of the SRC-function $(I I, 21)$

$$
f(r)=e^{-k\left(\frac{\sigma}{r}\right)^{m}}+\alpha e^{-\beta(r-r m)^{2}}
$$

is qualitatively the same as Nosanow's (II,14).

The variation procedure is straight forward (chapter III). It turns out that all parameters except $\alpha$ can be varied freely* and are therefore determined uniquely by minimization of $E_{0}^{(2)}=$ $E_{01}+E_{02}$, which is then a function of $\alpha$ only. The value of $\alpha$ decides whether the cluster expansion $E_{0}=E_{0_{1}}+E_{0_{2}}+E_{0_{3}}+\ldots$ is convergent or not.

* It should be noted that the questions which arise with $\alpha$ can also be discussed using Nosanow's $f$ in its slightly more general form $(I I, 18)$. Here $k^{\prime}$ plays the same role as our $\alpha$. Nosanow's chotce $k^{\prime}=k$ is only justified because of the convergence of the cluster expansion. A very suggestive form results from it: $f(r)=$ $\exp \left[-k V_{0}(r) / 4 \varepsilon\right]$, where $V_{0}$ is the Lennard-Jones potential. 
a) For $\alpha \rightarrow 0$ the function (1) essentially behaves like $\exp \left[-k(\sigma / r)^{m}\right] \quad *$ and does not yield a minimum of $E_{0}^{(2)}$. Thus the cluster expansion does not converge. The physical interpretation is the following: as $K$ is increased the kinetic energy

Eon is continually lowered until the molecules are no longer localized. Numerically a minimum of $E_{0}^{(2)}$ can not be found for $\alpha<0.10$ in the case of $H_{2}$ and for $\alpha<0.07$ in the case of $\partial_{2}$.

b) The larger $\alpha$ is the larger the influence of the maximum in $f(r)$ becomes. If $\alpha$ is chosen too large, the maximum extends to the region $\tau \approx R_{1}$, where the molecule is localized, and destroys a necessary condition $[29,32]$ for $f(r)$, namely

$$
f(r) \approx 1 \quad \text { where }\left|\varphi^{0}(x)\right|^{2} \text { is latge }
$$

It can be shown [37] that this condition is connected with the rate of convergence: The cluster expansion of Nosanow can be written as a power series with respect to a quantity $\delta$, which is defined as

$$
\delta=\left\langle 00\left|f^{2}\right| 00\right\rangle-1 \quad \text { for nearest neighbours }
$$

(For the more distant nelghbours $\delta$ is very close to zero).

Because of the maximum in $f(r) \quad \delta$ obviously increases with Increasing $\alpha$ and the convergence becomes worse. Numerically we obtain a linear dependence between $\delta$ and $\alpha$ [see table 1 ].

To get an estimate of the upper limit for $\alpha$ we perform the variational calculations not only with the function (1) but also with

The function exp $\left[-k(\sigma / r)^{10}\right]$ has already been discussed in [29] for helium. The results are similar to ours. 
Nosanow's SRC-function $(I I, 14)$. In this case we obtain:

\begin{tabular}{l|c|c|c} 
& $\delta$ & & $\delta$ \\
\hline $\mathrm{o}-\mathrm{H}_{2}$ & 0.049 & $\mathrm{p}-\mathrm{D}_{2}$ & 0.070 \\
$\mathrm{p}-\mathrm{H}_{2}$ & 0.052 & $\mathrm{o}-\mathrm{D}_{2}$ & 0.076
\end{tabular}

In the case of helium $\delta$ generally is larger (up to 0.10 ). Nevertheless the convergence of the cluster expansion is fairly good [32] : The leading correction term amounts to 58 of $E_{02}^{v}$ in the worst case. If $E_{03}^{V}$ is included into the variation procedure, the value of the minimum changes only slightly. The same holds for the wave function.

For hydrogen we expect that the convergence is even better, since hydrogen is not as much a quantum crystal as helium is. This can be seen, for example, by comparing the relative mean square displacements of the particles: 168 for $\mathrm{H}_{2}$ and 25-308 for hellum.

Now we go back to our ansatz (1) for the SRC-function. We restrict $\alpha$ so that $\delta$ is always smaller than the minimum value of $\delta$ in (4):

$$
\delta(\alpha)<\delta_{\text {min }}(\text { Nosanow's } f)=0.049
$$

We obtain from table 1:

$$
\alpha<0.14 \quad \text { for } H_{2} \text { and } D_{2}
$$

Table 1 shows the results of the ground state variation. For $0 . H_{2}$ and $p-D_{2}$ the results are given for the whole range of $\alpha$ which has been determined by the considerations in a) and $b$ ). We see that within this range the quantities which can be compared with experimental values change only very slightly, although the change of $\alpha$ is large. In detail the changes are in the case of $0-H_{2}: 308$ for $\alpha, 0.168$ for $R_{1}, 0.558$ for $E_{0}^{(2)} / N, 0.38$ for $\sqrt{\left\langle\underline{1}^{2}\right\rangle}$. For 


\begin{tabular}{|c|c|c|c|c|c|c|c|c|c|c|c|c|c|}
\hline $\begin{array}{l}S C R \\
\text { function }\end{array}$ & crys & $\alpha$ & {$\left[\begin{array}{c}\gamma \\
{\left[A^{-2}\right]}\end{array}\right]$} & K & $\begin{array}{l}R_{1} \\
{[\AA]}\end{array}$ & $M$ & $\begin{array}{c}\beta \\
{\left[\AA^{-2}\right]}\end{array}$ & $\begin{array}{l}r_{m} \\
{[\AA]}\end{array}$ & $\begin{array}{l}E_{0}^{(2)} / N \\
{[\mathrm{mev}]}\end{array}$ & $\mid \begin{array}{l}\frac{\sqrt{\left\langle\underline{m}^{2}\right\rangle}}{R_{1}} \\
{[\%]}\end{array}$ & $\frac{r_{0}^{e f t}}{r_{0}}$ & $f\left(R_{A}\right)$ & $\delta$ \\
\hline \multirow[t]{4}{*}{$\begin{array}{c}x \\
11 \\
0 \\
\\
\frac{x}{4 \pi} \\
\frac{x}{z}\end{array}$} & $0-\mathrm{H}_{2}$ & $\begin{array}{l}0.10 \\
0.11 \\
0.12 \\
0.13\end{array}$ & $\begin{array}{l}4.33 \\
4.43 \\
4.51 \\
4.59\end{array}$ & $\begin{array}{l}0.047 \\
0.040 \\
0.034 \\
0.030\end{array}$ & $\begin{array}{l}3.736 \\
3.738 \\
3.740 \\
3.742\end{array}$ & $\begin{array}{l}15.69 \\
16.28 \\
16.79 \\
17.25\end{array}$ & $\begin{array}{l}6.29 \\
6.10 \\
5.92 \\
5.76\end{array}$ & $\begin{array}{l}3.06 \\
3.05 \\
3.04 \\
3.04\end{array}$ & $\begin{array}{l}-7.27 \\
-7.28 \\
-7.30 \\
-7.31\end{array}$ & $\begin{array}{l}15.1 \\
15.1 \\
15.1 \\
15.0\end{array}$ & $\begin{array}{l}0.987 \\
0.997 \\
1.005 \\
1.012\end{array}$ & $\begin{array}{l}1.004 \\
1.005 \\
1.006 \\
1.007\end{array}$ & $\begin{array}{l}0.027 \\
0.034 \\
0.041 \\
0.046\end{array}$ \\
\hline & $p-D_{2}(f c c)$ & $\begin{array}{l}0.07 \\
0.09 \\
0.11 \\
0.13\end{array}$ & $\begin{array}{l}8.12 \\
8.38 \\
8.60 \\
8.79\end{array}$ & $\begin{array}{l}0.027 \\
0.018 \\
0.013 \\
0.009\end{array}$ & $\begin{array}{l}3.519 \\
3.523 \\
3.526 \\
3.529\end{array}$ & $\begin{array}{l}19.91 \\
21.47 \\
12.76 \\
23.92\end{array}$ & $\begin{array}{c}12.40 \\
11.31 \\
10.47 \\
9.80\end{array}$ & $\begin{array}{l}2.99 \\
2.98 \\
2.97 \\
2.96\end{array}$ & $\begin{array}{l}-12.34 \\
-12.37 \\
-12.39 \\
-12.41 \\
\end{array}$ & $\begin{array}{l}11.8 \\
11.7 \\
11.6 \\
11.5\end{array}$ & $\begin{array}{l}0.998 \\
1.008 \\
1.018 \\
1.025\end{array}$ & $\begin{array}{l}1.002 \\
1.003 \\
1.004 \\
1.006 \\
\end{array}$ & $\begin{array}{l}0.017 \\
0.028 \\
0.038 \\
0.047 \\
\end{array}$ \\
\hline & $\begin{array}{l}\mathrm{p}-\mathrm{H}_{2}(\mathrm{hcp}) \\
\mathrm{p}-\mathrm{H}_{2} \text { (fcc) }\end{array}$ & $\begin{array}{l}0.10 \\
0.10\end{array}$ & $\begin{array}{l}3.82 \\
3.83\end{array}$ & $\begin{array}{l}0.083 \\
0.083\end{array}$ & $\begin{array}{l}3.764 \\
3.764\end{array}$ & $\begin{array}{l}13.61 \\
13.65\end{array}$ & $\begin{array}{l}5.75 \\
5.76\end{array}$ & $\begin{array}{l}3.08 \\
3.08\end{array}$ & $\begin{array}{l}-6.69 \\
-6.68 \\
\end{array}$ & $\begin{array}{l}15.7 \\
15.7\end{array}$ & - & $\begin{array}{l}1.004 \\
1.004\end{array}$ & $\begin{array}{l}0.018 \\
0.018 \\
\end{array}$ \\
\hline & $\begin{array}{l}o-D_{2}(h c p) \\
0-D_{2}(f c c) \\
\end{array}$ & $\begin{array}{l}0.09 \\
0.09\end{array}$ & $\begin{array}{l}7.93 \\
7.93 \\
\end{array}$ & $\begin{array}{l}0.025 \\
0.025\end{array}$ & $\begin{array}{l}3.543 \\
3.543\end{array}$ & $\begin{array}{l}20.27 \\
20.28 \\
\end{array}$ & $\begin{array}{l}10.91 \\
10.91\end{array}$ & $\begin{array}{l}3.00 \\
3.00\end{array}$ & $\begin{array}{l}-11.56 \\
-11.56 \\
\end{array}$ & $\begin{array}{l}12.1 \\
12.1\end{array} \mid$ & - & $\begin{array}{l}1.004 \\
1.004\end{array}$ & $\begin{array}{l}0.027 \\
0.027 \\
\end{array}$ \\
\hline \multirow{2}{*}{ 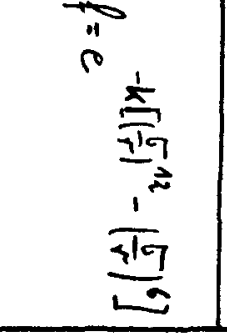 } & $\begin{array}{l}o-H_{2}(f c c) \\
p-D_{2}(f c c) \\
\end{array}$ & - & $\begin{array}{l}3.78 \\
7.04\end{array}$ & $\begin{array}{l}0.204 \\
0.217\end{array}$ & $\begin{array}{l}3.728 \\
3.513 \\
\end{array}$ & - & - & - & $\begin{array}{r}-7.17 \\
-12.26 \\
\end{array}$ & $\begin{array}{l}15.3 \\
11.9 \\
\end{array}$ & $\begin{array}{l}0.939 \\
0.965\end{array}$ & $\begin{array}{l}1.036 \\
1.050 \\
\end{array}$ & $\begin{array}{l}0.049 \\
0.070 \\
\end{array}$ \\
\hline & $\begin{array}{l}\mathrm{p}-\mathrm{H}_{2}(\mathrm{fcc}) \\
\mathrm{o}-\mathrm{D}_{2}(\mathrm{fcc})\end{array}$ & - & $\begin{array}{l}3.54 \\
6.65\end{array}$ & $\begin{array}{l}0.220 \\
0.238\end{array}$ & $\begin{array}{l}3.759 \\
3.534\end{array}$ & - & - & - & $\begin{array}{r}-6.60 \\
-11.48 \\
\end{array}$ & $\begin{array}{l}15.7 \\
12.2\end{array}$ & - & $\begin{array}{l}1.040 \\
1.050\end{array}$ & $\begin{array}{l}0.052 \\
0.076 \\
\end{array}$ \\
\hline
\end{tabular}


deuterium the situation is even better.

Most of the parameters contained in the sRC-function $f(r)$ change rather rapidly with a change of $\alpha$ when the variation is performed, but in such a manner that the shape of $f(T)$ is not essentially altered. (For example the changes of $k$ and $n$ compensate each other up to a certain extent). This emphasizes the fact that the parameters in $f(r)$ are pure variational parameters and cannot be compared with physical ones. Only the shape of $f(r)$ is relevant for the problem.

Though the parameter $\alpha$ could not be determined by variation, we can now conclude that the variational problem $(I I, 41)$ has been solved by giving stable results within about 18.

Actually it does not matter which value of $\alpha$ we select from the region of convergence given by a) and b). Therefore in table 1 only 1 value of $\alpha$ is chosen for $p-H_{2}$ (hcp) and $0-\lambda_{2}$ (hcp) as well as for the hypothetic $p-H_{2}$ (fcc) and $0-\partial_{2}$ (fcc). Since the differences between the results for the hcp and fcc lattice are very small the control calculations with Nosanow's $f$ have oeen made only for the fcc lattice.

To give an illustration of the numerical results in table 1 , the SRC-function $f(r)$, the one-particle-function $\varphi \circ(r)$, and the effective potential $V_{0} f f(r)$ are shown in the figures 1 and 2 for $\mathrm{O}-\mathrm{H}_{2}$ and $\mathrm{p}-\lambda_{2}$ respectively. Nosanow's $f$ and his results are also shown for comparison.

We see that our ansatz (1) for $f(r)$ fulfils the condition $f \rightarrow 1$ where $\varphi_{0}^{2}$ is large, better than Nosanow's $f(r)$ does. The difference is even better seen at the effective potential, which should not differ from $V_{0}$ for large values of $r$, because the short range correlations can play a role only inside and near the hard core.

Let us now compare the results of the ground state variation with 
the experimental results, which are given in table 2.

1) The nearest neighbour separation $R_{0}$ is well approximated. Nevertheless a slight discrepancy can be seen when we compare for example $0-H_{2}$ (fcc) and $p-H_{2}$ (hcp). The observed difference in $R_{0}$ is smaller than the calculated one. One reason is that the q-q-forces, which are attractive in the average, are overemphasized by the bad value of $r_{0}$ eff. See 2 ).

\section{Table 2 Experimental results}

\begin{tabular}{|c|c|c|c|c|c|}
\hline crystal & $\begin{array}{c}R_{0}[R] \\
x-\text { ray neu } \\
\text { diffractio }\end{array}$ & atron & $\begin{array}{l}E_{\mathrm{o}} / \mathrm{N} \\
{[\mathrm{meV}]}\end{array}$ & $\begin{array}{c}\frac{\sqrt{\left\langle\underline{m}^{2}\right\rangle}}{R_{0}} \\
{[\%]}\end{array}$ & $\frac{\Gamma_{0}^{\text {eff }}}{\Gamma_{0}}$ \\
\hline $\begin{array}{l}o-\mathrm{H}_{2}(f c c) \\
\mathrm{p}-\mathrm{H}_{2}(h c p)\end{array}$ & $\begin{array}{r}3.756 \pm 0.01 \\
3.761 \pm 0.007 \\
{[7]}\end{array}$ & & $\begin{array}{c}-7.94 \\
\text { see }[31]\end{array}$ & $\begin{array}{l}15.7 \\
{[35 a]}\end{array}$ & $\begin{array}{c}0.67-0.82 \\
- \\
\text { see }[22]\end{array}$ \\
\hline $\begin{array}{l}p-D_{2}(f c c) \\
o-D_{2}(h c p)\end{array}$ & $\begin{array}{r}3.59 \pm 0.01 \\
3.600 \pm 0.04 \\
{[8]}\end{array}$ & $\begin{array}{r}3.588 \pm 0.01 \\
3.59 \\
{[11]}\end{array}$ & $\begin{array}{l}-11.89 \\
\operatorname{see}[31]\end{array}$ & $\begin{array}{r}13.9 \\
{[46]}\end{array}$ & $\begin{array}{c}0.79-0.88 \\
- \\
\text { see }[22]\end{array}$ \\
\hline
\end{tabular}

On the other hand the differences between $R_{0}$ and $R_{1}$ are smaller than the changes which come in by the uncertainties of the Lennard-Jones parameters $\sigma$ and $\varepsilon$. If we take $\sigma=2.59 \AA, \varepsilon=$ $36.7 \mathrm{~K}$ from [43] Instead of our $\sigma=2.93 \dot{A}, \varepsilon=37 \mathrm{~K}$, we get $R_{1}=$ $3.769 \dot{A}$ instead of $3.736 \AA$ for $0 . H_{2}$ with $\alpha=0.10$.

2) $\Gamma_{0}$ eff $/ \Gamma_{0}$ has been determined by a lot of experiments, such as nuclear magnetic resonance (NMR), neutron and Raman scattering, see [22]. The results lie between about 0.7 and 0.9 , whereas our calculation essentially yields 1.0. Harris [23] obtained 0.84 with 
more sophisticated methods: The value 0.84 is composed of the factors 0.94 from "static phonon renormalization", 094 from "dynamic phonon renormalization", and 0.95 from dielectric screening. The "static renormalization" is calculated by using Nosanow's SRC-function and data from $p-H_{2}$; the anisotropic $q-q-$ potential between the $\mathrm{O}-\mathrm{H}_{2}$ molecules then is averaged over the zero point motion. Thus the factor 0.94 corresponds to our result 0.939 (table 1), obtained by Nosanow's function. The "dynamic renormalization" results from the perturbation of the orientational order of the molecules due to the phonons. The effect has been calculated by Harris with perturbation theory using a Debye spectrum for the phonons.

3) Similar to the ground state energy the mean square displacement must also be calculated by a cluster expansion [45] :

$$
\left\langle\underline{u}_{i}^{2}\right\rangle=\left\langle 0\left|\underline{u}_{i}^{2}\right| 0\right\rangle+\sum_{j}^{\prime}\left\{\frac{\left\langle 00\left|\underline{u}_{i}^{2} f_{i j}^{2}\right| 00\right\rangle}{\left\langle 001 f_{i j}^{2} \mid 00\right\rangle}-\left\langle 0\left|\underline{u}_{i}^{2}\right| 0\right\rangle\right\}
$$

$\left\langle 0\left|\underline{u}_{i}^{2}\right| 0\right\rangle$ is equal to $3 /(2 \gamma \mid)\left\langle 00\left|\underline{u}_{i}{ }^{2} f_{i j}{ }^{2}\right| 00\right\rangle$ is calculated by the methods described in chapter VII and yields $3 / 2 r \cdot<001 f^{2} i 100>\cdot \eta$. Thus we get

$$
\left\langle\underline{\underline{u}}_{i}^{2}\right\rangle=\frac{3}{2 r}\left[1+\sum_{s=1} N_{s}\left(\eta_{s}-1\right)\right]
$$

$N_{\mathrm{s}}$ is the number of molecules in the shells.

For nearest neighbours $\eta$ turns out to be between 0.9855 and 0.9976 for the different cases we consider. For more distant neighbours $\eta$ is very close to unity. Thus the value $3 / 2 \gamma$ is reduced by a factor $0.826-0.971$ due to the short range correlations. In table 1 the quantity $\sqrt{\left\langle\underline{\underline{\mu}}^{2}\right\rangle} / R_{1}$ is listed.

Schott $[35 a]$ has measured $\sqrt{\left\langle\underline{u}^{2}\right\rangle}=0.59 \AA=15.78$ of $R_{0}$ for 
hydrogen containing $6880-\mathrm{H}_{2}$. The experiment was performed at $4.3^{\circ} \mathrm{K}$ with the disordered hcp phase. Thus we must compare it with our value 15.78 for $p-H_{\lambda}$ (hcp). Of course this exact agreement occurs by chance. A similar comparison can be made between our value 12.18 for $O-D_{\lambda}(\mathrm{hcp})$ and the experimental result 13.98 for $0-D_{2}$ at $5^{\circ} \mathrm{K}$.

4) We still have to discuss the most important quantity: the ground state energy $E_{0}$. Unfortunately $E_{0}$ has not yet been measured for the ordered state, but only for the hop lattice. We obtain a good agreement only for deuterium: - $11.56 \mathrm{meV}$ compared with $-11.89 \mathrm{meV}$. For hydrogen the calculated $E_{0}^{(2)} / \mathrm{N}=$ $-6.69 \mathrm{meV}$ still lies $1.25 \mathrm{meV}$ above the experimental $E_{0} / \mathrm{N}=$ $-7.94 \mathrm{meV}$. Inclusion of $E_{03}^{V}$ into the variation will certainly not change this discrepancy very much, as was already discussed in [32].

Another question is whether a further refinement of the SRCfunction will improve the situation. This seems improbable looking at the small improvement of $0.1 \mathrm{meV}$, which we have reached by our $f(r)$ compared with Nosanow's.

Thus it is obvious that the modified Hartree ansatz $(I, 1)$ must be criticized. Here each molecule moves in the mean field which is produced by the surrounding molecules. But the motion is independent from the motion of the other molecules, whereas in reality there is a correlation. This long range correlation is not contained in $E_{0}=\left(\phi_{0}, H \phi_{0}\right) /\left(\phi_{0}, \phi_{0}\right)$ aft least for a part of the discussed discrepancy responsible? as the Debye temperature of hydrogen $110^{\circ} \mathrm{K}=10 \mathrm{meV}[35 \mathrm{a}, 44]$ is larger than the ground state energy per molecule.

The long range correlations will be calculated in part $c$ of this work using a time dependent Hartree method. 


\section{Collective excitations}

V Random Phase Approximation (RPA)

We want to develop a theory which describes all low-lying collective excitations (phonons, librons, and "mixed" excitations, which arise because of the coupling of the two) in pure $0-H_{2}$ and $p-\partial_{2}$. We shall work within the frame of the RPA or time dependent Hartree method at $T=0$. This means that the damping of the exclations will be neglected.

The theory will first be developed neglecting short range correlations (SRC). In the next chapter we shall see which changes come in by the SRC.

We start as we did in chapter I: The ordered ground state is approximately described by the Hartree wave function

$$
\phi=\frac{N}{\|_{i}} \psi_{i}^{0}
$$

with

$$
\begin{aligned}
& \psi_{i}^{0}=\varphi^{0}\left(\underline{R}_{i}-\underline{R}_{i}^{0}\right) \cdot Y_{1,0}\left(\Omega_{i}\right) \\
& i \equiv(\underline{m} \cdot g)
\end{aligned}
$$

There are two types of single particle excitations; three translational ones

$$
\psi_{i}^{k}=\varphi^{k}\left(\underline{R}_{i}-\underline{R}_{i}^{0}\right) \cdot Y_{1,0}\left(\Omega_{i}\right) ; k=x_{1} y_{1} z
$$

and two orientational ones 
$-40-$

$$
\psi_{i}^{\alpha}=\varphi^{0}\left(\underline{R}_{i}-\underline{R}_{i}^{0}\right) \cdot Y_{1, \alpha}\left(\Omega_{i}\right) ; \alpha=+1_{1}-1
$$

For both types we use the shorthand

$$
A=k_{1} \alpha=x_{1} y_{1} z_{1}+1,-1
$$

A labels 5 single particle excitations.

Since there are 4 molecules per unit cell in the Pas ground state structure, we expect an eigenvalue problem of dimension 20.

We define operators $b_{i}^{\mu+}, b_{i}^{\mu}$, which create and destruct a molecule in the state $\mu$ with the wavefunction $\psi_{i}^{\mu}$. The index $\mu$ labels ground and excited states of the molecule:

$$
\mu=0, A=0, x_{1}, y_{1},+1,-1
$$

(1) can be rewritten then

$$
\left.|0\rangle=\left|\begin{array}{llll}
0 & 0 \\
1 & 2
\end{array} \cdots i_{i}^{0} \cdots, \frac{0}{N}\right\rangle=\prod_{i} b_{i}^{0+1}\right\rangle
$$

where $\mid>$ is the vacuum state.

The localized sing le particle excitations are written:

$$
\left.\left|\begin{array}{l}
A \\
j
\end{array}\right\rangle=\mid \begin{array}{lllll}
0 & 0 & \ldots & A \\
1 & 2 & \cdots & 0 \\
j
\end{array}\right]=b_{j}^{A+} b_{i}^{0}|0\rangle
$$


In order to get the energy of this excitation we use the Hartree equation

$$
T_{i}^{\mu \nu}+\sum_{j}^{\nabla^{\prime}}\left\langle\mu o\left|W_{i j}\right| v o\right\rangle=E_{i}^{\mu \nu}
$$

with $W_{i j}=$ intermolecular interaction $i \underset{j}{\sum_{j}^{\prime}}=\sum_{j(\neq i)}$

$$
T_{i}^{\mu \nu} \quad=\text { kinetice energy }
$$

$\Sigma^{\prime}\left\langle\mu \nu\left|w_{i j}\right| \nu 0\right\rangle=$ molecular field on site $i$

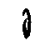

We choose coordinate systems so that ( 8 ) becomes diagonal (these coordinates may be different for different sublattices):

$$
T_{i}^{\mu v}+\sum_{i}^{\prime}\left\langle\mu 0\left|W_{i j}\right| v_{0}\right\rangle=E^{\mu} \delta^{\mu v}
$$

Then

$$
\varepsilon_{A}=E^{A}-E^{0}
$$

is the single particle excitation energy in the Hartree approximation. $\varepsilon_{A}$ is independent of $i$.

After this static calculations we go on to the dynamics of the problem.

We consider the motion of a molecule $i$ in a time dependent molecular field which describes the effect of all the other molecules on the molecule 1 , whereby the state of the whole crystal is given by a time dependent density matrix. This method has been 
developed by Brenig [33] for the case of phonons; we generalize 1t for our case.

The density matrix is defined as

$$
\rho_{i j}^{\mu v}(t)=\left\langle b_{i}^{\nu+}(t) b_{i}^{\mu}(t)\right\rangle
$$

If the expectation value is taken with respect to the Hartree ground state, we simply get

$$
\begin{aligned}
\rho_{i j \text { Ha+t+ee }}^{\mu v} & =\left\langle 0\left|b_{i}^{v+} b_{i} \mu\right| 0\right\rangle \\
& =N_{i}^{\mu} \delta_{i i}^{\mu v}
\end{aligned}
$$

with $N_{i}{ }^{\mu}= \begin{cases}1 & \text { for } \mu=0 \\ 0 & \text { for } \mu=A\end{cases}$

In an ideal crystal we have

$$
\rho_{i j}^{\mu v}=\rho_{i}^{\mu v} \delta_{i j}
$$

which means that every lattice site is occupied by just one molecule.

We must solve the equation of motion

$$
\begin{aligned}
i \dot{\rho}_{i}^{\mu v} & =[H, \rho]]_{i}^{\mu v} \quad(\hbar=1) \\
& =[T, \rho]]_{i}^{\mu v}+[v, \rho]{ }_{i}^{\mu v}
\end{aligned}
$$


Here $V_{i}$ is the above mentioned time dependent molecular field

$$
V_{i}^{\mu \nu}=\sum_{j, \eta, \xi}^{\prime}\left\langle\mu \xi\left|W_{i j}\right| \nu \eta\right\rangle \rho_{j} \eta \xi
$$

If we insert $\rho_{\text {Hartree }}$ from (12) we once more get the time independent molecular field

$$
\begin{aligned}
& V_{i \text { Hartree }}^{\mu r}=\sum_{j, \xi, \tau}^{\prime}\left\langle\mu \xi\left|\omega_{i j}\right| \nu \eta\right\rangle \rho_{j}^{\rho} \rho_{\text {Hartree }}^{\eta \xi} \\
& =\sum_{i}^{\prime}\left\langle\mu_{0}\left|w_{i j}\right| v_{0}\right\rangle=\delta \mu v \sum_{i}^{\prime}\left\langle\mu_{0}\left|W_{i j}\right| \mu_{0}\right\rangle
\end{aligned}
$$

The equation of motion explicitely reads

$$
i \dot{\rho}_{i}^{\mu v}=\left(T_{i}^{\mu \mu}-T_{i}^{\nu \nu}\right) \rho_{i}^{\mu v}+
$$

$$
\sum_{\zeta, 5, i} \sum_{j}^{\prime}\left[\left\langle\mu \xi\left|W_{i j}\right| 5 \eta\right\rangle \rho_{i}^{\eta \xi} \rho_{i}^{5 \nu}-\left\langle 5 \xi\left|W_{i j}\right| \nu_{\eta}\right\rangle \rho_{j}^{\eta \xi} \rho_{i}^{\mu \xi}\right]
$$

Now we consider small admixtures of excited states to the Hartree ground state and linearize $\rho$ : 


$$
\begin{aligned}
\rho_{i}^{\mu \nu} & =\rho_{i \text { Hartice }}^{\mu \nu}+\sigma_{i}^{\mu \nu} e^{-i \omega t}+\sigma_{i}^{\nu \mu^{*}} e^{i \omega t} \\
& =N_{i}^{\mu \nu} \delta^{\mu \nu}+\left(\sigma_{i}^{\mu \nu} e^{-i \omega t}+\text { h.c. }\right)
\end{aligned}
$$

With this $\rho$ we linearize the equation of motion (19). We use the Hartree equation (9) and get after some calculation

$$
\begin{aligned}
& \omega\left(\sigma_{i}^{\mu \nu} e^{-i \omega t}-\text { h.c. }\right)=\left(E_{i}^{\mu}-E_{i}^{\nu}\right)\left(\sigma_{i}^{\mu v} e^{-i \omega t}+\text { h.c. }\right) \\
& \left.+\left(N_{i}^{\nu}-N_{i}^{\mu}\right) \sum_{j, \xi_{i \eta}}^{\prime}\left\langle\mu \xi\left|W_{i j}\right| v \eta\right\rangle \mid \sigma_{j}^{\eta \xi} e^{-i \omega t}+\text { h.c. }\right)
\end{aligned}
$$

From this equation we get

$$
\left[\omega-\left(E_{i}^{\mu}-E_{i}^{\nu}\right)\right] \Gamma_{i}^{\mu \nu}=\mid N_{i}^{\nu}-N_{i}^{\mu} \sum_{i, \mu, \xi}\left\langle\mu \xi\left|W_{i j}\right| \nu \eta\right\rangle \sigma_{i} \eta \xi
$$

Using (13) we see that only the quantities $\sigma_{i}^{A 0}$ and $\sigma_{i}^{A A}$ are different from zero. Thus we obtain two equations from (22):

$$
\begin{aligned}
\left(\omega-\varepsilon_{A} \mid \sigma_{i}^{A 0}\right. & =\sum_{j, B}^{\prime}\left[\left\langle A 0\left|W_{i j}\right| 0 B\right\rangle \sigma_{j}^{B 0}+\left\langle A B\left|W_{i j}\right| 00\right\rangle \sigma_{j}^{0 B}\right] \\
-\left(\omega+\varepsilon_{A}\right) \sigma_{i}^{0 A} & =\sum_{j . B}^{1}\left[\left\langle A B\left|W_{i j}\right| 00\right\rangle^{*} \sigma_{j}^{B 0}+\left\langle A 0\left|W_{i j}\right| 0 B\right\rangle^{*} \sigma_{j}^{0 B}\right]
\end{aligned}
$$


$-45-$

In order to solve these RPA-equations we must express the matrix elements $\left\langle A B\left|W_{i j}\right| 00\right\rangle$ by the elements $\left\langle A 0\left|W_{i j}\right| 0 B\right\rangle$. We can choose the functions $\varphi^{0}\left(\underline{R}_{i}-{\underline{R_{i}}}^{\circ}\right)$ and $\varphi^{k}\left(\underline{R}_{i}-\underline{R}_{i}^{\circ}\right)$ real. For the spherical harmonics $Y_{1, m}\left(\Omega_{i}\right)$ we use the sign convention of condor and shortly, namely $Y_{1, \alpha}{ }^{*}=-Y_{1,-\alpha}$. Then we obtain the following relations

$$
\begin{aligned}
& \left\langle k L\left|W_{i j}\right| 00\right\rangle=\left\langle k 0\left|W_{i j}\right| 0 L\right\rangle=\left\langle k 0\left|W_{i j}\right| 0 L\right\rangle^{*} \\
& \left\langle\alpha k\left|W_{i j}\right| 00\right\rangle=\left\langle\alpha 0\left|W_{i j}\right| 0 k\right\rangle=-\left\langle\bar{\alpha} 0\left|W_{i j}\right| 0 k\right\rangle^{*} \\
& \left\langle k \alpha\left|W_{i j}\right| 00\right\rangle=-\left\langle k 0\left|W_{i j}\right| 0 \bar{\alpha}\right\rangle=\left\langle k 0\left|W_{i j}\right| 0 \alpha\right\rangle^{*} \\
& \left\langle\alpha \alpha\left|W_{i j}\right| 00\right\rangle=-\left\langle\alpha 0\left|W_{i j}\right| 0 \bar{\alpha}\right\rangle=-\left\langle\bar{\alpha} 0\left|W_{i j}\right| 0 \alpha\right\rangle^{*} \\
& \left\langle\alpha \bar{\alpha}\left|W_{i j}\right| 00\right\rangle=-\left\langle\alpha 0\left|W_{i j}\right| 0 \alpha\right\rangle=-\left\langle\bar{\alpha} 0\left|W_{i j}\right| 0 \bar{\alpha}\right\rangle^{*}
\end{aligned}
$$

with $K, L=x_{1}, y, z$

$$
\alpha=+1,-1 \quad ; \bar{\alpha}=-\alpha
$$

Since $|\alpha\rangle^{*}$ must be expressed by $|\bar{\alpha}\rangle$, we cannot use our shorthand notation $A=k, \alpha$ for the next steps. First we consider the RPA-equations (23) for the case $A=K$. With the aid of the relations (24) the two equations (23) can be combined to give

$$
\begin{aligned}
& \frac{\omega^{2}-\varepsilon_{k}^{2}}{2 \varepsilon_{k}}\left(\sigma_{i}^{k 0}+\sigma_{i}^{0 k}\right)= \\
& \sum_{j}^{\prime}\left\{\sum_{L}\left\langle k 0\left|W_{i j}\right| 0 L\right\rangle\left(\sigma_{j}^{L 0}+\sigma_{j}^{0 L}\right)+\sum_{\Lambda}\left\langle k 0\left|\omega_{i j}\right| 0 \rho\right\rangle\left(\sigma_{i} \Delta 0-\sigma_{j} 0 \bar{s}\right)\right\} \\
& \text { with } s=+1,-1
\end{aligned}
$$


Similarly we obtain for the case $A=\alpha$ :

$$
\begin{aligned}
& \frac{\omega^{2}-\varepsilon_{\alpha}^{2}}{2 \varepsilon_{\alpha}}\left(\sigma_{i}^{\alpha 0}-\sigma_{i}^{0 \bar{\alpha}}\right)= \\
& \sum_{j}^{\prime}\left\{\sum_{L}\left\langle\alpha 0\left|W_{i j}\right| 0 L\right\rangle\left(\sigma_{j}^{L 0}+\sigma_{j}^{0 L}\right)+\sum_{\beta}\left\langle\alpha 0\left|w_{i j}\right| 0 \beta\right\rangle\left(\sigma_{i}{ }^{\mu 0}-\sigma_{j}^{0 \bar{\beta}}\right)\right\}
\end{aligned}
$$

Apart from (24) we have used the fact that the two orientational single particle excitations (3) are degenerate:

$$
\varepsilon_{\alpha}=\varepsilon_{\bar{\alpha}}
$$

This is easily shown by

$$
\begin{aligned}
\varepsilon_{\alpha} & =T_{i}^{\alpha \alpha}-T_{i}^{00}+\sum_{i}^{\prime}\left[\left\langle\alpha 0\left|w_{i j}\right| \alpha 0\right\rangle-\left\langle 00\left|W_{i j}\right| 00\right\rangle\right] \\
& =\sum_{i}^{\prime}\left[\left\langle\bar{\alpha}_{0}\left|w_{i}\right| \bar{\alpha} 0\right\rangle-\left\langle 00\left|w_{i j}\right| 00\right\rangle\right]=\varepsilon_{\bar{\alpha}}
\end{aligned}
$$

with $T_{i}^{\alpha \alpha}=\bar{T}_{i}^{\bar{\alpha} \bar{\alpha}}=\bar{T}_{i}^{00}$, because the orientational excthtions do not change the rotational energy.

Now we make use of the translational invariance of the lattice by an ansatz in the form of plane waves:

$$
\begin{aligned}
& \sigma_{i}^{k_{0}}+\sigma_{i}^{0 k}=\tilde{C}_{g}^{k} e^{i \underline{k} \underline{R}_{i}^{0}} \\
& \sigma_{i}^{\alpha_{0}}-\sigma_{i}^{0 \bar{\alpha}}=\tilde{C}_{g}^{\alpha} e^{i \xi \underline{R}_{i}^{0}}
\end{aligned}
$$


Here the components $\tilde{C}_{j}^{A}$ of the polarisation vector must carry the index $g$, because of $i \equiv(m, g)$.

Substituting the ansatz (19) into the equations (25) and (26) the order of our eigenvalue problem is reduced from $20 \cdot N$ to 20. We can again use our shorthand notation $A=k, \alpha$ respectively $B=L, s$ and we obtain

$$
\frac{\omega^{2}-\varepsilon_{A}^{2}}{2 \varepsilon_{A}} \tilde{C}_{j}^{A}=\sum_{B} \sum_{j}^{\prime} e^{i \underline{h}_{i j}^{0}}\left\langle A 0\left|W_{i j}\right| 0 B\right\rangle \tilde{C}_{h}^{B}
$$

The summation overjmay be split: $j \equiv(\underline{\mu}, h)$

$$
\frac{\omega^{2}-\varepsilon_{A}^{2}}{2 \varepsilon_{A}} \tilde{C}_{g}^{A}=\sum_{B_{1} h} \tilde{G}_{g h}^{A B} \tilde{C}_{h}^{B}
$$

with

$$
\tilde{G}_{g h}^{A B}=\sum_{\underline{\mu}}^{\prime} e^{i \underline{k} \underline{x}_{i i}^{0}}\left\langle A 0\left|W_{i j}\right| O B\right\rangle
$$

Here $\Sigma^{\prime}$ denotes the summation over all molecules of the sublattice $\tilde{h}$ for $h \neq g$ and the summation over all molecules without $\underline{\mu}=$ 쓰 for $h=g$.

For the explicite solution of the eigenvalue problem (31) we must remember that we have used different coordinate systems in the different sublattices so that the matrix of the single particle excitation energies is diagonal. [ See equations (8) and (9) ] . On the other hand the wave vector $\underline{k}$ should be expressed in a crystal-fixed frame as usual. 
Therefore we transform the different sublattice systems to a coordinate system whose axes are parallel to those of the cubic lattice.

First we consider the subspace of the tranlational exciations. Here the transformation is performed by a rotation of the coordinate systems in ordinary 3-dimensional space:

$$
\begin{aligned}
& \tilde{C}_{g}^{k}=\sum_{L} R^{k L}\left(\alpha_{g}, s_{g}, \gamma_{g}\right) C_{g}^{L} \\
& \tilde{C}_{g}^{k}=\sum_{L} R_{g}^{k L} C_{g}^{L}
\end{aligned}
$$

with $\alpha_{1} \wedge, \gamma=$ Euler angles of the rotation.

Within the subspace of the orientational exctations the matrix of the excitation energies is always diagonal, because it is propartional to the unit matrix $\left(\varepsilon_{\alpha}=\varepsilon_{\bar{\alpha}}\right)$. Therefore we can choose

$$
\tilde{C}_{g}^{\alpha}=c_{g}^{\alpha}
$$

(33) and (34) may be combined:

$$
\tilde{C}_{g}^{A}=\sum_{B} R_{g}^{A B} C_{g}^{B}
$$

with

$$
R_{g}^{\alpha \alpha}=1 \quad ; \quad R_{g}^{\alpha \bar{\alpha}}=0
$$


and

$$
R_{g}^{k \alpha}=R_{g}^{\alpha k}=0
$$

because we do not want to have a mixed representation for the translational and orientational exctations.

By (35) the eigenvalue equation (31) is transformed to

$$
\omega^{2} \sum_{B}\left(\varepsilon_{g}^{-1}\right)^{A B} C_{g}^{B}=\sum_{B} \varepsilon_{g}^{A B} C_{g}^{B}+2 \sum_{B, L} G_{g h}^{A B} C_{h}^{B}
$$

with

$$
\begin{aligned}
& \varepsilon_{g}^{A B}=\sum_{c}\left(R_{g}^{-1}\right)^{A C} \varepsilon_{c} R_{g}{ }^{C B} \\
& \left(\varepsilon_{g}^{-1}\right)^{A B}=\sum_{c}\left(R_{g}^{-1}\right)^{A C} \frac{1}{\varepsilon_{c}} R_{g}^{C B} \\
& G_{g h}^{A B}=\sum_{C . D}\left(R_{g}^{-1}\right)^{A C} \tilde{G}_{g h}^{C D} R_{h}^{D B}
\end{aligned}
$$

In reality we need not carry out these transformations, if we want to solve our eigenvalue problem. We can start directly with the equation (38) working from the beginning in the cubic system. (38) may be considered as a generalized eigenvalue problem of the form

$$
S C=w^{2} D \subseteq
$$


with

$$
\begin{aligned}
& S_{g h}^{A B}=G_{g h}^{A B}+\frac{1}{2} \delta_{g h} \varepsilon_{g}^{A B} \\
& D_{g h}^{A B}=\frac{1}{2} \delta_{g h}\left(\varepsilon_{g}^{-1}\right)^{A B}
\end{aligned}
$$

and

$$
\begin{aligned}
G_{g h}^{A B}= & \sum_{\underline{\mu}}^{1} e^{i \xi \underline{r}_{i i}^{0}}\left\langle A 0\left|W_{i j}\right| O B\right\rangle \\
\varepsilon_{g}^{A B}= & \bar{T}_{i}^{A B}-\delta^{A B} \bar{T}_{i} 00 \\
& +\sum_{j}^{\prime}\left[\left\langle A 0\left|W_{i j}\right| B O\right\rangle-\delta^{A B}\left\langle 00\left|W_{i j}\right| 00\right\rangle\right]
\end{aligned}
$$

For the explicite calculation we only need the equations (42) (46).

Since $D$ is not a unit matrix, the eigenvectors $\subseteq$ do not form an orthogonal system. Nevertheless the eigenvalues $\omega^{2}$ are real, if $D$ is positive definite. This is the case here, because the elgenvalues of $D$ are equal to $1 /\left(\begin{array}{ll}2 & \varepsilon_{A}\end{array}\right)$ with all $\varepsilon_{A}$ being positiv.

After these calculations which concern the ordered phase of $\mathrm{O}-\mathrm{H}_{2}$ and $p-D_{2}$, let us consider $p-H_{2}$ and $0-D_{2}$ for a moment.

These are special cases of the general problem (42): The interaction between the molecules is spherical symmetric and there are no orientational excitations. Thus the indices $A, B$ may be replaced by $K, L$. 
In the hypothetic fcc-phase of $p-H_{2}$ and $0-D_{2}$ the transformation (33) is not necessary, since the excitation energies $\varepsilon_{k}$ are all degenerate. (42) is no longer a generalized eigenvalue problem, because $D$ becomes proportional to the unit matrix. We get

$$
\begin{aligned}
& S_{g h}^{k L}=G_{g h}^{k L}+\frac{1}{2} \delta_{g h}^{k L} \cdot \varepsilon \\
& D_{g h}^{k L}=\frac{1}{2 \varepsilon} \delta_{g h}^{k L}
\end{aligned}
$$

The division into four sublattices is only formal, because there is no orientational order which distinguishes different sublattices from each other. Nevertheless we use four sublattices in order to compare with the ordered phases.

The real lattice of $p-\mid t_{2}$ and $0-\partial_{2}$ is $h c p$ and has two molecules per unit cell. We choose a coordinate system such that the z-axis is parallel to the c-axis of the hcp lattice. Then the two other axes lie in the hexagonal plane. As the z-axis is distinguished from the others, $\varepsilon_{z}$ will be different from $\varepsilon_{x}$ and $\varepsilon_{y}$. The latter must be equal, because the rank of the tensor $\varepsilon_{g}{ }^{k L}$ is two but the symetry of the z-axis is threefold. Thus $D$ is diagonal but not proportional to the unit matrix.

After the consideration of these special cases we go back to the general problem (42). We have to test whether the translational invariance of the lattice is actually fulfilled by (42). The limit of long wavelengths $(k \rightarrow 0)$ corresponds to a translation of the whole crystal, whereby the energy of the acoustical modes must go to zero.

For this case we shall see later that the translations and the librations are decoupled and therefore (42) becomes

$$
\sum_{L, L} S_{g h}^{k L}(k=0) C_{h}^{L}=0
$$


This must be fulfilled for any translation, hence

$$
S_{g h}^{k L}(\underline{k}=0)=0
$$

By (43) we obtain the following condition for the translational invariance (we shortly call it acoustical condition)

$$
\varepsilon_{g}^{k L}=-2 \sum_{j}^{\prime}\left\langle k 0\left|W_{i j}\right| 0 L\right\rangle
$$

On the other hand $\varepsilon_{g}^{k L}$ has been defined by $(46)$ :

$$
\varepsilon_{g}^{k L}=T_{i}^{k L}-\delta^{k L} T_{i}^{00}+\sum_{j}^{\prime}\left[\left\langle k 0\left|W_{i j}\right| L 0\right\rangle-\delta^{k L}\left\langle 00\left|W_{i j}\right| 00\right\rangle\right](52)
$$

The question is whether (51) and (52) will yield the same $\mathcal{E}$. Let us consider a normal crystal: Here the potential $W_{i j}$ behaves in such a way that the molecular field $V_{i}$ may be replaced by an harmonic oscillator potential. Then the single particle wavefunctions are Gaussians and the matrix elements in $(51,52)$ follow the relation (see chapter VII):

$$
\left\langle k_{0}\left|W_{i j}\right| 0 L\right\rangle=\delta^{k L}\left\langle 00\left|W_{i j}\right| 00\right\rangle-\left\langle k_{0}\left|W_{i j}\right| L_{0}\right\rangle
$$

Comparing (51) and (52) we obtain the condition

$$
\overline{1}_{i}^{k L}-\overline{1}_{i}^{00} \delta^{k L}=\sum_{j}^{1}\left[\left\langle k 0\left|W_{i j}\right| L_{0}\right\rangle-\delta^{k L}\left\langle 00\left|W_{i j}\right| 00\right\rangle\right]
$$


As a simplification we consider a spherically symetric molecular field $V_{i}$. The off-diagonal elements vanish and (54) simplifies to

$$
\begin{aligned}
& \bar{I}^{k k}-\overline{1}^{k 00}={\overrightarrow{L_{j}}}^{1}\left[\left\langle k 0\left|w_{i j}\right| k 0\right\rangle-\left\langle 00\left|w_{i j}\right| 00\right\rangle\right] \\
& T_{i}^{k k}-T_{i}^{00}=V_{i}^{k k}-V_{i}^{00}
\end{aligned}
$$

This is indeed fulfilled because of the wellknown fact that for an harmonic oscillator the kinetic and the potential energy are equal on the average.

We can conclude that the acoustical condition (51) is fulfilled if we use a harmonic model potential.

In this work we use a different method. We determine the ground state single particle wavefunction variationally by minimizing the Hartree ground state energy

$$
\begin{aligned}
E_{0} / N & =\overline{1}_{i}^{00}+\frac{1}{2} \vec{i}_{j}^{\prime}\left\langle 00\left|W_{i j}\right| 00\right\rangle \\
& =\overline{1}^{00}+\frac{1}{2} V_{i}^{00}
\end{aligned}
$$

As trial wave function we use a Gaussian:

$$
\varphi_{i}^{0}=\left(\frac{\gamma}{i 1}\right)^{3 / 4} e^{-\frac{1}{2} \gamma\left(\underline{R}_{i}-\underline{R}_{i}^{0}\right)}
$$

For the translational single particle excitations we then have to use 


$$
\begin{gathered}
-54- \\
\varphi_{i}^{x}=-\sqrt{2 \gamma}\left(X_{i}-X_{i}^{0}\right) \cdot \varphi_{i}^{0} \\
\varphi_{i}{ }^{0}, \varphi_{i}^{z} \quad \text { analogous }
\end{gathered}
$$

The molecular field $V_{i}$, which is obtained by the variation procedure, is not exactly an oscillator potential. Thus the functions $\varphi_{i}{ }^{\prime}, \varphi_{i}^{k}$ are not the exact eigenfunctions. (For example $V_{i}$ may be very similar to a parabola where $\varphi_{i}^{0}$ is large, but not where $\varphi_{i} k$ is large).

Therefore we cannot expect that (51) and (52) will give exactly the same $\mathcal{E}$. But for a normal crystal the difference will be very small.

For quantum crystals however the situation is more difficult. Nosanow has shown [29] that these crystals can formally be treated as normal crystals to a certain extent if the true interaction is replaced by an effective one $(I I, 16)$. For the case of helium Nosanow calculated numerically both $V_{i}$ and $\varphi_{i}^{\circ}$. At least near its minimum, $V_{i}$ looks very much like a parabola. Thus $\varphi_{i}{ }^{0}$ could be very well approximated by a Gaussian $(I I, 15)$. But it is not clear how much $V_{i}$ is parabolic, where $\varphi_{i}{ }^{k}$ is large. The comparison of (51) with (52) is a good test for this question.

Therefore we proceed in the following way: Since we know that the crystal is actually invariant against translations we use (51) Instead of (52) for the solution of the eigenvalue problem (42) in order to ensure the translational invariance. This means we calculate $\varepsilon_{g}^{k L}$ by the matrix elements $\left\langle k 0\left|W_{i j}\right| O L\right\rangle$ which are proportional to the coupling parameters. On the other hand we must also calculate $\varepsilon_{j}{ }^{k L}$ by $(52)$; as a sum of kinetic and potential energies. The comparison of the results of (51) and (52) will show, whether it is justified to treat not only the ground state but also the lowest excited states formally by the harmonic theory. 
We have discussed our method for quantum crystals in general so far. For the ordered phase of $0-H_{2}$ and $p-\partial_{2}$ there are additional diffculties.

For $P_{i}^{0}$ we use the isotropic Gaussan $(I I, 15),(58)$. The small anisotropic interaction, which is contained in $W_{i j}$, leads to a molecular field which is slightly anisotropic:

$$
V_{i}=\sum_{i}^{1} \int \omega_{i j} \varphi_{i}^{02} d v_{i}
$$

In a self consistent determination of $\varphi_{i}{ }^{0}$ the next step would be to solve the Hartree equation

$$
\left(T_{i}+V_{i}\right) \varphi_{i}^{0}=E_{i}^{0} \varphi_{i}^{0}
$$

This would lead to an anisotrnnic $\varphi_{i}{ }^{\circ}$, which would have to be inserted into $(60)$ again, and so on * .

We neglect this feedback of $V_{i}$ on $\varphi_{i}{ }^{0}$. This means that we calculate $\varphi_{i}^{0}$ in the zeroth order but all matrix elements in the first order with respect to the smallness of the anisotropic interaction compared with the total $W_{i j}$. Thus $\varphi_{i}{ }^{0}$ does not reflect the special point symmetry of the $\mathrm{Pa} 3$ space group, but the energies $\omega(k)$ of the collective excitations do.

* The self consistent determination of $\varphi_{i}^{0}$ by iteration of (60) with $(61)$ is identical with the variational determination of $\varphi_{i}^{0}$ by minimizing the Hartree ground state energy (57). As variational ansatz for an anisotropic $\varphi_{i}{ }^{0}$ we would have to use $(I I, 20)$. 
The lack of self consistency, which occurs by the use of the isotropic $\varphi_{i}{ }^{0}$, can be seen for example by considering (52). Here the kinetic part is diagonal because of $T_{i}^{k L} \sim \delta^{k L}$ but the rest, $V_{i}^{k L}-\delta^{k L} V_{i}{ }^{00} \quad$, is not. However this difficulty vanishes formally, since we have to use (51) instead of (52) anyhow, because of the condition for the translational invariance. 
VI Effective Hamlitonian for systems with short range correlations

In the last chapter we have diagonalized the Hamiltonian $H=T+V$ in the Random Phase Approximation. The description of the ground state and of the excited states has been based on the single particle functions $\psi_{i}{ }^{0}(I, 7)$ and $\psi_{i}{ }^{A}(V, 2,3)$.

For quantum crystals the short range correlations between the molecules become very important. Their influence can be taken into account by introducing Jastrow factors which are two particle functions $f_{i j}$ (II, 14, 21) into the wavefunctions. For the ground state we use

$$
\phi_{0}=\prod_{i}^{N} \varphi_{i}^{0} \prod_{i<i} f_{i j}
$$

$\begin{aligned} & \text { For the ground state energy } \\ & \text { pansion }(I I, 6) \text { has been made. }\end{aligned} \quad E_{0}=\frac{\left(\phi_{0}, H \phi_{0}\right)}{\left(\phi_{0}, \phi_{0}\right)}$ a cluster exConsequently for the consideration of the excitations in quantum crystals a cluster expansion of an effective Hamiltonian $\mathcal{X}=T+W$ has to be made. This has been carried out already by Biem [34] . We shortly cite the method and the results.

The total wavefunction of the excited quantum crystal is built up as a linear combination

$$
\phi=\sum_{\mu} c_{\mu} \phi_{\mu}
$$

of the functions

$$
\phi_{\mu}=\prod_{i}^{N} \varphi_{i}^{\mu_{i}} \bar{l}_{i<j} f_{i j}
$$

with the abbreviation

$$
\mu=\left\{\mu_{1}, \mu_{2}, \cdots \mu_{i} \cdots \mu_{N}\right\}
$$


$-58-$

where $\mu_{i}$ labels the ground state and the excited states of the molecule $i$. At the moment we consider only the translational excitations $\mu=x, y, z, \ldots \quad$. The generalization for the orientational excitations is straight forward, as we shall see later.

The eigenvalue problem

$$
H \phi=E \phi
$$

has to be solved. As the $\phi_{\text {fuN }}$ are not orthogonal, an overlap matrix

$$
\bar{F}_{\mu \underline{v}}=\left(\phi_{F}, \phi_{\underline{v}}\right)
$$

has to be introduced. The matrix elements of $H$ are

$$
H_{\mu \underline{\underline{v}}}=\left(\phi_{\underline{\mu},} H_{\underline{\underline{v}}}\right)
$$

Now the eigenvalue problem has the form

$$
\sum_{\underline{\nu}}\left(H_{\mu \underline{\underline{v}}}-\underline{E} \bar{F}_{\mu \underline{\underline{v}}}\right) C_{\underline{\underline{v}}}=0
$$

with the secular equation

$$
\operatorname{det}\left(H_{\mu \underline{v}}-E F_{\mu \underline{v}}\right)=0
$$


or

$$
\operatorname{det}\left(\mathcal{H}_{\mu \underline{v}}-E \delta_{\mu \underline{v}}\right)=0
$$

with

$$
X_{\mu \underline{\underline{v}}}=\sum_{\underline{\xi}}\left(F^{-1}\right)_{\mu \underline{\underline{I}}} H_{\underline{\underline{I}}}
$$

$X=F^{-1} \cdot H$ is a normal operator with real eigenvalues, because $F$ and $H$ are both hermitian and the overlap matrix can be assumed to be positive definite.

The matrices $H_{\mu \underline{\underline{v}}}, \mp_{\mu \underline{y}}$ and $X_{\mu \underline{\underline{y}}}$ represent $N$-particle operators. A cluster expansion is performed such that $\mathcal{H}$ is represented by a series of one, two, and more particle operators. The series is written down explicitely for all parts containing one and two particle operators. This means that only such matrix elements occur for which $\mu$ and $\underline{\nu}$ differ at most at two lattice sites $i$ and $j$.

Finally the effective Hamiltonian is given in the notation of second quantization:

$$
\begin{aligned}
& X=\sum_{\substack{\mu_{i}, v \\
j}}\left\langle\mu\left|P_{i}\right| \nu\right\rangle b_{i}^{\mu+} b_{i}^{\nu} \\
& +\frac{1}{2} \sum_{\substack{\mu, v_{1} \xi_{i} \\
i, j}}^{\prime}\left\langle\mu \xi\left|W_{i j}\right| v \eta\right\rangle b_{i}^{\mu+} b_{j}^{j+} b_{j}^{\eta} b_{i}^{\nu}+\ldots
\end{aligned}
$$

The operators $b_{i}^{\mu+}, b_{i}^{\mu}$ create resp. destruct particles in 
the states $\varphi_{i}^{\mu}$. $W_{i j}$ is an effective potential:

$$
\begin{aligned}
& {\left[W_{i j}\right]=\left[f_{i j}^{2}\right]^{-1} \cdot\left[f_{i j}^{2} \bar{V}_{i j}\right]} \\
& \quad+\left[f_{i j}^{2}\right]^{-1} \cdot\left[\left(P_{i}+P_{i}\right) f_{i j}^{2}\right]-\left[P_{i}+P_{j}\right]
\end{aligned}
$$

Here $[\cdots]$ denotes a matrix with the elements $\langle\mu \xi|\ldots| v \xi\rangle$.

In order to make the effective Hamiltonian practicable the matrix multiplications in (13) have to be understood as truncated multiplications in a subspace of low lying states. In our case it is consequent to use the space which is formed by the single particle ground state $\mu=0$ and the low lying excitations $\mu=x, y, z$ only.

$$
\bar{V}_{i j}=V_{i j}-\frac{\hbar^{2}}{2 M} \nabla_{r_{i j}}^{2} \ln f_{i j}
$$

has been used already in the cluster expansion for the ground state energy $(I I, 12)$.

$P$ is a differential operator

$$
P_{i} f_{i j}^{2}=-\frac{\hbar^{2}}{8 M}\left(2 \nabla_{i} f_{i j}^{2} \nabla_{i}+\Delta_{i} f_{i j}^{2}+f_{i j}^{2} \Delta_{i}\right)
$$

Here the sperators $\nabla_{i}$ and $\Delta_{i}$ act on all functions which are to the right of them. In the special case that $P_{i}$ acts on 1 instead on $f^{2}$ the kinetic energy operator is obtained:

$$
P_{i} \cdot 1=-\frac{\hbar^{2}}{2 M} \Delta_{i}=T_{i}
$$


Thus $P_{i}$ may be replaced by $T_{i}$ in the one particle part of the effective Hamiltonian (12). The same holds for the matrix $\left[P_{i}+P_{j}\right]$ in (13).

For the calculation of matrix elements a more suitable form of $P$ can be obtained by partial integration:

$$
P_{i} f_{i j}^{2}=-\frac{\hbar^{2}}{8 m}\left(\overleftarrow{\Delta}_{i} f_{i j}^{2}+f_{i j}^{2} \vec{\Delta}_{i}-2 \overleftarrow{\nabla}_{i} f_{i j}^{2} \vec{\nabla}_{i}\right)
$$

In this very symmetric form the operators act only into the direction of the arrows and do not affect $f^{2}$.

Let us see now how to use Biem's results for our problem.

In the last chapter we have considered systems without short range correlations. We have diagonalized an Hamiltonian of exactly the same type as (12) using the Random Phase Approximation or time dependent Hartree approximation. The only formal difference is that for systems with short range correlations $W_{i j}$ is not the true interaction but the complicated effective interaction given by (13). Thus we can use the results of the last chapter, if we replace everywhere the matrix elements of the potential $W_{i j}$ by the matrix elements (13).

The orientational excitations in the ordered state of $0-H_{2}$ and $p-\lambda_{2}$ can be included into our formalism by a straight forward generalization: We replace the single particle functions $\varphi_{i}{ }^{\circ}\left(\underline{R}_{i}\right)$ and $\varphi_{i}{ }^{k}\left(\underline{R}_{i}\right) \quad\left(K=x_{i} y_{1} z\right)$ by the functions $\psi_{i}{ }^{0}\left(\underline{R}_{i}, \Omega_{i}\right)$ and $\psi_{i} A\left(\underline{R}_{i}, R\right.$. which have been defined in $(I, 7)$ and $(V, 2.3)$. This is possible because the differential operators in $(12,13)$ act only on $R_{i}$ but not on $R_{i}$. As in the last chapter the index $\mu$ labels the single particle states:

$$
\mu=0_{i} A=0_{i} k_{i} \alpha=0_{i} x_{1} y_{1} z_{i}+1,-1
$$


For the generalized eigenvalue problem $(v, 42)$ we have to calculate the matrix elements $\left\langle\mu_{0}\left|W_{i j}\right| \gamma_{0}\right\rangle$ and $\left\langle\mu_{0}\left|W_{i j}\right| 0 \nu\right\rangle$ by (13). Since we use only $|00\rangle,|\mu \nu\rangle,|0 \mu\rangle$ as intermediate states in the matrix multiplications which occur in (13), we have to form the inverse matrix of $\bar{F}=\left[f_{i j}\right]$ within this restricted space.

Since $f_{i j}^{2}$ does not depend on orientations, the inversion must be performed actually only in the suspace of the translational states

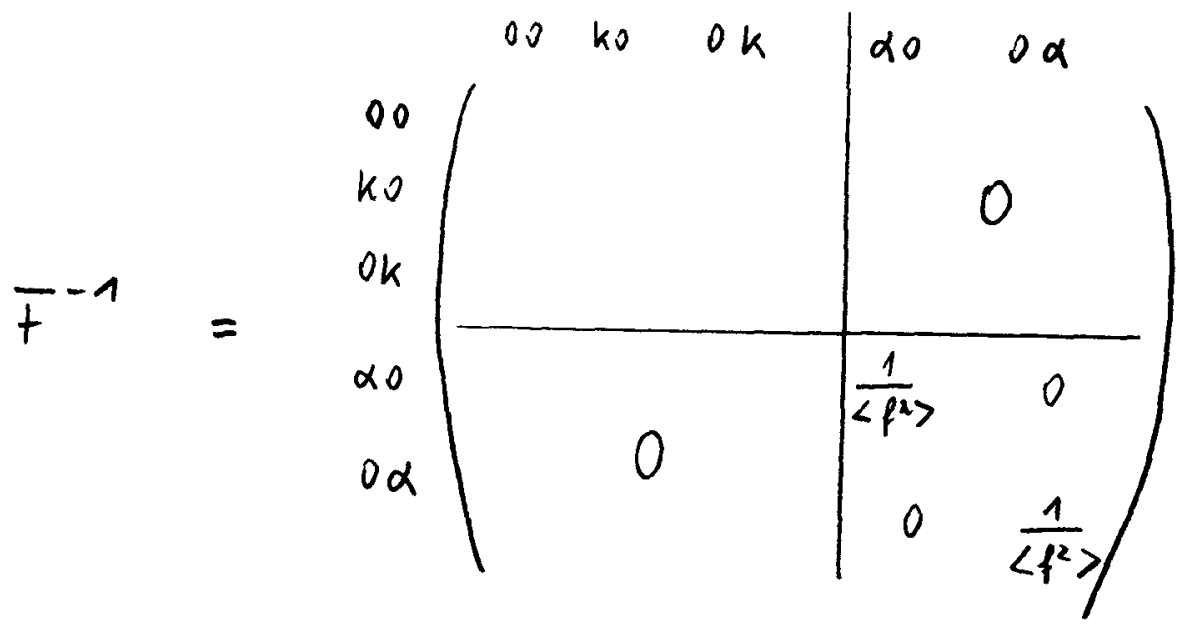

with $\left\langle f^{2}\right\rangle=\left\langle 00\left|f_{i j}^{2}\right| 00\right\rangle$

The same zeros as in (19) occur in the matrices $\left[\left(P_{i}+P_{j} \mid f_{i j}^{2}\right]\right.$ and $\left[P_{i}+P_{i}\right]$

$\left[W_{i j}\right]$ has been defined in (13) as a product of hermitian matrices. Thus $\left[W_{i j}\right]$ is generally not hermitian. Only in the subspace of the orientational states it is, because the matrix multiplications in (13) reduce here to a simple scalar multiplication, see (19).

In the subspace of the tranlational states the dexkviations from the symmetry numerically will turn out to be very small; they are of the 
same order as the numerical accuracy of the calculation of $\left[W_{i j}\right]$ and thus can be neglected.

The deviations are large however for elements like $\langle\alpha 0|W| O K\rangle$ which describe the coupling between librons and phonons. Compare 3.9 .

$$
\langle\alpha 0|W| 0 k\rangle=\left\langle\alpha_{0}\left|F^{-1}\right| \alpha_{0}\right\rangle \cdot\left\langle\alpha_{0}\left|V_{\text {eff }}\right| 0 k\right\rangle
$$

with

$$
\langle 0 k|W| \alpha 0\rangle=\sum_{L}\left\langle 0 k\left|F^{-1}\right| \begin{array}{l}
L_{0 L} 0 \\
00
\end{array}\right\rangle\left\langle\begin{array}{l}
L_{0} \\
0.0 \\
00
\end{array}\left|V_{\text {eff }}\right| \alpha 0\right\rangle
$$

Nevertheless the eigenvalues of the effective Hamiltonian (12) are real, as was mentioned already on page 59 .

We now specialize the tranlational single particle functions

$\varphi_{i}{ }^{0}, \varphi_{i} K$ in the same manner as we did in $(V, 58,59):$ For $\varphi_{i}^{0}$ we use a spherical Gaussian; and $\varphi_{i}{ }^{x}=-\sqrt{2 r}\left(X_{i}-X_{i}^{0}\right), \varphi_{i}^{0} ; \varphi_{i}, \varphi_{i} z$ analogous.

With these functions the second part of $W_{i j}$ in (13), the two body term of the kinetic energy, simplifies considerably:

$\left[P_{i}+P_{i}\right]$ is diagonal with the diagonal elements

$$
\begin{aligned}
& \left\langle 00\left|P_{i}+P_{j}\right| 00\right\rangle=\frac{\hbar^{2}}{2 M} 3 \gamma \\
& \left\langle k 0\left|P_{i}+P_{j}\right| k 0\right\rangle=\frac{\hbar^{2}}{2 M} 4 \gamma
\end{aligned}
$$

$\left[\left(p_{i}+p_{j}\right) f_{i j}^{2}\right]$ can be calculated by the symmetric form (17) of $P f^{2}$. Using the properties of the Gaussians it turns out after quite a lot of partial integrations that $\left[\left(p_{i}+p_{i} \mid f_{i j}^{2}\right]\right.$ can be expressed by the elements of $F$ : 
$\langle 00|\left(P_{i}+P_{j}\left|f_{i j}^{2}\right| 00\right\rangle=\frac{\hbar^{2}}{2 m} 3 \gamma\left\langle 00\left|f_{i j}^{2}\right| 00\right\rangle$

$\langle k 0|\left(P_{i}+P_{i}\left|f^{2}\right| L_{0}\right\rangle=\frac{\hbar^{2}}{2 M} \gamma\left[3\left\langle k 0\left|f^{2}\right| L O\right\rangle+\delta_{k L}\left\langle 00 \mid f^{2} 100\right\rangle\right]$

$$
\begin{aligned}
& \langle k 0|\left(P_{i}+p_{i}\left|f^{2}\right| O L\right\rangle=\frac{\hbar^{2}}{2 M} 3 \gamma\left\langle k 0\left|f^{2}\right| 0 L\right\rangle \\
& \langle k 0|\left(P_{i}+p_{i}\left|f^{2}\right| 00\right\rangle=\frac{\hbar^{2}}{2 M} 3 \gamma\left\langle k 0\left|f^{2}\right| 00\right\rangle
\end{aligned}
$$

Therefore the calculation of $\left[W_{i j}\right]$ has been reduced to the calculation of $F=\left[f_{i j}^{2}\right]$ and $\left[\gamma_{e f}\right]=\left[f_{i j}^{2} \bar{V}_{i j}\right]$, which will be performed explicitely in the next chapter.

Before we do this let us treat the ground state by the effective Hamiltonian (12). The ground state energy is obtained by

$$
\begin{aligned}
& E_{0}=\left\langle\pi_{i} \psi_{i}^{0}|H| \pi_{i} \psi_{i}^{0}\right\rangle \\
& =\sum_{i}\left\langle 0\left|T_{i}\right| 0\right\rangle+\frac{1}{2} \sum_{i, j}^{\prime}\left\langle 00\left|W_{i j}\right| 00\right\rangle \\
& =\sum_{i}^{L}\left\langle 0\left|T_{i}\right| 0\right\rangle+\frac{1}{2} \sum_{i_{i j}}^{\sum_{i j}^{\prime}}\left\langle 00\left|\left[f_{i j}^{2}\right]^{-1} \cdot\left[f_{i j}^{2}-\bar{V}_{i j}\right]\right| 00\right\rangle
\end{aligned}
$$

for the two body term of the kinetic energy vanishes.

(24) is identical with Nosanow's cluster expansion of $E_{0}$ up to the two body terms (II, 6 - 10), if only $|00\rangle$ is used as intermediate state for the matrix multiplication. But since we use $|00\rangle,\left|\mu_{0}\right\rangle,|0 \mu\rangle$ for the calculation of $\left\langle\mu 0\left|W_{i j}\right| \gamma_{0}\right\rangle$ and $\left\langle\mu 0\left|W_{i j}\right| 0 \nu\right\rangle$, we have to do the same for $\left\langle 00\left|W_{i j}\right| 00\right\rangle$. Otherwise we would calculate the excitation energies $\varepsilon_{k}=E^{k}-E^{0}$ as a difference of two quantities obtained by different approximations. 
Consequently for the ground state variation we have to take the approximation (22) instead of Nosanow's $E_{0}$. But it turns out by the numerical calculation that the off-diagonal elements $\left\langle 00\left|F^{-1}\right| 0 \mathrm{k}\right\rangle$ which cause the differences between $(22)$ and Nosanow's $E_{0}$, are rather small $(0.048-28$ of the diagonal elements for the different cases we consider). Therefore it is a good approximation to take the parameters which have been obtained by the variation of Nosanow's $E_{0}$ for the calculation of the excitations. This is a considerable simplification.

\section{Calculation of matrix elements}

In order to obtain the matrix elements $\left\langle A O\left|W_{i j}\right| B 0\right\rangle$ and $\left\langle A O\left|W_{i j}\right| O B\right\rangle$ we have to calculate the elements $\langle A 0|\cdots| B 0\rangle,\langle A 01 \cdots| 0$ B $\rangle$, $\langle A O|\cdots| 00\rangle$ of the matrices $\left[f^{2}\right]$ and $\left[V_{e n}\right]$. Analogously to the elements $\langle 001 \cdots 100\rangle$, which have been calculated in chapter III, we have to separate the translational integrations from the orientational ones. Therefore we start directly with the equations (III, 12 - 15), where $V_{\text {ef }}$ has been transformed to the fixed frame with the axis $\pm_{i j}^{0}$.

$$
\begin{aligned}
V_{\text {eff }}= & f^{2}\left(r_{i j}\right) \cdot \overline{V^{0}}\left(r_{i j}\right)+f^{2}\left(v_{i j}\right) V^{1}\left(r_{i j}\right) \cdot U\left(\Omega_{i} \underline{r}_{0}, \Omega_{i} \underline{v}_{0}, R_{i j}\right) \\
& +f^{2}\left(r_{i j}\right) \Gamma\left(r_{i j}\right) \cdot Q\left(\Omega_{i} \underline{r}_{i}, R_{j} \underline{r}_{0}, \Omega_{i j}\right)
\end{aligned}
$$


The matrix $\left[f^{2}\right]$ will be treated in the following as a special case of $\left[f^{2} \bar{V}^{0}\right]$ setting $\bar{V}_{0} \equiv 1$.

a) For the case $A=\alpha, B=\beta ; \alpha, \beta= \pm 1$ the separation can be performed in exactly the same manner as in chapter III. We obtain

$$
\begin{aligned}
& \left\langle\alpha_{0}\left|f^{2} \overline{v^{0}}\right| \beta_{0}\right\rangle=\delta_{\alpha \beta} \cdot\left\langle 00 \mid f^{2} \overline{v^{0}} 100\right\rangle \\
& \left\langle\alpha_{0}\left|f^{2} \overline{v^{0}}\right| \theta_{\beta}\right\rangle=\left\langle\alpha_{0}\left|f^{2} \overline{v_{0}}\right| 00\right\rangle=0
\end{aligned}
$$

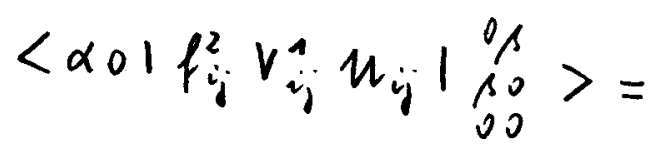

$$
\begin{aligned}
& \left\langle\left.\alpha_{0}\left|u_{i j}{ }^{r_{0}}\right|\right|_{s_{0}} ^{0}\right\rangle=\left\langle 00\left|f_{i j}^{2} V_{i j}^{1} \tilde{P}_{2}\left(g_{i j}\right)\right| 00\right\rangle \\
& \left\langle\alpha_{0}\left|f_{i j}^{2} \Gamma_{i j} Q_{i j}\right| \underset{\substack{\Delta_{j} \\
j o}}{\rho_{0}}\right\rangle= \\
& \left\langle\alpha 0\left|Q_{i j} v_{0}\right| \gamma_{0 j}\right\rangle \cdot\left\langle 001 f_{i j} \Gamma_{i j} \tilde{P}_{u}\left(Q_{i j}\right) \mid 00\right\rangle
\end{aligned}
$$

The elements $\left\langle 00\left|p^{2} \bar{\gamma}^{2}\right| 00\right\rangle,\left\langle 00 \mid f^{2} V^{1} \hat{p}_{2} 100\right\rangle,\left\langle 00 \mid f^{2} \Gamma \hat{p}_{4} 100\right\rangle$ depend only on the shell number $S$ and have been calculated in

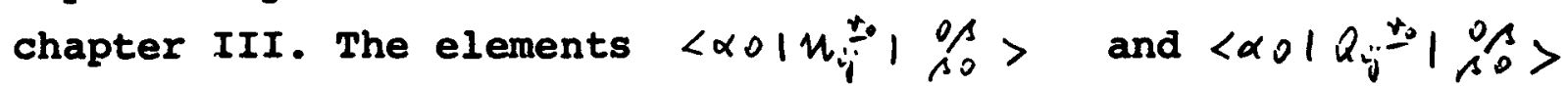
occurred already in the rigid lattice libron theory [18, 19] and were calculated there for nearest neighbours. $\left\langle\alpha 0\left|u_{i j}\right| 0 /\right\rangle$ vanishes because $u_{i j}$ is a sum of two parts which depend only on $i$ resp. $j$. Furthermore the sum of the elements $\left\langle\alpha_{0}\left|u_{i j}{ }_{0}\right| \beta_{0}\right\rangle$ vanishes for each shell of neighbours (compare with (III, 36)).

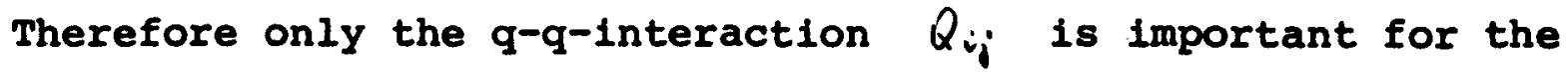
librons. By (4) we see that the results of the rigid lattice libron theory can be generalized to include the zero point motion of the molecules, when the rigid lattic q-q-coupling constant $\Gamma_{0}$ is replaced by the effective coupling constant $\left\langle 00\left|f^{2} r \tilde{P}_{4}\right| 00\right\rangle /\left\langle 00\left|f^{2}\right| 00\right\rangle$. 
The elements $\left\langle\alpha 0\left|Q_{i j} \underline{r}_{0}\right| 00\right\rangle$ and $\left\langle\alpha 0\left|u_{i j} \underline{v}_{0}\right| 00\right\rangle$ will be discussed later.

For the matrix elements with $A=K, B=L ; K, L=x, y, z$ and the ones with mixed librational and tranlational indices (e.g. $A=K$, $B=\alpha$ ) the separation does not lead to a simple product of matrix elements as in $(2-4)$ but to a sum of such products.

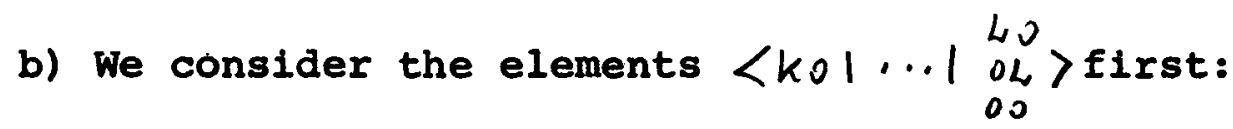

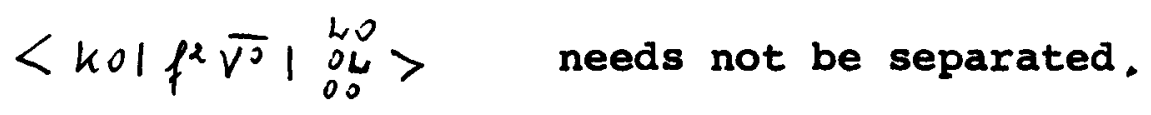

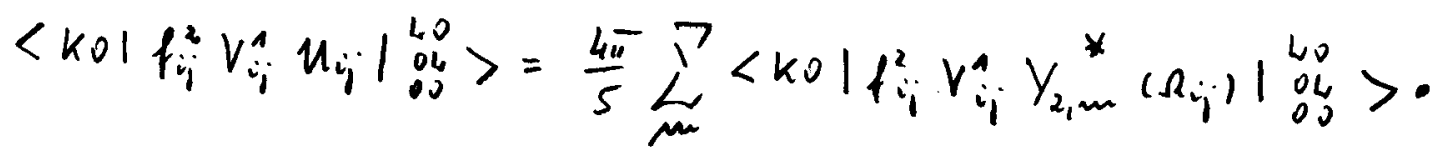

$$
\begin{aligned}
& \text { - }<001 Y_{2, m}\left(\Omega_{i} r_{0}\right)+Y_{2, m}\left(\Omega_{j} \underline{r}_{0}\right)|00\rangle
\end{aligned}
$$

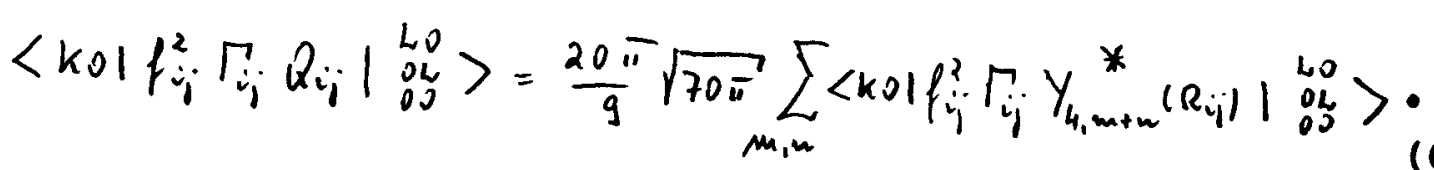

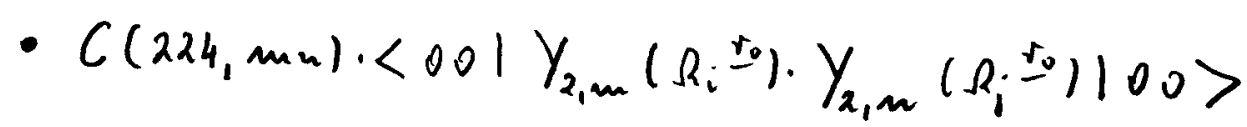

The orientational matrix elements can be calculated analytically using (XI, 5):

$$
\begin{aligned}
& \left\langle k o\left|f_{i j}^{2} v_{i j}^{1} U_{i j}\right|_{00}^{L O}{ }_{00}\right\rangle
\end{aligned}
$$

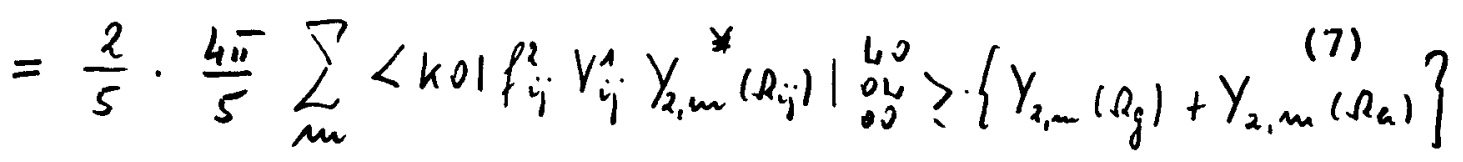




$$
\begin{aligned}
& \left\langle k 0\left|f^{2} \Gamma Q\right| \log _{00}^{L 0}\right\rangle \\
& =\frac{4}{25} \frac{20 \pi}{9} \sqrt{70 \pi} \sum_{m, m}\left\langle\left. k 01 f^{2} \Gamma Y_{4, m+m}^{*}\right|_{0 L} ^{L}{ }_{0 L}^{0}\right\rangle C(224, m n) Y_{2, m}\left(R_{g}\right) Y_{2, \omega}\left(\Omega_{n}\right)
\end{aligned}
$$

with

$$
\Omega_{g}=\Omega_{\underline{d}_{g}}^{\underline{r}_{0}} ; \quad \Omega_{h}=\Omega_{\underline{d}_{h}}^{\underline{r_{0}}}
$$

c) We still have to perform the separation for the elements $\left\langle k 01 \ldots \mid \begin{array}{c}\alpha 0 \\ 0 \alpha\end{array}\right\rangle$ :

$\left\langle k_{0}\left|f^{2} v^{\wedge} u\right|_{0 \alpha}^{\alpha 0}\right\rangle=\frac{4 \pi}{5} \sum_{m}^{-}\left\langle k 0\left|f^{2} V^{\wedge} y_{2, m}^{*}\right| 00\right\rangle$.

$$
.\left\langle 001 Y_{2, m}\left(R_{i}^{v_{\nu}}\right)+Y_{2, m}\left(R_{i}^{V_{0}}\right) \mid \begin{array}{c}
\alpha_{0} \\
0 \alpha
\end{array}\right\rangle
$$

$$
\begin{gathered}
\left\langle k_{0}\left|f^{2} \Gamma Q\right|_{0 \alpha}^{\alpha_{0}}\right\rangle=\frac{20 \pi}{g} \sqrt{70 \pi} \sum_{m, \omega}\left\langle k 0\left|f^{2} \Gamma Y_{4, m+w}^{*}\right| 00\right\rangle . \\
\cdot C(224, m n)\left\langle 001 Y_{2, m}\left(R_{i}^{r_{0}}\right) \cdot Y_{2, n}\left(R_{i} t_{0}\right) \mid \begin{array}{c}
\alpha_{0 \alpha} \\
0 \alpha
\end{array}\right\rangle
\end{gathered}
$$

Here the orientational matrix elements cannot be represented simply by spherical harmonics as in (5) and (6). We have to use the formula (XI, 3) 


$$
\begin{aligned}
& \left\langle m_{i}\left|Y_{2, \mu}\left(\Omega_{i} \underline{r}_{0}\right)\right| m_{i}^{\prime}\right\rangle= \\
& \frac{-1}{\sqrt{2 \pi}} C\left(121_{i} m_{i}^{\prime}, m_{i}-m_{i}^{\prime}\right) \cdot \partial_{m_{i}-m_{i}^{\prime}, \mu}^{2}\left(\alpha, s_{1}, \gamma\right)
\end{aligned}
$$

The functions $\left|m_{i}\right\rangle=Y_{1, m_{i}}\left(\Omega_{i} \underline{d}_{g}\right)$ refer to the $\underline{d}_{g}$ body diagonal, whereas $V_{2, \mu}\left(a_{i} v_{0}\right)$ refers to the intermolecular axis $\Psi_{i j}^{\circ}$. The Euler angles $\alpha_{1}, \gamma_{1} \gamma$ describe the rotation of the coordinate system with $\underline{d}_{g}$ as z-axis to the system with $t_{0}$ as z-axis.

For (9) and (10) special cases of (11) have been used; e.g. $m_{i}=0_{i} m_{i}^{\prime}=\alpha$. We do not write down the results explicitely.

Moreover with the aid of (11) the elements

$$
\begin{aligned}
& \left\langle\alpha_{0}\left|Q_{i j} \underline{v}_{0}\right| \text { sos }_{0 / \beta}\right\rangle= \\
& \frac{10}{3} \pi \sqrt{70} \sum_{\mu} C\left(22 L_{i} \mu_{1}-\mu\right)\left\langle\alpha_{0}\left|Y_{2, \mu}\left(\Omega_{i}{ }^{r_{0}}\right) Y_{\alpha_{1}-\mu}\left(R_{j}{ }^{\nu}\right)\right|_{0 \beta}^{\beta_{0}}{ }_{0 \beta}^{(12)}\right.
\end{aligned}
$$

can be obtained in a more general way than in the libron theory $[18,19]$. The calulation of $\left\langle\alpha 0\left|Q_{i j} t_{0}\right| 0,0\right\rangle$ and $\left\langle\alpha 0\left|u_{i j} v_{0}\right| 00\right\rangle$, which had been postponed in a), can also be performed by (11).

Now we have separated the tranlational integrations from the orientational ones for all matrix elements we are interested in. Furthermore we have performed the orientational integrations. We still have to calculate the translational matrix elements. These are of the type $\left\langle k o\left|g\left(t, \Omega_{i j}\right)\right| \substack{0.0 \\ j 0}_{j}\right\rangle$ with $g\left(\gamma_{,} \Omega_{i j}\right)$ representing one of the integrands $f^{2}, f^{2} v^{0}, f^{2} V^{*} y_{2, m}^{*}, f^{2} \Gamma y_{4, m+\cdots}^{*}$ where $h_{i j}=\Omega_{ \pm}^{r_{0}}$ is the position of \pm with respect to $t_{2}$. Analogously to (III, 20) we introduce center of mass and relative coordinates and obtain: 


$$
\left.\left\langle k_{0}|g| \begin{array}{l}
L 0 \\
0 L
\end{array}\right\rangle=\frac{1}{2} \delta_{k L}\langle 00|g| 00\rangle \pm\right]_{k L}
$$

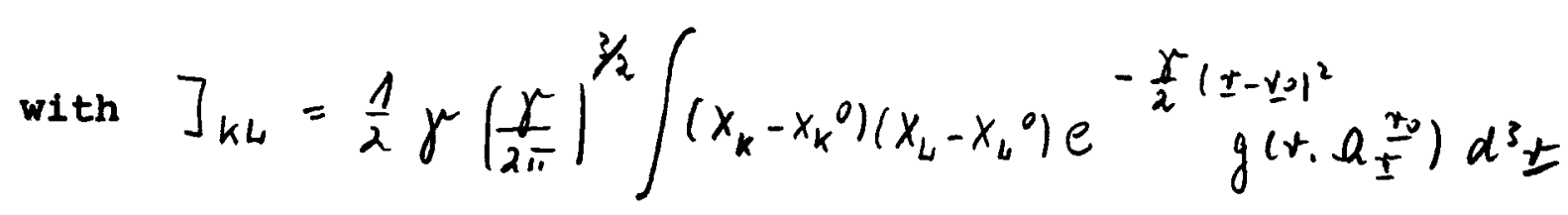

and

$$
\langle k 0 \lg \mid 00\rangle=]_{k}
$$

$$
=\sqrt{\frac{\gamma}{2}}\left(\frac{\gamma}{2 \pi}\right)^{3 / 2} \int\left(x_{k}-x_{k}^{0}\right) e^{-\frac{\theta}{2}\left( \pm-r_{0}\right)^{2}} g\left(r_{1} \Omega_{ \pm}^{\tau_{0}}\right) d^{3} \underline{ }
$$

with $I=\left\{x_{k}\right\}$

In (14) and (15) I refers to the crystal fixed coordinate system, in which we measure the wave vector $\underline{k}$. ( See $(v, 45))$. In order to be able to perform the integrations $(14,15)$ we must rotate the crystal fixed system so that to becomes the new z-axis:

$$
\underline{r}=M \underline{I} \text { with } \underline{r}_{0}=M \cdot\left(\begin{array}{l}
0 \\
0 \\
1
\end{array}\right)
$$

The rotation matrix $M$ can be found e.g. in [38].

By (16) the integrals J are represented as a sum of integrals ' '.

$$
\begin{aligned}
& \left.J_{k L}=\sum_{k, e} M_{k k} M_{L e}\right]_{k e}^{\prime} \\
& \left.J_{k}=\sum_{k} M_{k k}\right]_{k}^{\prime}
\end{aligned}
$$


]$_{k e}^{\prime}$ and $J_{k}^{\prime}$ are formally identical with (14) and (15), if $\underline{\Psi}$ is replaced by $\pm^{\prime}$. But the important difference is that in $J^{\prime} \pm_{0}^{\prime}$ is the $z^{\prime}$-axis; thus $\Omega_{I^{\prime}}^{r^{\prime}}=\left(\vartheta^{\prime}, \varphi^{\prime}\right)$ represents the polar angles of -'.

Moreover we notice that $I_{K L}$ and $I_{k}$ depend on $\underline{r}_{0}= \pm_{i j}{ }^{\circ}$, but $I_{k e}$ ' and $J_{k}^{\prime}$ depend only on $r_{0}^{\prime}=r_{0}$. Thus $I_{k c}^{\prime}$ and $I_{k}^{\prime}$ must be calculated only once for each shell of neighbours. $I_{k 4}$ and $J_{k}$ are obtained for each special nelghbour $j$ of this shell by the transformations (17).

As to the explicite calculation of $J_{k e}$ ' and $J_{k}$ we can introduce spherical coordinates $\tau^{\prime}, \vartheta^{\prime}, \varphi^{\prime}$. The angular integrations can be carried out analytically and only the radial integrations must be performed by numerical computation.

But in reality this procedure turns out to be not as practicable as in the ground state calculations. The angular integrations lead to very complicated expressions, which moreover are different for the different functions $g\left(r^{\prime}\right)$.

Therefore we use a more advantageous procedure. $I_{k e} e^{\prime}$ and $I_{k}{ }^{\prime}$ are computed in the form $(14,15)$ by 3 -dimensional numerical integrations * . Since for each sehell the integration region is the same for all integrands, we can compute $I_{k e}{ }^{\prime}$ and $J_{k}$ simultaneously with respect to the different functions $g$ and with respect to the indices $k$ and $l$. Because of $g=f^{2}, f^{2} v^{\overline{0}}, f^{2} v^{1} y_{2, m}$, $f^{2} \Gamma Y_{4, m+n}^{*}$ there are 16 different functions $g$. Thus in principle alltogether 16-12=192 integrals $J^{\prime}$ can be calculated simultaneously. This number is reduced to 66 , if we use the symmetries $J_{k e^{\prime}}=J_{e k}^{\prime}$ and $Y_{e_{1}{ }^{*}}^{*}=(-1) Y_{e_{1}-m}$ and the fact that the inte-
grals $]_{k e^{\prime}}$ with $g=f^{2} \Gamma Y_{4, m+n}^{*} \quad$ vanish for $|m+m|>2$ and that

We use the simplest method: The integration region is divided into a large number (e.g. 20000 ) of equal cubes; the integral is approximated by the sum of the function values at the middle points of the cubes multiplied by the volume of a cube. 
the integrals $J_{k}^{\prime}$ vanish for $|m|>1$ and $|m+n|>1$. The last statement can be proven by introducing spherical coordinates and by performing the $\varphi^{\prime}$-integration. - The symmetries and the vanishing integrals are used as tests of the numerical integration.

Now let us summarize what must be made in order to get the matrix elements $\langle A O|W| B O\rangle$ and $\langle A O|W| O B\rangle$, which occur in the eigenvalue problem $(V, 42)$ :

The rigid lattice data are calculated only once and in advance: For each neighbour $j \equiv(m, h)$ of a molecule $i \equiv(m, g)$ we need three sets of Euler angles which connect the $\underline{r}_{i j}{ }^{0}$-coordinate system 1) with the $\underline{d}_{g}$-system 2) with the $\underline{d}_{h}$-system, and 3) with the crystal fixed system. The orientational matrix elements are calculated as functions of the Euler angles 1) and 2).

The translational matrix elements are different for the different 1sotopes, potential parameters and SRC-functions. which we consider. For each shell of neighbours the integrals J $\mathrm{ke}^{\prime}$ and $\mathrm{J}_{\mathrm{k}}^{\prime}$ are computed simultaneously. The translational matrix elements are obtained then for each special neighbour of this shell by the transformation (17) using the Euler angles 3).

Then the elements of $\left[V_{y H}\right],\left[f^{2}\right]$, and $\left[\left(P_{i}+P_{j} \mid f^{2}\right]\right.$ are calculated by $(2)-(6),(9),(10),(V I, 21)$ and the inverse of $\left[f^{2}\right]$ is formed. [ $\left[W_{i j}\right]$ is obtained by the matrix multiplications in (VI, 13).

In order to get stable results for the excitation energies, [W $\left.W_{j j}\right]$ must be calculated at least for the first three shells of the fcc lattice. In this work the first five * shells have been taken into account. From the third shell $\left[f^{2}\right]$ can be replaced by the unit matrix.

respectively six shell of the hop lattice. 
The method which has been described till now is not the only one to calculate $\left[W_{i j}\right]$. There is another one, which is not suitable for the explicite calculation but which is very usefull for general considerations, for example concerning the symmetries of $\left[W_{i j}\right]$.

In this method we work from the beginning in the crystal fixed coordinate system, which we denote by the index $G$. We can take over all formulas of the first method, except those of part a), when we replace the index $I_{i j}^{\circ}$ by $C^{\prime}$ everywhere. In part a) we cannot use the simple formulas $(3,4)$, but we have to proceed in the same manner as in part b) and $c$ ). For each part we give the results of the separation for one typical matrix element:

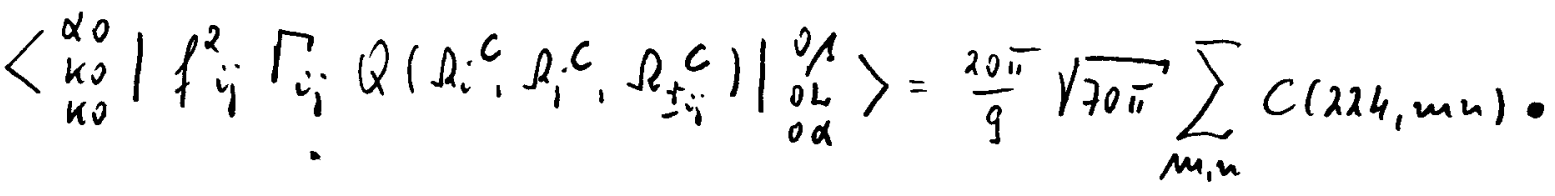

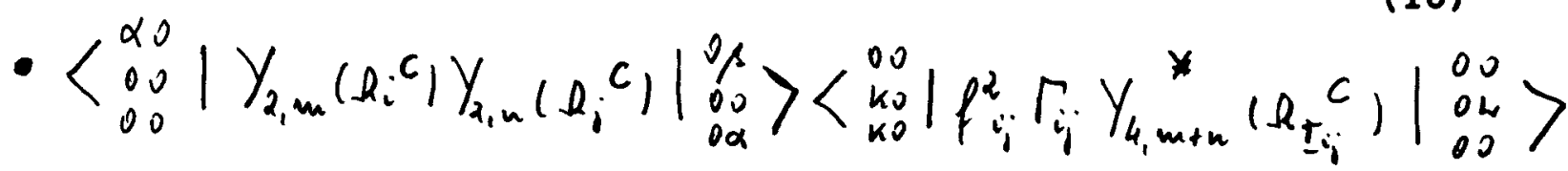

Again we use the formulas $(x I, 3,5)$ and we see that the orientational matrix elements are expressed by functions of the Euler angles, which connect the $\underline{d}_{g}$ - and $\underline{d}_{h}$ - system with the $G$-system. Thus these elements do not depend on $i$ and $j$, as in the first method, but only on $g$ and $h$.

The translational elements are again given by (13) - (15), where $\ell_{ \pm}^{t 0}$ must be replaced by $\Omega_{ \pm}^{C}$.

We want to consider the behaviour of the elements (18) under the symetry operations of the fcc lattice.

First we consider an inversion $\underline{r}_{i j}^{0} \rightarrow-\underline{r}_{i j}^{0}$. The orienta- 
tional elements in (18) do not change, because the sublattice indices $g$ and $h$ are not changed by the inversion. The tranlational elements $\left\langle\begin{array}{ll}k_{0} \\ 0\end{array}\left|f^{2} \Gamma Y_{4, m+n}^{*}\right|_{00}^{0 L}>\right.$ change nelther, but the elements $\langle k 0|\cdots| 00\rangle \quad$ change their sign, as can be seen looking at (13) - (15). These considerations can be generalized straightforwardly for all matrix elements of $\left[\mathrm{V}_{\mathrm{en}}\right]$ and $\left[\mathrm{f}^{2}\right]$, which have not been written down. We conclude that the elements $\langle\alpha 01 \ldots|$ so 0

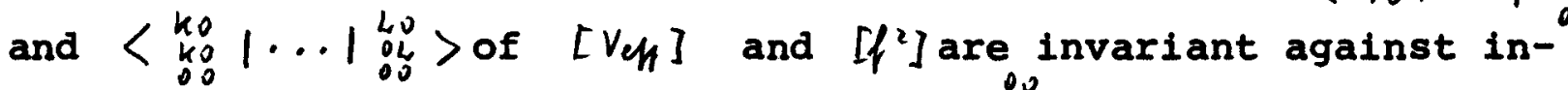
version, whereas the elements $\left\langle k 01 \ldots \mid \begin{array}{l}\alpha_{0}^{0} \\ 0 \alpha\end{array}\right\rangle$ change their sign. The same properties hold for $\left[W_{i j}\right]$ as can be seen by the definition (VI, 13).

Similarly other operations can be used which leave $g$ and $h$ unchanged. For cubic symmetry e.g. It can be shown that the sum $\sum_{\mu}^{\prime}\left\langle k o\left|W_{i j}\right| 0 L\right\rangle$ with $i$ belonging to sublattice 1 and $j$ be $\frac{m}{1}$ onging to sublattice 2 vanishes for $k=x, L=y$ and for $k=x, L=z$ but not for $k=Y, L=Z$. This relation and analogous ones will be used in the next chapter.

The sublattices have been labelled in $(I, 10)$ 
$-75-$

VIII Solution of the secular equation For $k=0$

The case $k=0$ will be discussed in detail on two reasons: It is of high symmetry, so the eigenvalue problem can be solved anallytidally. Furthermore the results can be compared with the existing optical measurements.

We want to solve the generalized eigenvalue problem $(v, 42)$ for the ordered state of $\mathrm{O}-\mathrm{H}_{2}$ and $p-D_{2}$.

$$
\begin{gathered}
S \subseteq=w^{2} D \subseteq \\
\text { with } S_{g h}^{A B}=\sum_{\underline{m}}^{1} e^{i \underline{k}_{-i}^{0}}\left\langle A 0\left|W_{i j}\right| 0 B\right\rangle+\frac{1}{2} \delta_{g h} \varepsilon_{g}^{A B} \\
D_{g h}^{A B}=\frac{1}{2} \delta_{g h}\left(\varepsilon_{g}^{-1}\right)^{A B} \\
i \equiv(m, g) ; j=(\underline{\mu}, h) \\
\varepsilon_{g}^{A B} \text { is given by }(v, 33-39): \\
\varepsilon_{g}^{k \alpha}=\varepsilon_{g}^{\alpha k}=0 \\
\varepsilon_{g}^{\alpha \beta}=\varepsilon_{\alpha} \cdot \delta^{\alpha \beta}
\end{gathered}
$$

with $\varepsilon_{\alpha}=\varepsilon_{\bar{\alpha}}(\bar{x}, 27,28)$ 
Because of (4.5) $D$ is diagonal in the subspace of the orientational excitations:

$$
D_{g h}^{\alpha \beta}=\frac{1}{\lambda \varepsilon_{\alpha}} \delta_{g h}^{\alpha / \beta}
$$

and

$$
D_{g h}^{k \alpha}=D_{g^{k}}^{\alpha k}=0
$$

For $\varepsilon_{g}^{k L}$ we have to use $(V, 51)$, as has been discussed in chapter $v$ :

$$
\varepsilon_{g}^{K L}=-2 \sum_{j}^{\prime}\left\langle k 0\left|W_{i j}\right| 0 L\right\rangle
$$

The symmetries of $\varepsilon_{g}^{K L}$ can be seen by the transformation $(v, 39)$ :

$$
\varepsilon_{g}^{k L}=\sum_{M}\left(R_{g}^{-1}\right)^{k M} \varepsilon_{M} R_{g}^{M L}
$$
$R_{g}$ rotates the coordinate system with the body diagonal $\underline{d}$ as
z-axis into the crystal fixed system $C$ :

$$
R_{g} \underline{d}_{g}=\left(\begin{array}{l}
0 \\
0 \\
1
\end{array}\right)
$$

Because the body diagonals are axes of threefold symmetry, the translational single particle excitation energies $\varepsilon_{x}$ and $\varepsilon_{y}$ are equal.

The $\underline{d}_{g}$ have been defined in $(I, 11)$. By $(9,10)$ we obtain e.g. for $g=1$ : 
$-77-$

$$
\varepsilon_{1}=\left(\begin{array}{ccc}
\varepsilon & e & e \\
e & \varepsilon & e \\
e & e & \varepsilon
\end{array}\right)
$$

with $\varepsilon=\frac{2}{3} \varepsilon_{x}+\frac{1}{3} \varepsilon_{z ;} \quad e=\frac{1}{3}\left(\varepsilon_{z}-\varepsilon_{x}\right)$

The other $\varepsilon_{g}$ are obtained by a simple transformation from one sublattice to another one:

$$
\varepsilon_{h}=R_{(g \rightarrow h)}^{-1} \varepsilon_{g} R(g \rightarrow h)
$$

with

$$
R(g \rightarrow h) \underline{d}_{g}=\underline{d}_{h}
$$

For example we get

$$
\varepsilon_{2}=\left(\begin{array}{ccc}
\varepsilon & -e & -e \\
-e & \varepsilon & e \\
-e & e & \varepsilon
\end{array}\right)
$$

The inverses of the $\varepsilon_{g}$ are used to construct the matrix

$$
D_{g h}^{k L}=\frac{1}{2} \delta_{g h}\left(\varepsilon_{g}^{-1}\right)^{k L}
$$


e.g.

$$
\frac{1}{2} \varepsilon_{1}^{-1}=\left(\begin{array}{lll}
\delta & d & d \\
d & \delta & d \\
d & d & \delta
\end{array}\right)
$$

with $\delta=\frac{1}{2} \frac{\varepsilon+e}{\varepsilon(\varepsilon+e)-2 e^{2}} ; d=-\frac{1}{2} \frac{e}{\varepsilon(\varepsilon+e)-2 e^{2}}$

The other $\varepsilon_{g}^{-1}$ are obtained similar to $(12,13)$.

After these general considerations we consider the special case

$\underline{k}=0$. In the last chapter it has been shown that $\left\langle\alpha_{0}\left|W_{i j}\right| O \beta\right\rangle$ and $\left\langle k_{0}\left|W_{i j}\right| O L\right\rangle$ are invariant against an inversion $\underline{r}_{i j}^{0} \rightarrow-\underline{r}_{i j}^{0}$, whereas the elements $\left\langle k 0\left|w_{i j}\right| 0 \alpha\right\rangle$ and $\left\langle\alpha \circ\left|W_{i j}\right| O k\right\rangle$ change their sign. Thus we have

$$
\sum_{\underline{\mu}}^{\prime}\left\langle k 0\left|W_{i j}\right| 0 \alpha\right\rangle=0
$$

and because of $(2,4)$

$$
S_{g_{h}}^{k \alpha}(\underline{k}=0)=S_{g h}^{\alpha k}(\underline{k}=0)=0
$$

Since these elements vanish, which couple the orlentational excitations with the translational ones, $S$ decays for $\underline{h}=0$ into an $8 \times 8$ submatrix $S \frac{\alpha}{g} h$ and a $12 \times 12$ submatrix $S_{g h}^{k}$. (The argument $\underline{k}=0$ is suppressed here and in the following). 
$S_{g / \alpha}^{\alpha}$ has been diagonalized already in the libron theories $[18,19]$ and needs not be discussed here.

For the discussion of $S_{g h}^{k l}$ we introduce the abbreviation

$$
\sum_{\underline{M}}^{\prime}\left\langle k 0\left|W_{i j}\right| O L\right\rangle=C_{g L}^{k L}
$$

Because of $(2,8)$ we can write

$$
S_{g h}^{k L}=C_{g h}^{k L}-\delta g h \sum_{h^{\prime}} C_{g h^{\prime}}^{k L}
$$

or explicitely

$$
S_{g h}^{k L}=\left\{\begin{array}{cl}
C_{g h}^{k L} & \text { for } g \neq h \\
-\sum_{h^{\prime}(\neq g)} C_{g h^{\prime}}^{k L} & \text { for } g=h
\end{array}\right.
$$

From this we conclude

$$
\sum_{h} S_{g h}^{k L}=0 \quad \text { for each } g \text {. }
$$

This is another form of the acoustical condition.

Moreover interchange of $g$ with $h$ does not change the matrix elements :

$$
S_{g h}^{k L}=S_{h g}^{k L}
$$


We now consider how the submatrices $S_{g h}$ are mutually connected. For $g=h$ we can use again the matrices (13), which transform from one sublattice to another one:

$$
S_{h h}=R^{-1}(g \rightarrow h) S_{g g} R(g \rightarrow h)
$$

For $g \neq h$ we use the threefold symmetry of the body diagonals $\underline{d}_{g}$. E.g. a $120^{\circ}$-rotation around $\underline{d}_{1}=\frac{1}{\sqrt{3}}(1,1,1)$, which is described by a matrix

$$
u_{1}\left(\frac{2 \pi}{3}\right)=\left(\begin{array}{lll}
0 & 1 & 0 \\
0 & 0 & 1 \\
1 & 0 & 0
\end{array}\right)
$$

leaves the sublattice 1 invariant, but Inerchanges the other sublattices in the following manner:

$2 \rightarrow 3 \rightarrow 4 \rightarrow 2$. Thus

$$
S_{13}=u_{1}^{-1} S_{12} u_{1}
$$

and

$$
S_{14}=u_{1} S_{12} u_{1}^{-1}
$$

Similarly $120^{\circ}$-rotations around the other $\underline{d}_{g}$ can be used. The quantities $\varepsilon=\varepsilon_{g}^{k k}=-2 \sum_{h} C_{g h}^{k k}$ and $C_{g q}^{k k}$ do not depend on $g$. Hence after some calculations we get the following relations: 


$$
\begin{aligned}
& C_{34}^{k k}=C_{12}^{k k} \\
& C_{24}^{k k}=C_{13}^{k k} \\
& C_{23}^{k k}=C_{14}^{k k}
\end{aligned}
$$

Because of $(20)$ exactly the same relations hold for $S_{g h}^{k k}$. Most of the elements $C_{g h}^{K L}=S_{g h}^{k L}$ with $g \neq h$ and $K \neq L$ vanish. At the end of the last chapter we mentioned that

$$
C_{12}^{x y}=C_{12}^{x z}=0
$$

The other vanishing elements can be obtained by transformations like $(26,27)$.

Collecting the properties (11) - (15) and (18) - (29) we can finally write down $S_{g h}^{k L}$.

$$
S=\left(\begin{array}{ccc}
S^{x x} & S^{x y} & S^{x z} \\
& S^{y y} & S^{y z} \\
& & S^{z z}
\end{array}\right)
$$




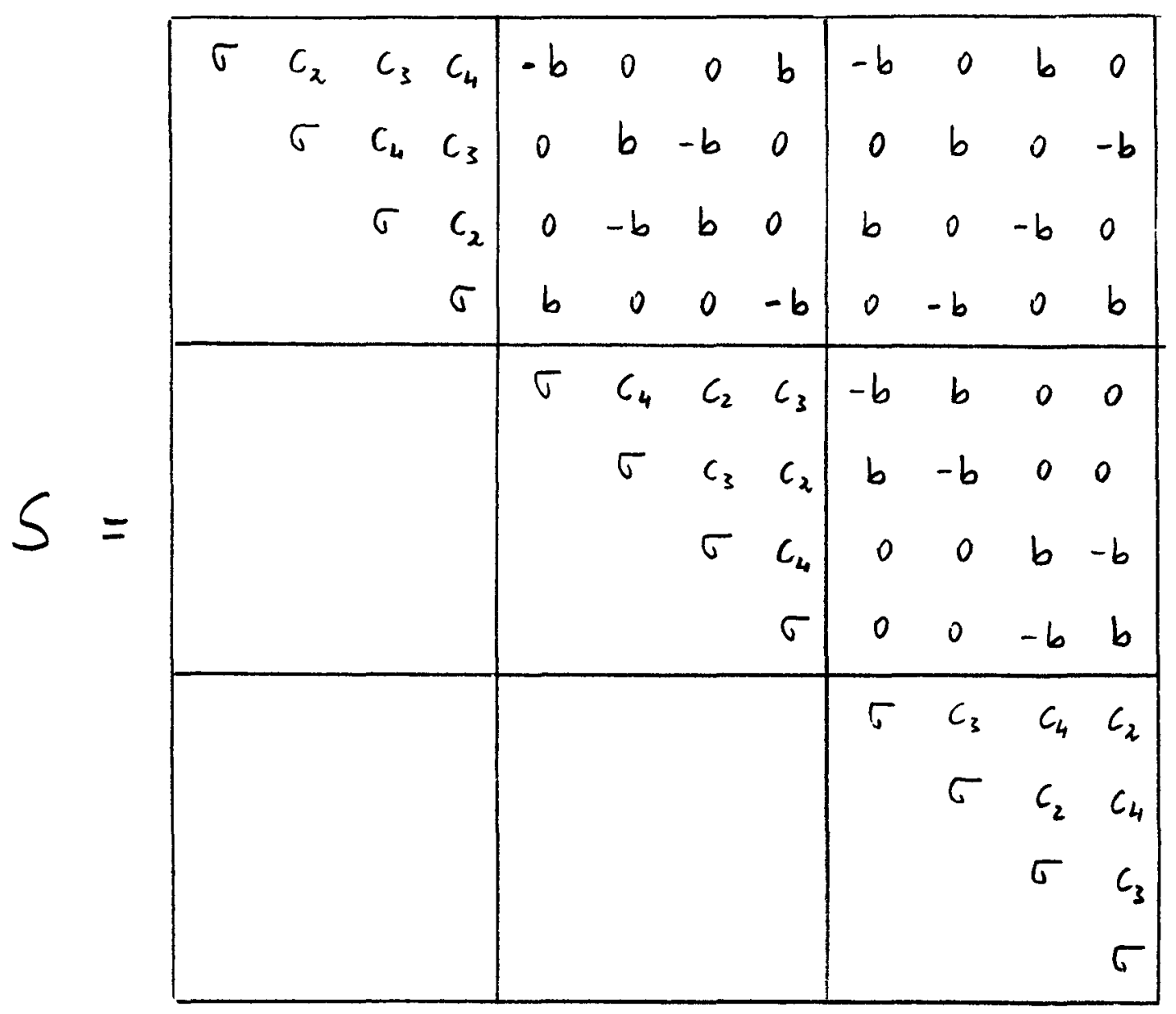

With the abbreviations

$$
\begin{aligned}
& \sigma=-c_{2}-c_{3}-c_{4} \\
& c_{g}=c_{1 g}^{x x} \\
& b=c_{14}^{x y}
\end{aligned}
$$

The matrix $D$ is obtained by $(15,15 a)$.

We see that only six constants must be calculated altogether: $c_{2}, c_{3}, c_{4}, b$ for $S$ and $\delta, \alpha$ or $\varepsilon, e$ for $D$. Nevertheless we have calculated all matrix elements $\left\langle k 0\left|W_{i j}\right| O L\right\rangle$ and all elements of $S$ and $D$ in order to test the numerical procedure by the symmetries and the vanishing elements of $S$ and $D$. 
The secular equation

$$
\operatorname{det}\left(S-\omega^{2} D\right)=0
$$

can be solved analytically applying the rules for determinants and yields the eigenvalues:

$$
\begin{array}{rlrl}
\omega_{1}^{2} & =0 & \text { threefold } \\
\omega_{2}^{2} & =\frac{-2 c_{3}-2 c_{4}+2 b}{\delta-\alpha} \quad \text { twofold } \\
\omega_{3}^{2} & =\frac{-2 c_{3}-2 c_{4}-4 b}{\delta+2 \alpha} \quad \text { single } \\
\omega_{4 / 5}^{2}=\frac{1}{\mu \nu}\left\{\frac{\nu \beta+\alpha}{2} \pm \sqrt{\left(\frac{v \beta-\alpha}{2}\right)^{2}+\nu\left(c_{3}-c_{4}\right)^{2}}\right\} \text { threefold each } \\
\text { with } \\
\alpha=-2 c_{2}-c_{3}-c_{4}-2 b \\
\Delta=-2 c_{2}-c_{3}-c_{4}+2 b \\
\mu & =\delta-\alpha \\
\nu & =1+2 \frac{\alpha}{\delta}
\end{array}
$$

In order to see the effect of the orientational order in $0-\mathrm{H}_{2}$ (fcc) and $p-D_{2}(f c c)$ let us consider the hypothetical $p-H_{2}$ (fcc) and $0-D_{2}$ (fcc) for a moment. Here the interaction is purely isotropic. Thus the single particle excitation energies are degenerate: $\varepsilon_{x}=\varepsilon_{y}=\varepsilon_{z}=\varepsilon$. The matrices $\varepsilon_{g}^{k L}$ and $D$ are diagonal: 


$$
\varepsilon_{g}^{k L}=\varepsilon \cdot \delta^{k L} \quad ; \quad D_{g h}^{k L}=\frac{1}{2 \varepsilon} \delta_{g h}^{k L}
$$

$S$ decays into the submatrices $S^{x x}, S^{y y}, S^{z z}$, which have all the same eigenvalues $\lambda_{1}=0 ; \lambda_{2}=-2\left(c_{2}+c_{3}\right) ; \lambda_{3}=-2\left(c_{2}+c_{4}\right)$; $\lambda_{4}=-\lambda\left(c_{3}+c_{4}\right)$. Two of them are degenerate because of $c_{2}=c_{4}$. or $C_{12}^{x x}=C_{14}^{x x}$. This relation holds, because the bonds connecting the sublattices 1 and 2 are transformed to bonds connecting 1 and 4 by a $90^{\circ}$-rotation around the $x$-axis. Finally we obtain the following eigenvalues for $p \cdot H_{2}(f c c)$ and $0-D_{2}$ (fcc):

$$
\begin{aligned}
\omega_{1}^{2} & =0 & & \text { threefold } \\
\omega_{2}^{2}=W_{3}^{2} & =W_{5}^{2}=-4 \varepsilon\left(c_{2}+c_{4}\right) & & \text { sixfold } \\
W_{4}^{2} & =-8 \varepsilon c_{2} & & \text { threefold }
\end{aligned}
$$

This must be compared with (33). We see that the orientational order causes a splitting of the sixfold degenerate optical line into three lines, whereas the threefold degenerate optical line $W_{4}$ is only shifted.

We have calculated the phonon energies for $\underline{k}=0$ in the realistic case of hexagonal $p-H_{2}$ and $0-D_{2}$ too. Using the results on page 51 we can specialize $(20,21)$ : 


$$
S_{g L}^{k L}=\left\{\begin{array}{cc}
\delta^{k L} C_{12}^{k k} & \text { for } g \neq h \\
-\delta^{k L} C_{12}^{k k} & \text { for } g=h
\end{array}\right.
$$

and (34):

$$
D_{g h}^{k L}=\frac{1}{2 \varepsilon_{k}} \delta_{g l}^{k l}
$$

with $\varepsilon_{x}=\varepsilon_{y}$ and $C_{12}^{x x}=C_{12}^{y}$. Then we get

$$
\begin{array}{ll}
W_{1}^{2}=0 & \text { threefold } \\
W_{2}^{2}=-4 \varepsilon_{x} C_{12}^{x x} & \text { twofold } \\
W_{3}^{2}=-4 \varepsilon_{z} C_{12}^{z z} & \text { single }
\end{array}
$$

The numerical results of $(33,35,38)$ are shown in table 3 ; they are given only for one value of $\alpha$ for each crystal type. A different choice of $\alpha$ within the range, which has been determined in IV, changes the energies at most by 1.58 .

Moreover the experimental results are cited in table 3, together with the results of other theories which have been worked out for $p-H_{2}$ and $0-D_{2}$. 
Table 3 Optical phonon frequencies

\begin{tabular}{|c|c|c|c|c|}
\hline crystal & $\begin{array}{l}\text { This work } \\
\text { with } V_{1}(+) \equiv 0 \\
\omega[\mathrm{meV}]\end{array}$ & $\begin{array}{c}\text { other theories } \\
\omega[\mathrm{mev}]\end{array}$ & $\begin{array}{l}\text { Neutron } \\
\text { scattering } \\
\omega[\mathrm{mev}]\end{array}$ & $\begin{array}{l}\text { Raman } \\
\text { scattering } \\
\omega[\mathrm{mev}]\end{array}$ \\
\hline $\mathrm{o}-\mathrm{H}_{2}(\mathrm{fcc})$ & $\begin{array}{l}9.78(2) \\
9.81(3) \\
10.28(1) \\
14.31 \quad(3) \\
(\alpha=0.13)\end{array}$ & & & $\begin{array}{r}7.7 \pm 0.01 \\
9.92 \pm 0.06 \\
11.52 \pm 0.12 \\
{[26]}\end{array}$ \\
\hline$p-D_{2}(f c c)$ & $\begin{array}{r}8.72(2) \\
8.74(3) \\
9.14(1) \\
12.79(3) \\
(\alpha=0.09)\end{array}$ & & & $\begin{array}{r}7.12 \pm 0.01 \\
9.24 \pm 0.06 \\
10.55 \pm 0.12 \\
{[26]}\end{array}$ \\
\hline $\begin{array}{l}\mathrm{p}-\mathrm{H}_{2} \text { (fcc) } \\
\text { hypothetic }\end{array}$ & $\begin{array}{l}9.36(6) \\
13.83 \quad(3) \\
(\alpha=0.10)\end{array}$ & $\begin{array}{c}7.93(6) \\
10.28(3) \\
{[27]} \\
\end{array}$ & & \\
\hline $\begin{array}{l}\text { o- } D_{2}(f C c) \\
\text { hypothetic }\end{array}$ & $\begin{array}{l}8.38(6) \\
12.16(3) \\
(\alpha=0.11)\end{array}$ & $\begin{array}{c}6.57(6) \\
9.1(3) \\
{[27]} \\
* \\
\end{array}$ & & \\
\hline$p-H_{2}(h c p)$ & $\begin{array}{l}6.0(2) \\
13.7(1) \\
1 \alpha=0.10\end{array}$ & $\begin{array}{c}5.02(2) \\
14.62(1) \\
{[47]} \\
*\end{array}$ & $\begin{array}{l}10.9 \\
{[35]}\end{array}$ & $\begin{array}{c}4.73 \pm 0.12 \\
-[21]\end{array}$ \\
\hline $0-D_{2}\left(h_{c p}\right)$ & $\begin{array}{l}5.6(2) \\
12.13(1) \\
(\alpha=0.11\end{array}$ & $\begin{array}{c}4.37(2) \\
13.45(1) \\
{[47]} \\
*\end{array}$ & $\begin{array}{l}4.6 \pm 0.1 \\
9.9 \pm 0.3 \\
{[36]}\end{array}$ & $\begin{array}{r}4.70 \pm 0.12 \\
- \\
{[21]}\end{array}$ \\
\hline
\end{tabular}

* one neighbour shell only 
In chapter III we have mentioned already that the distance dependent part $V_{1}(r)$ of the anisotropic van der Waals and overlap forces is not well known. In table 3 the results for $V_{1}(r) \equiv 0$ are given. The case $V_{1}(r) \neq 0$ will be discussed later.

\section{Comparison with the experiments}

Let us begin with the discussion of the splitting of the optical Iines in $0-H_{2}$ and $P-D_{2}$ due to the orientational order. Three lines have been observed [26] - We have calculated four lines; two of them are so close together that we have practically only three lines. However, the ratios of these lines differ from the measured ones. There are several possibilities which could explain this discrepancy:

1) If the ansotropic van der Waals and overlap forces (III, 1, 3) are included into the calculation, the splitting may become larger or smaller dependent on the choice of the parameters in $V_{1}(r)$ (See figure 3$)$. Because $V_{1}$ contains three free parameters $\left(\alpha^{\prime}\right.$, $\left(\beta_{1}\left(\beta_{2}\right)\right.$, there would be no problem to fit the calculated ratios to the observed ones. On the other hand the effect of $V_{1}(r)$ on the phonon energies is rather small $(<1 \%)$, if we use recent estimates of $V_{A}(r)[48]$.

2) The simple product ansatz $\psi_{i}^{0}=\varphi^{0}\left(\underline{R}_{i}\right) \cdot Y_{1,0}\left(R_{i}\right)(I, 7)$ for the one particle wavefunction is not exact. The exact ansatz $\psi_{i}^{0}=\sum_{m}^{\nabla} \varphi^{m} Y_{n, m}(I, 2)$ would give another splitting. We assume the difference to be small however, since the rigid lattice libron theories have been successfull. See the detailed discussion in $(I, 2-8)$.

3) An obvious lack of the theory is the use of an isotropic Gaussian for $\varphi_{i}^{0}$, which does not reflect the point symmetry of 
the $\mathrm{Pa} 3$ space group. (See $\mathrm{V}, 60,61)$. The self consistent determination of $\varphi_{i}{ }^{0}$ using an anisotropic $\varphi_{i}{ }^{0}$ like $(I I, 20)$ in the ground state variation would be a considerable improvement. The explicite calculation of the matrix elements would however be very complicated.

Next we have to discuss the absolute values of the phonon energies. We see that our results must be multiplied by a nearly constant factor (about 0.8 for $H_{2}$ and 0.83 for $D_{2}$ ) in order to obtain the experimental results. This factor cannot be explained by the points 1) - 3), which concern the splitting. We must think of two other approximations which have been made in our theory:

4) It must be tested, whether it is justified to treat not only the ground state but also the lowest excited states of the quantum crystals $H_{2}$ and $D_{2}$ formally by the harmonic theory using the effective interaction $W_{i j}(V I, 13)$ instead of the true interaction $V_{i j}(I I I, 1)$. This question has been discussed already in detail in chapter $V$. We have seen that we must compare $(v, 51)$ with $(v, 52)$ in order to get a test. The comparison can be restricted to the diagonal elements $\varepsilon_{g}^{k k}$, because the absolute values of the phonon energies are not much influenced by the off-diagonal elements. We define

$$
\Delta=\frac{\varepsilon_{E}-\varepsilon_{C P}}{\varepsilon_{E}}
$$

$\varepsilon_{C P}(V, 51)$ is calculated by the coupling parameters, $\varepsilon_{E}$ $(v, 52)$ is calculated as the sum of the kinetic and the potential excitation energies. The results are listed in table 4.

$\triangle$ measures the uncertainties of our results due to the deviations of the molecular field from an harmonic oscillator potential. We see that $\Delta$ is to small to explain the above mentioned factors 0.8 and 0.83 . 
Table 4

\begin{tabular}{|c|c|c|c|c|}
\hline crystal & $\alpha$ & $\begin{array}{r}\varepsilon_{E} \\
{[\mathrm{meV}]}\end{array}$ & $\begin{array}{c}\varepsilon_{c p} \\
{[\mathrm{meV}]}\end{array}$ & $\Delta_{[8]}$ \\
\hline $\begin{array}{l}0-\mathrm{H}_{2} \\
(f c c)\end{array}$ & $\begin{array}{l}0.10 \\
0.13\end{array}$ & $\begin{array}{l}9.59 \\
9.96\end{array}$ & $\begin{array}{l}9.99 \\
9.90\end{array}$ & $\begin{array}{l}-4.28 \\
+0.68\end{array}$ \\
\hline $\begin{array}{l}p-D_{2} \\
(f c c)\end{array}$ & $\begin{array}{l}0.07 \\
0.09 \\
0.11\end{array}$ & $\begin{array}{l}8.76 \\
8.93 \\
9.08\end{array}$ & $\begin{array}{l}8.94 \\
8.84 \\
8.77\end{array}$ & $\begin{array}{l}-28 \\
+18 \\
+3.48\end{array}$ \\
\hline $\mathrm{p}-\mathrm{H}_{2}$ & 0.10 & 8.70 & 9.45 & -8.68 \\
\hline$O-D_{2}$ & $\begin{array}{l}0.09 \\
0.11\end{array}$ & $\begin{array}{l}8.52 \\
8.66\end{array}$ & $\begin{array}{l}8.53 \\
8.41\end{array}$ & $\begin{array}{l}-0.028 \\
+2.98\end{array}$ \\
\hline $\begin{array}{l}p-H_{2} \\
O-D_{2}\end{array}$ & $\begin{array}{l}\text { Nosanow's } \\
\text { SRC-funct. }\end{array}$ & $\begin{array}{l}8.34 \\
7.77 \\
\end{array}$ & $\begin{array}{l}9.65 \\
9.02 \\
\end{array}$ & $\begin{array}{r}-15.78 \\
-16.18 \\
\end{array}$ \\
\hline
\end{tabular}

In the lower part of table 4 a comparison is made with the results obtained with Nosanow's SRC-function (II, 14). $\Delta$ is rather large here, probably because the one parameter function (II,14) is not flexible enough to be used for the ground state and for the excited states.

5) We discuss now the most severe approximation which has been made in this work. In the RPA we have neglected all anharmonic terms,

These cause a damping and a shifting of the phonon energies. Looking at the results of Horner [46] for solid helium we are allowed to expect that especially the shifting is a large effect also in $H_{2}$ and $D_{2}$ and can amount to a factor of about 0.8 . The inclusion of the anharmonic terms into the theory is planned for 
the future work.

The last point in this chapter is the comparison with other theories. For the disordered hcp phase of $H_{2}$ and $D_{2}$ a nearest neighbour self consistent phonon approximation (collective plcture) has been used [47]. The low optical line agrees well with the experiments, but the energy of the high mode is much to large (358).

The hypothetic $p-H_{2}(f c c)$ and $O-D_{2}$ (fcc) has been treated in the one particle picture, which is used in this work too [27]. Only nearest neighbour interaction is considered and Nosanow's SRCfunction is used. As to the ground state the results seem to agree with ours. But the optical phonon frequencies are lower and thus better than ours. However the difficulties due to the discrepancy between the two values for $\varepsilon$ have not been discussed. Looking at the high value of $\Delta$ which we obtained with Nosanow's function, the phonon energies in $[27]$ seem to be uncertain within about $16 \%$.

\section{Dispersion curves and spectrum of the phonons}

For $\underline{k} \neq 0$ the secular equation cannot be solved analytically. In this work for the orientationally ordered crystals $\mathrm{O}-\mathrm{H}_{2}$ and $p-D_{2}$ the numerical solution is carried out only for the case of phonons.

The librons in these crystals have been treated already in [18, 19]. The eigenvalue problem for the librons

$$
\sum_{A, h} S_{g h}^{\alpha \beta} C_{h}^{\beta}=\frac{w^{2}}{2 \varepsilon_{\alpha}} C_{g}^{\alpha} ; \quad\left(\varepsilon_{\alpha}=\varepsilon_{\bar{\alpha}}\right)
$$


has been solved numerically by the method of Jacobi [49]. In this method the hermitian secular matrix $S$ is diagonalized iteratively by asequence of two-dimensional unitary transformations. Details can be found e.g. In [19].

For the phonons in the ordered crystals we have to solve the generalized eigenvalue problem

$$
\sum_{L, h} S_{g h}^{k L} C_{h}^{L}=\omega^{2} \sum_{L, h} D_{g h}^{k L} C_{h}^{L}
$$

Here we use a generalization of Jacobi's method [50]. Since the eigenvectors $C$ of (2) are not orthogonal, the transformations which are used for the iteration, are no longer unitary.

In the figures 4 and 5 dispersion curves of the phonons in $0-H_{2}$ and $p-D_{2}$ are shown. In the directions of the body diagonals, which are axes of threefold symmetry, the splitting of the curves due to the orientational order is rather large but restricted to two optical Iines. As a comparison the dispersion curves of $p-H_{2}$ for these directions are shown too.

In direction of low symmetry (e.g. the cubic axes) the degeneracies of all lines are lifted but the splittings are smaller than those in the directions of symmetry.

All results are given for the case $V_{1}(r) \equiv 0$, since the influence of $V_{1}$ on the phonons seems to be small (VIII, point 1 ).

The figures 6 and 7 show the phonon spectrum of $0-H_{2}$ and of the hypothetic $\mathrm{p}-\mathrm{H}_{2}$ for comparison. We see that the effect of the orientational order cannot be observed in the resolution of $0.2 \mathrm{mev}$, which we have reached by the computation of the eigenvalues for 1782 points of $\underline{h}$-space.

The lattice dynamics of the hcp phases has been trated too. In 
the figure 8 the calculated dispersion curves for $0^{-D_{2}}$ are compared with recent neutron diffraction experiments [36]. Because of reasons, which have been discussed in (VIII, point 4,5), our energies are too large. The factor is nearly the same $(\sim 1.20)$ for all modes and for all points of the Brioullin zone.

The calculated phonon spectrum of $\mathrm{p}^{-\mathrm{H}_{2}}$ is compared in the figure 9 with a measured spectrum of $\mathrm{H}_{2}$ containing $6880-\mathrm{H}_{2}[35]$. The experiment was performed at $4.3^{\circ} \mathrm{K}$ within the disordered hcp-phase. Thus we are allowed to compare with our $\mathrm{p}-\mathrm{H}_{2}$ spectrum. The shape of the two spectra is nearly the same, apart from the above mentioned factor.

We can get a first approximation for the long range correlation energy (see end of part B) by compairing the single particle excitation energies $\varepsilon$ with the first moment $\bar{w}$ (center of gravity) of the spectra. The effective Hamiltonian diagonalized in the RPA has the form

$$
X=E_{0}-\frac{1}{\lambda}\left(N \varepsilon-\sum_{k, \lambda} \omega_{k}^{\lambda}\right)+\sum_{k, \lambda} \omega_{k}^{\lambda} B_{k}^{\lambda+} B_{k}^{\lambda}
$$

( $B_{k}^{\lambda+}, B_{k}^{\lambda}$ create resp. destruct a phonon with wave vector $\underline{k}$ and polarisation $\lambda$ ).

$\varepsilon-\bar{\omega}$ is very close to $0.4 \mathrm{meV}$ for all isotopes and lattice structures considered. Thus the Hartree ground state energies $E_{0} / N_{\text {, }}$ which are listed in table 1 , are lowered by about $0.2 \mathrm{meV}$ by the long range correlations.

The secular problem

$$
\sum_{B, h} S_{g h}^{A B} C_{h}^{B}=w^{2} \sum_{B, h} D_{g h}^{A B} C_{h}^{B}
$$

for the coupled system of phonons and librons will be solved in a second paper. The matrix elements of $\left[W_{i j}\right]$, which we shall need to calculate the elements $S_{g h}^{k \alpha}$ and $S_{g h}^{\alpha k}$ are already known from chapter VII. Therefore only a considerable amount of numerical work has to be done. For the diagonalization of (4) another generalization of the Jacobi method must be used, because the complex matrix $S$ is normal but not hermitian (see page 62). 
It is a pleasure for me to thank Prof. G. Leibfried for his interest and Dr. W. Biem for his constant advice and help.

This work has been supported by the ministery for scientific research of the Federal Republic of Germany under the grant number PF 4.004 .

I owe my participation on the "Conference on Quantum Crystals" in Aspen (Colorado), USA (1969) to the ministery of scientific research and to the FAHO (Gesellschaft der Freunde der Aachener Hochschule). 
$-94-$

DA pe nd ix

$\underline{x}$ Series expansion of integrals

We consider the integral

$$
]=\sqrt{\frac{r}{2 \pi}} \int_{0}^{\infty}\left[e^{-\frac{r}{2}\left(t-r_{0}\right)^{2}}-e^{-\frac{r}{2}\left(r+r_{0}\right)^{2}}\right] f^{2}(r) \bar{v}_{0}(r) \frac{r}{r_{0}} d r
$$

with

$$
\begin{aligned}
& V_{0}(r)=V_{0}(r)-\frac{\hbar^{2}}{2 M} \nabla^{2} \ln f(r) \\
& V_{0}(r)=4 \varepsilon\left[\left(\frac{5}{r}\right)^{12}-\left(\frac{5}{r}\right)^{6}\right] \\
& f(r)=(\pi, 14) \text { or }(\pi, 21)
\end{aligned}
$$

If $r_{0}$ is sufficiently large compared with the hard core parameter $\sigma$ and if the width of the Gaussians is mall compared with $r_{0}$ $\left(\gamma r_{0}^{2} \gg 1\right)$, we can replace (1) by

$$
I=V_{0}\left(r_{0}\right) \sqrt{\frac{r}{\lambda \pi}} \int_{t_{\min }}^{\infty} e^{-\frac{1}{2} \gamma\left(r-r_{0}\right)^{2}}\left(\frac{r_{0}}{r}\right)^{5} d r
$$

Since in (2) the hard core is no longer eliminated by the SRCfunction $f(r)$, the region near $r=0$ must be excluded from the integration. The integrand becomes very small between $t=0$ and $r=r_{0}$, thus we can choose the position of the minimum as lower limit of the integration: $t_{\min }\left(\ll r_{0}\right)$. (2) can be 
transformed:

$$
I=V_{0}\left(r_{0}\right) \sqrt{\frac{r}{2 \pi}} \int_{-r_{0}+r_{\min }}^{r_{0}-r_{\min }} e^{-\frac{1}{2} \gamma r^{2}} \frac{1}{\left(1+\frac{r}{r_{0}}\right)^{5}} d r
$$

Because the integrand is localized around $T=0$, we can take $r_{0}-r_{\mathrm{mim}}$ as upper integration limit. (3) can be expanded into a convergent series of integrals:

$$
]=V_{0}\left(r_{0}\right) \sqrt{\frac{r}{2 \pi}} \int_{-r_{0}+r_{\min }}^{r_{0}-r_{\min }} d r e^{-\frac{1}{2} r r^{2}}\left[1+\frac{5 \cdot 6}{2 !}\left(\frac{t}{r_{0}}\right)^{2}+\frac{5 \cdot 6 \cdot 7 \cdot 8}{4 !}\left(\frac{r}{r_{0}}\right)^{4}+\cdots\right]
$$

(The odd terms of the series vanish)

In order to make this series practicable, we use infinite integration limits and obtain

$$
\begin{aligned}
] \approx I_{n}=V_{0}\left(\gamma_{0}\right) & {\left[1+\frac{5 \cdot 6}{2} \frac{1}{\gamma r_{0}^{2}}+\frac{5 \cdot 6 \cdot 7 \cdot 8}{2 \cdot 4}\left(\frac{1}{\gamma r_{0}^{2}}\right)^{2}\right.} \\
& \left.+\cdots+\frac{5 \cdot 6 \cdot 7 \cdot \cdot(2 n+4)}{2 \cdot 4 \cdot 6 \cdots 2 n} \cdot\left(\frac{1}{\gamma r_{0}^{2}}\right)^{m}\right]
\end{aligned}
$$

(5) is the first part of a semi-convergent series. Th1s means that the elements of the series decrease for $\mu<M_{0}$ but increase for $m>n_{0}$. We can approximate $\left.J_{\text {by }}\right]_{n}$. if we choose $M<M_{0}$.

In the case of $H_{2}$ and $D_{2}$ it turns out that the conditions for 
$-96-$

using (2) Instead of (1) are fulfilled for $r_{0}>\sqrt{5}, R_{0}, n_{0}$ is larger than 100. An excellent approximation for $]$ is obtrained already by the first three terms of (5).

XI Special matrix elements

We need the matrix element

$$
\begin{aligned}
& \left\langle m_{i}\left|Y_{2, \mu}\left(\Omega_{i}^{r_{0}}\right)\right| m_{i}^{\prime}\right\rangle
\end{aligned}
$$

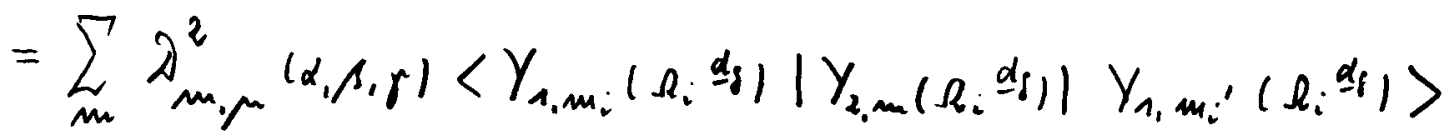

The Euler angles $\alpha, \beta, \gamma$ describe the rotation of the coordinate system with $\underline{d} g$ as z-axis to the system with $\underline{r}_{0}$ as $z$-axis. Using $[38,4.34]$ we get

$$
\begin{aligned}
& (1)=\sqrt{\frac{5}{4 \pi}} C\left(121_{i} 00\right) \cdot C\left(121_{i} m_{i}^{\prime}, m_{i}-m_{i}^{\prime}\right) \cdot \partial_{m_{i}-m_{i}^{\prime}, \mu}^{2}\left(\alpha_{1}, \mu_{1} \gamma\right) \\
& =-\sqrt{\frac{2}{5}} \sqrt{\frac{5}{4 \pi}} C\left(12 \Lambda_{i} m_{i}, m_{i}-m_{i}^{\prime}\right) \cdot \lambda_{m_{i}-m_{i}^{\prime}, \mu}^{2}(\alpha, \Lambda, \gamma)
\end{aligned}
$$

As a special case we consider $m_{i}=m_{i}^{\prime}=0$ : 
$-97-$

$$
\begin{aligned}
& \left\langle 0\left|Y_{2, \mu}\right| 0\right\rangle=\frac{2}{5} \sqrt{\frac{5}{4 i \pi}} \partial_{0, \mu}^{2}(0, \mu, \gamma) \\
& =\frac{2}{5} \sqrt{\frac{5}{4 \pi}} \lambda_{-\mu, 0}^{2 *}(-\gamma,-\mu, 0) 38,4.21 \\
& =\frac{2}{5} Y_{2, \mu}\left(-\beta_{1}-\gamma\right) \\
& =\frac{2}{5} Y_{2, \mu}\left(\Omega_{\underline{d}_{j}}^{\underline{\tau_{0}}}\right)
\end{aligned}
$$

$\Omega_{d_{j}}^{T_{0}}=$ polar angles of $\underline{d}_{g}$ with respect to $\underline{r}_{0}$. 
[1] O. Nagai and T. Nakamura

Progr. Theor. Phys. 24, 432 (1960); 30, 412 (Errata) (1963)

[2] J. Felsteiner,

Phys. Rev. Lett. 15, 1025 (1965)

[3] J.C. Raich and H.M. James

Phys. Rev. Lett. 16, 173 (1966)

[4] H.M. James and J.C. Raich

Phys. Rev. 162, 649 (1967)

[5] H.M. James

Phys. Rev. 167, 862 (1968)

[6] J.C. Raich and R.D. Etters Phys. Rev. 155, 457 (1967)

[7] R.L. Mills and A.F. Schuch

Phys. Rev. Letters 15, 722 (1965)

[8] A.F. Schuch and R.L. Mills Phys. Rev. Lett. 16, 616 (1966)

[9] A.F. Schuch, R.L. Mills and D.A. Depatie Phys. Rev. 165, 1032 (1968)

[10] K.F. Mucker, S. Talhouk, D.M. Harris, D. White and R.A. ErickPhys. Rev. Lett. 16, 799 (1966)

[11] K.F. Mucker, P.M. Harris, D. White and R.A. Erickson J. Chem. Phys. 49, 1922 (1968)

[12] R.W. Hill and B.W. Ricketson Ph11. Mag. 45, 277 (1954)

[13] G. Ahlers and W.H. Orttung Phys. Rev. 133, A 1642 (1964)

[14] G. Grenier and D. White

J. Chem. Phys. 40, 3015 (1964)

[15] S. Homma, K. Okada, and H. Matsuda Progr. Theor. Phys, 38, 767 (1967)

[16] H. Ueyama and T. Matsubara Progr. Theor. Phys. 38, 784 (1967)

[17] J.C. Raich and R.D. Etters Phys. Rev. 168, 425 (1968) 
[18] F.G. Mertens, W. Biem, and H. Hahn

Z. Physik 213, 33 (1968); 220, 1 (1969)

[19] F.G. Mertens

Report of the nuclear research center Jullich (Germany), Jül-564-FN (1968), unpublished

[20] W.N. Hardy, I.F. Silvera, and I.P. McTague

Phys. Rev. Lett. 22, 297 (1969)

[21] I.F. Silvera, W.N. Hardy, and I.P. McTague

Disc. Faraday Soc.,48, 54 (1969)

[22] A.J. Berlinsky, A.B. Harris, and C.F. Coll III

Sol. State Comm. $I, 1491$ (1969)

[23] A.B. Harris,

Phys. Rev. B 1,1881 (1970)

[24] C.F. Coll III, and A.B. Harris, and C.F. Coll III Phys. Rev. Lett. 25, 858 (1970)

[25] Stockmeyer and Stein

Nuclear research center Jülich (Germany), private communication

[26] W.N. Hardy, I.F. Silvera, K.N. Klump and O. Schnepp Phys. Rev. 21, 291 (1968)

[27] K.N. Klump, O. Schnepp, and L. Nosannw

Phys. Rev. B l, 2496 (1970)

[28] C.M. Cunningham, D.S. Chapin, and H.L. Johnston Journal Am. Chem. Soc. 80,2382 (1958)

[29] L.H. Nosanow,

Phys. Rev. L. 13, 270 (1964), Phys. Rev, 146, 120 (1966)

[30] W.J. Milllin,

Phys. Rev. 134, A 1249 (1964)

[31] L.H. Nosanow,

Proc. of the Ninth Int. Conf. on Low Temp. Phys..., Columbus, Ohio, USA (1964) Plenum Press, Inc., N.Y: (1965)

[32] J.H. Hetherington, W.J. Mullin, and L.H. Nosanow Phys. Rev. 154, 175 (1967)

[33] w. Brenig, z, Physik 171, 60 (1963)

[34] W. Blem, to be published

W. Blem, Report of the nuclear research center Jullch (Germany), Jul-496-NP (1967), unpublished 
[35] W. Schott

Z. Physik 231, 243 (1970)

[35a] W. Schott, private communication

[36] M. Nielson, H. Bjerrum Moller, and w. Meyer Bull. Am. Phys. Soc. 15,393 (1970); private communication

[37] J.A. Krumanansl and S.Y. Wu Physics Lett. 28 A, 263 (1968) S.Y. Wu Thesis, University of Philadelphia, Penns. (USA), unpublished

[38] M.E. Rose

Elementary Theory of Anqular Momentum, John Wiley and Sons New York 1957

[39] J.O. Hirschfelder, C.F. Curtiss, and R.B. Bird Molecular Theory of Gases and Liquids, J. Wiley and Sons, New York 1954

[40] T. Nakamura, Progr. Theor. Phys. 14, 135 (1955)

[41] R.J. Elliott and W.M. Hartmann Proc. Phys. Soc. 90, 671 (1966)

[42] H.P. Gush and J. van Kranendonk Can. J. Phys. 40, 1461 (1962)

[43] A. M. Michels, W. de Graaff, and C.A. ten Seldam Physica 26, 393 (1960)

[44] R.W. Hill and O.V. Loumasmaa Phil. Mag. 4, 785 (1959)

[45] P. Gillessen and W. Biem

z. Physik 216, 499 (1968)

[46] H. Horner, to be published

[47] M.L. Klein and T.R. Koehler Journ. of Phys. C, L 102 (1970)

[48] W.D. Davison

Disc. Faraday Soc. 33,71 (1962)

[49] C.G.J. Jacobi, J. reine u. angewandte Math. 30, 51 (1846)

[50] S. Falk, and P. Langemeyer Elektronische Datenverarbeitung S. 30 (1960) 


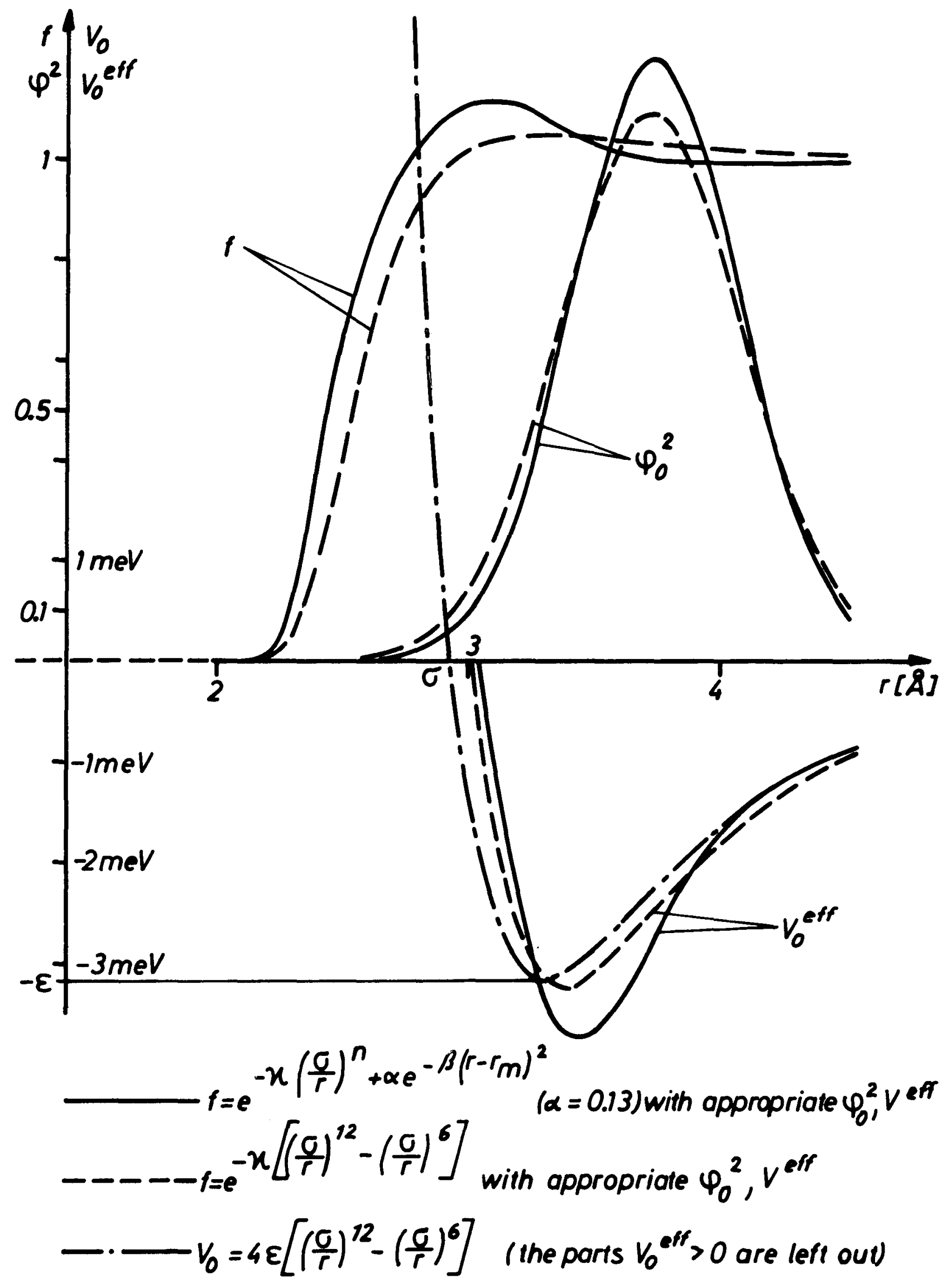

Figure1 SRC-function, 1-porticle-function; effective -potential for $\mathrm{O}-\mathrm{H}_{2}$ (ffc) 


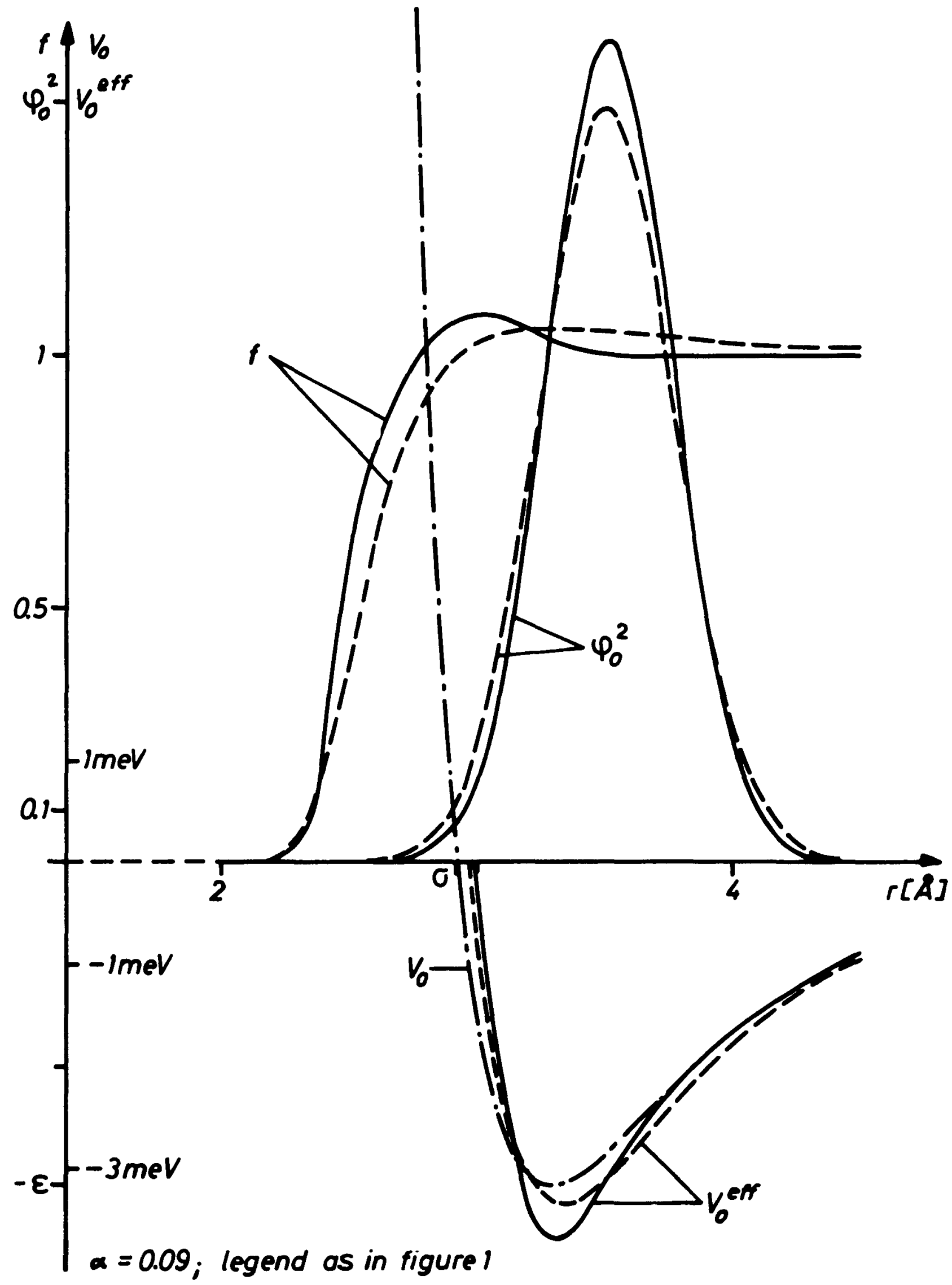

Figure 2 SRC-function, 1-particle-function, effective potential for $p-D_{2}$ (fcc) 


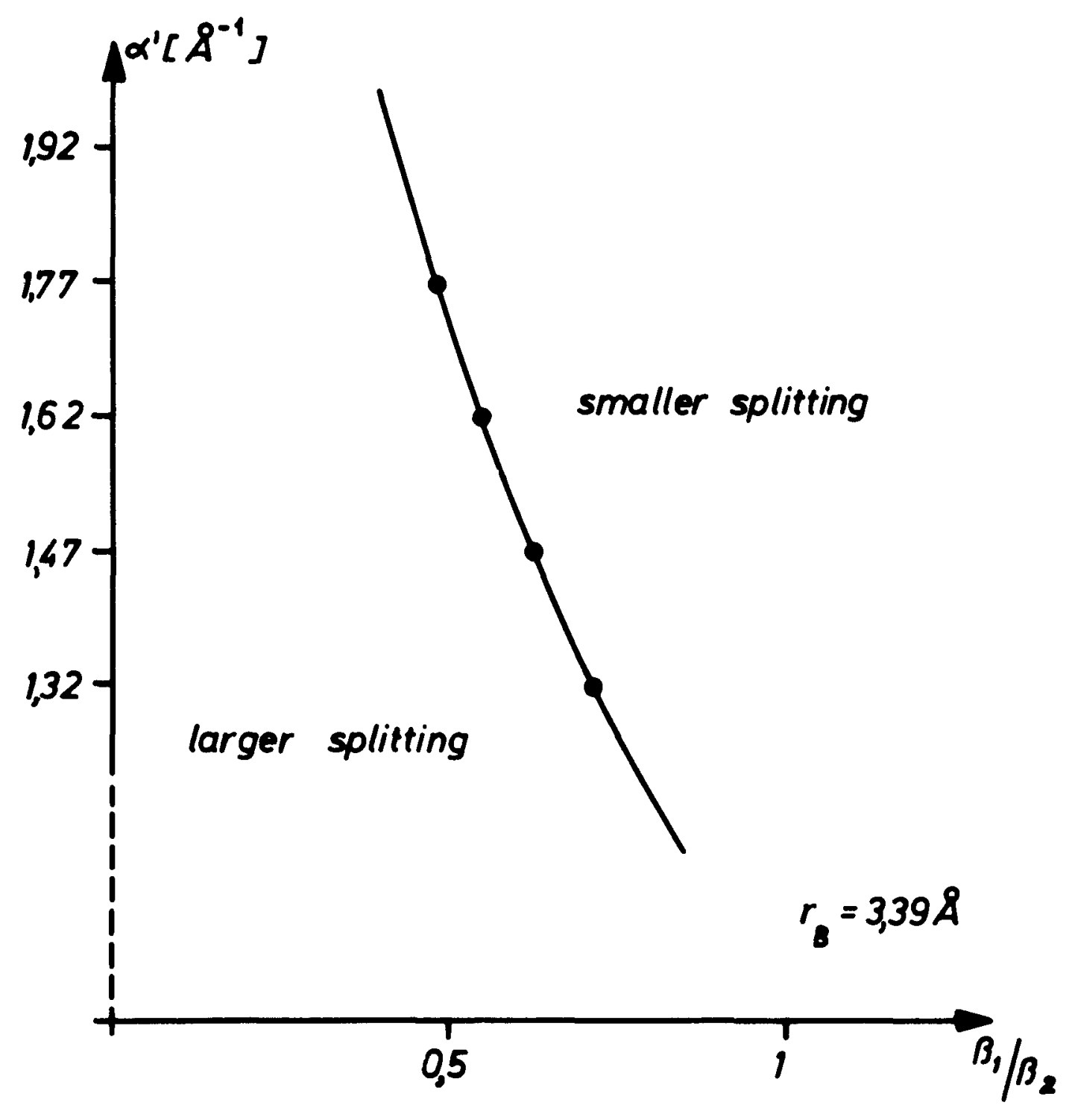

Effect of $V_{1}(r)=\beta_{1} e^{-2 \alpha^{\prime}\left(r-r_{B}\right)}-\beta_{e}\left(\frac{\left.r_{1}\right)^{6}}{r}\right.$

on the splitting of the optical phonon

frequencies in $\mathrm{O}-\mathrm{H}_{2}(f \subset C)$

compared with the splitting for $V_{1}(r) \equiv 0$

Fig. 3 


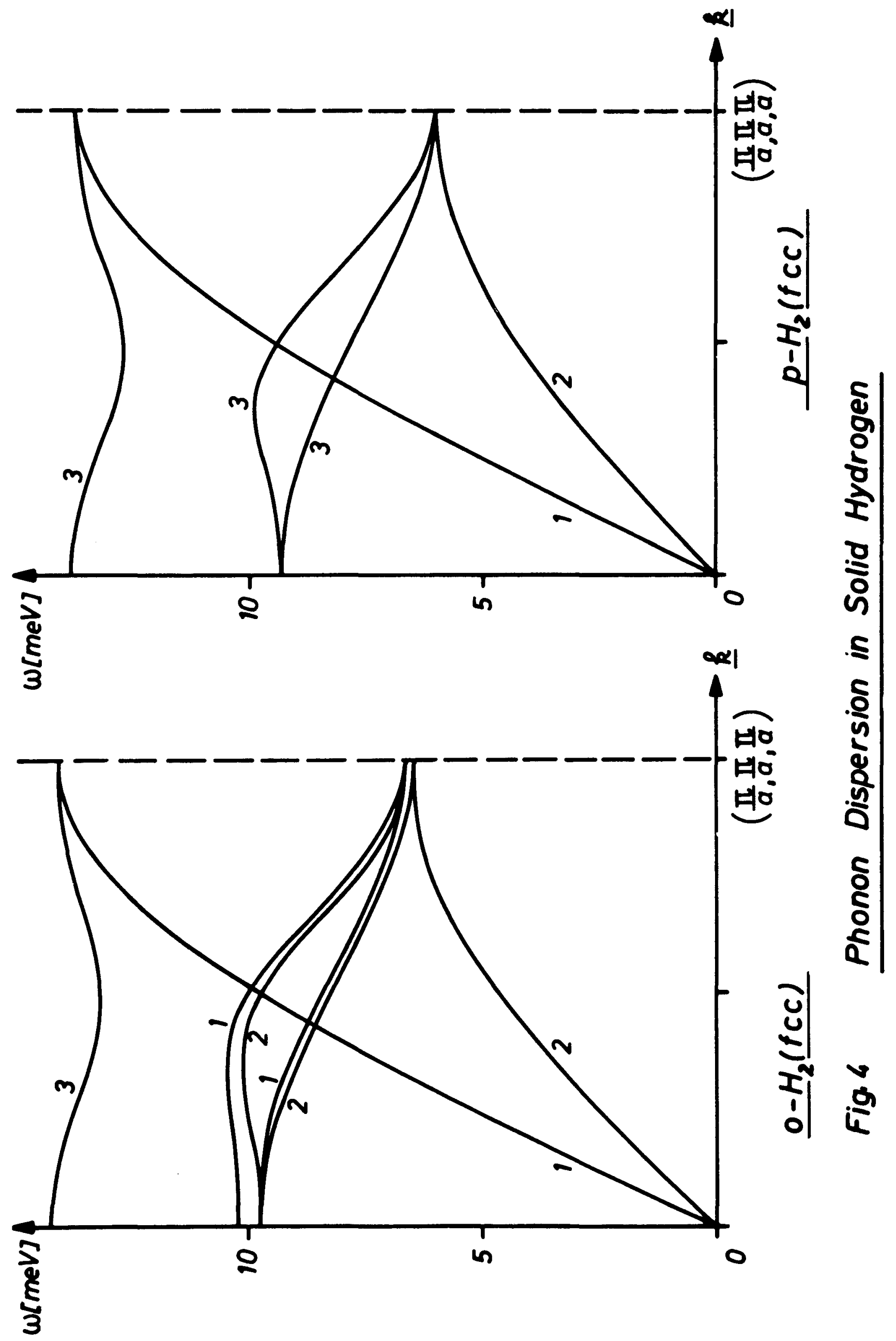




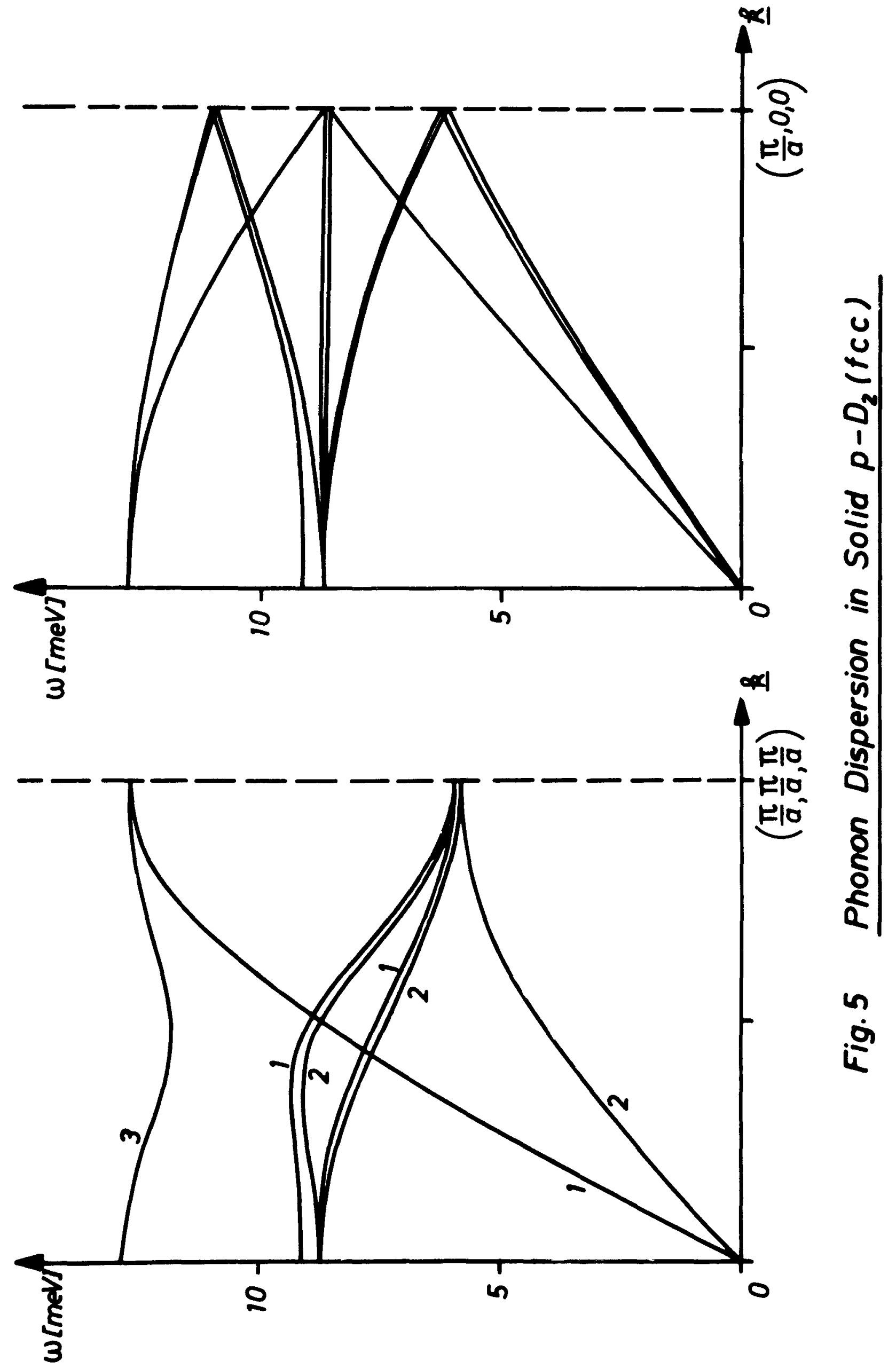




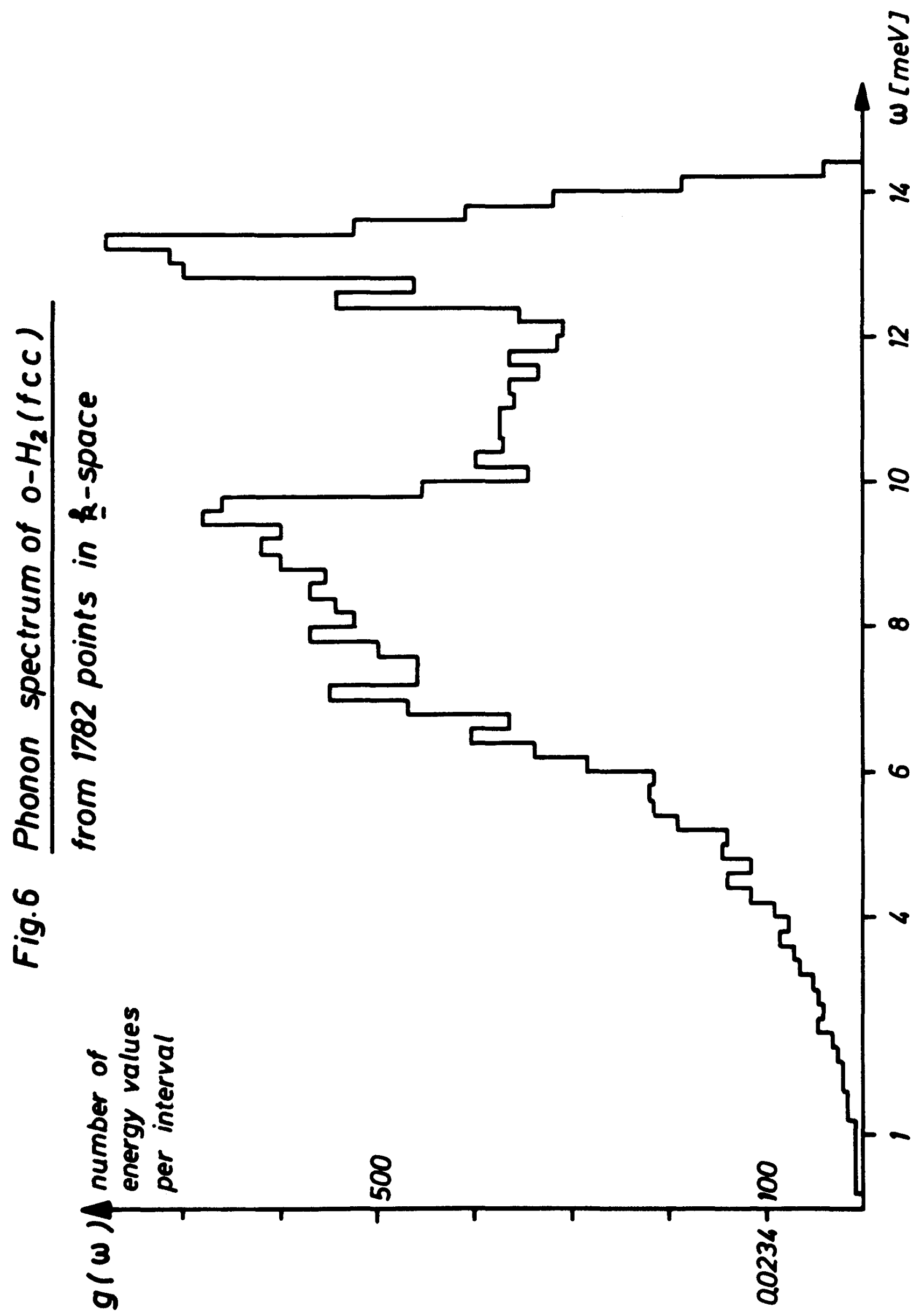




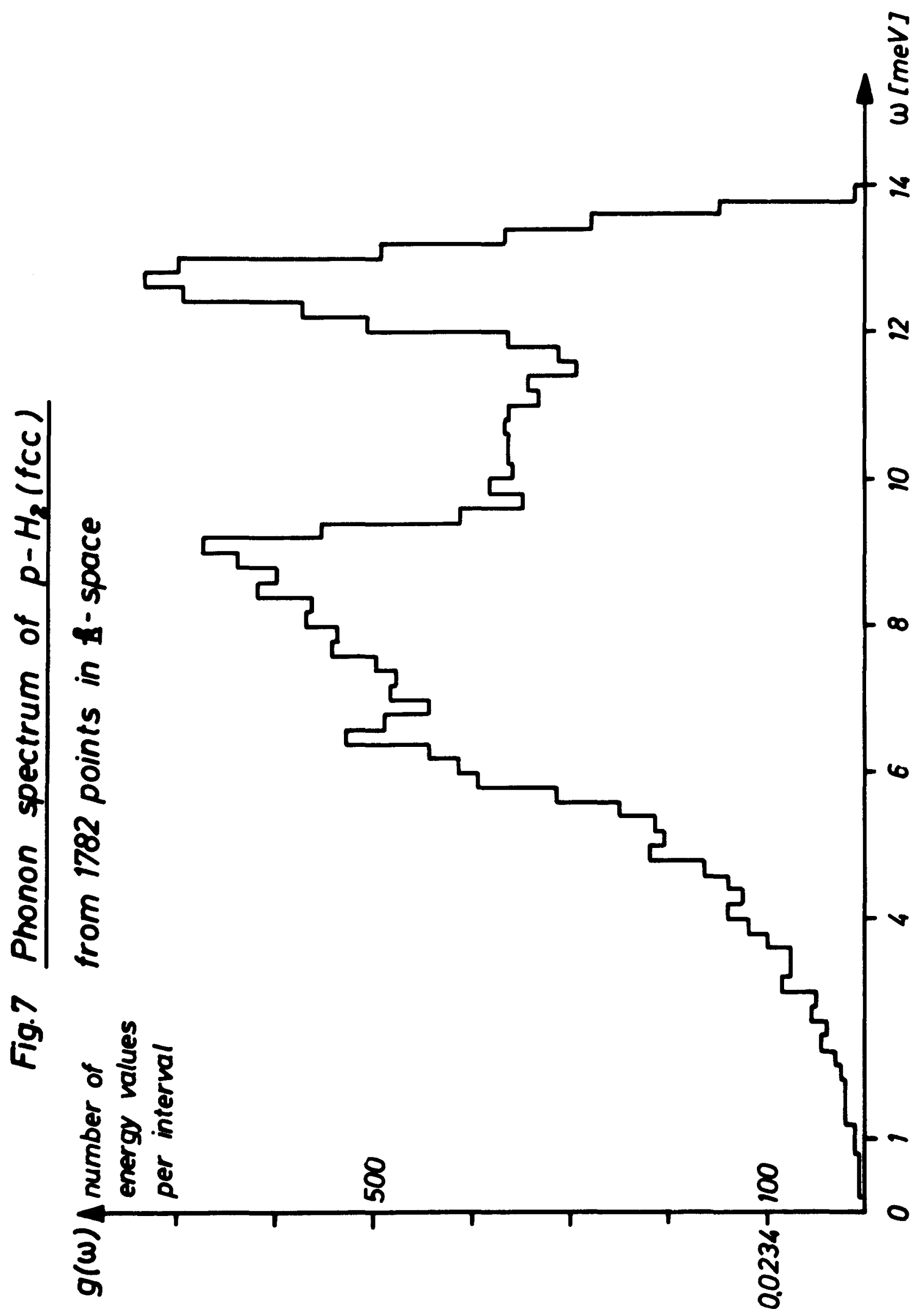


Fig.8 Phonon dispersion in solid $0-D$ (hcp)
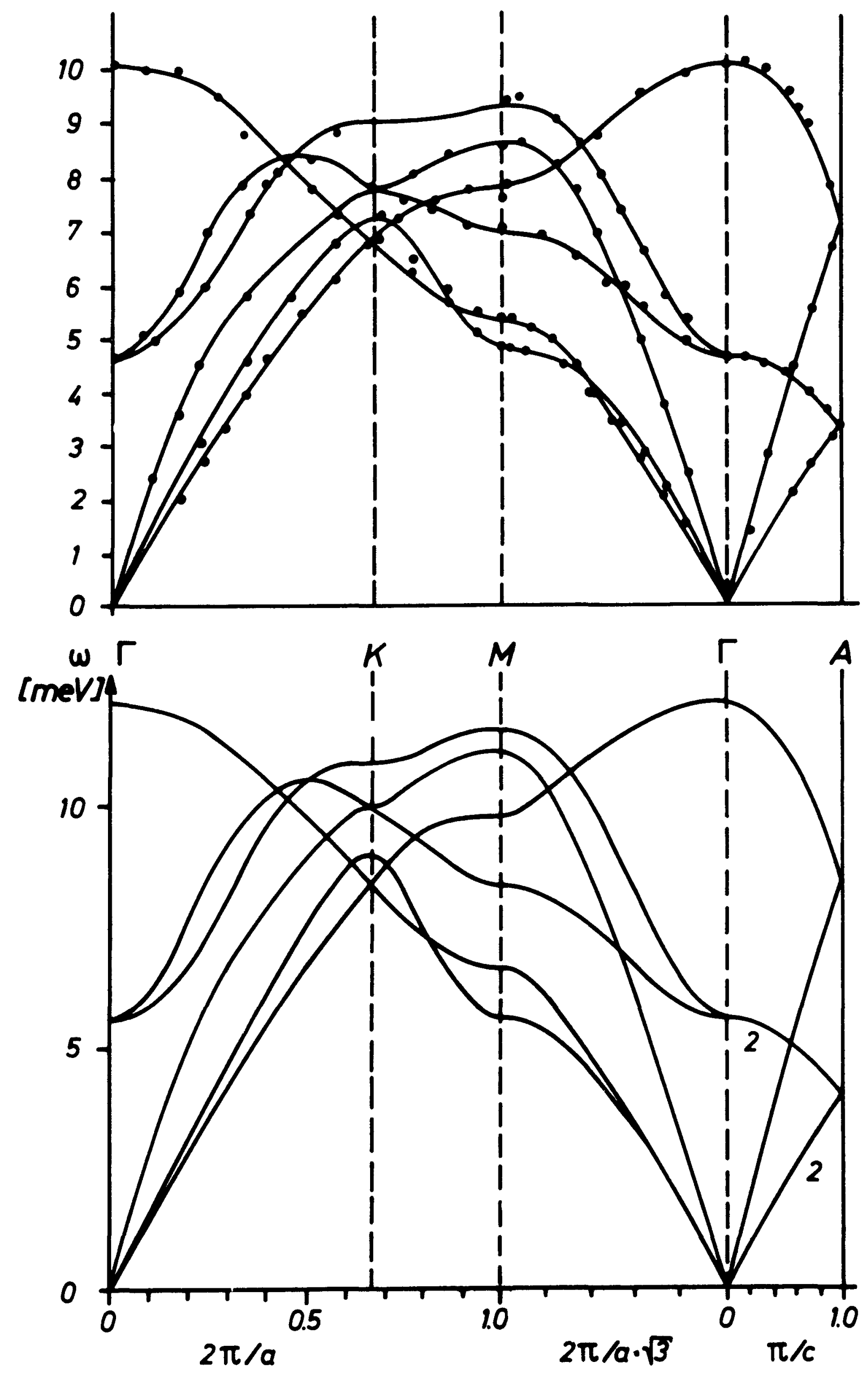
Fig.9 Phonon spectrum of solia hydrogen (hcp)

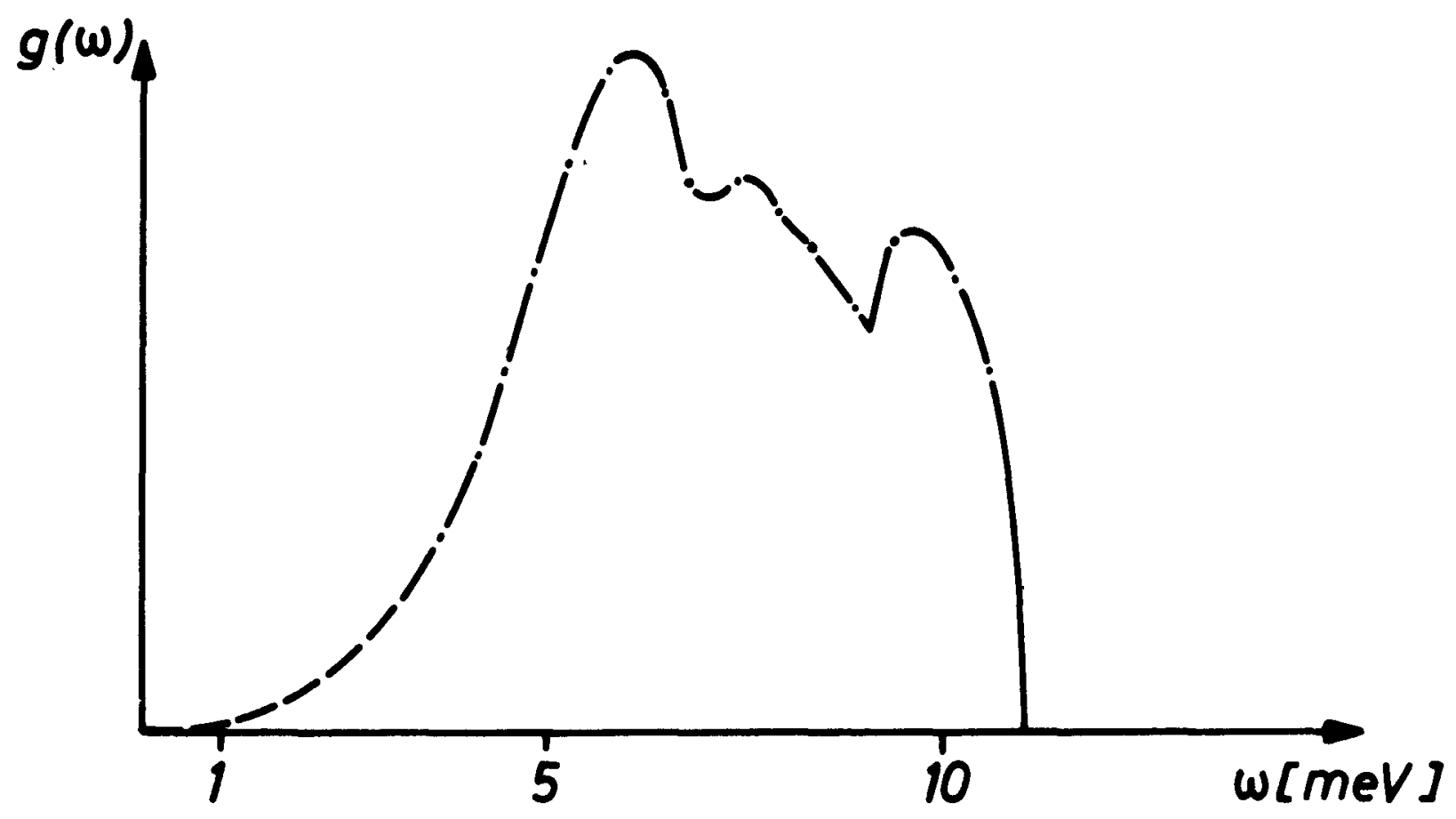

$68 \%$ o- $\mathrm{H}_{2}$ (hcp) $\quad \mathrm{T}=4.3 \circ \mathrm{K} \quad$ Expt. [35]

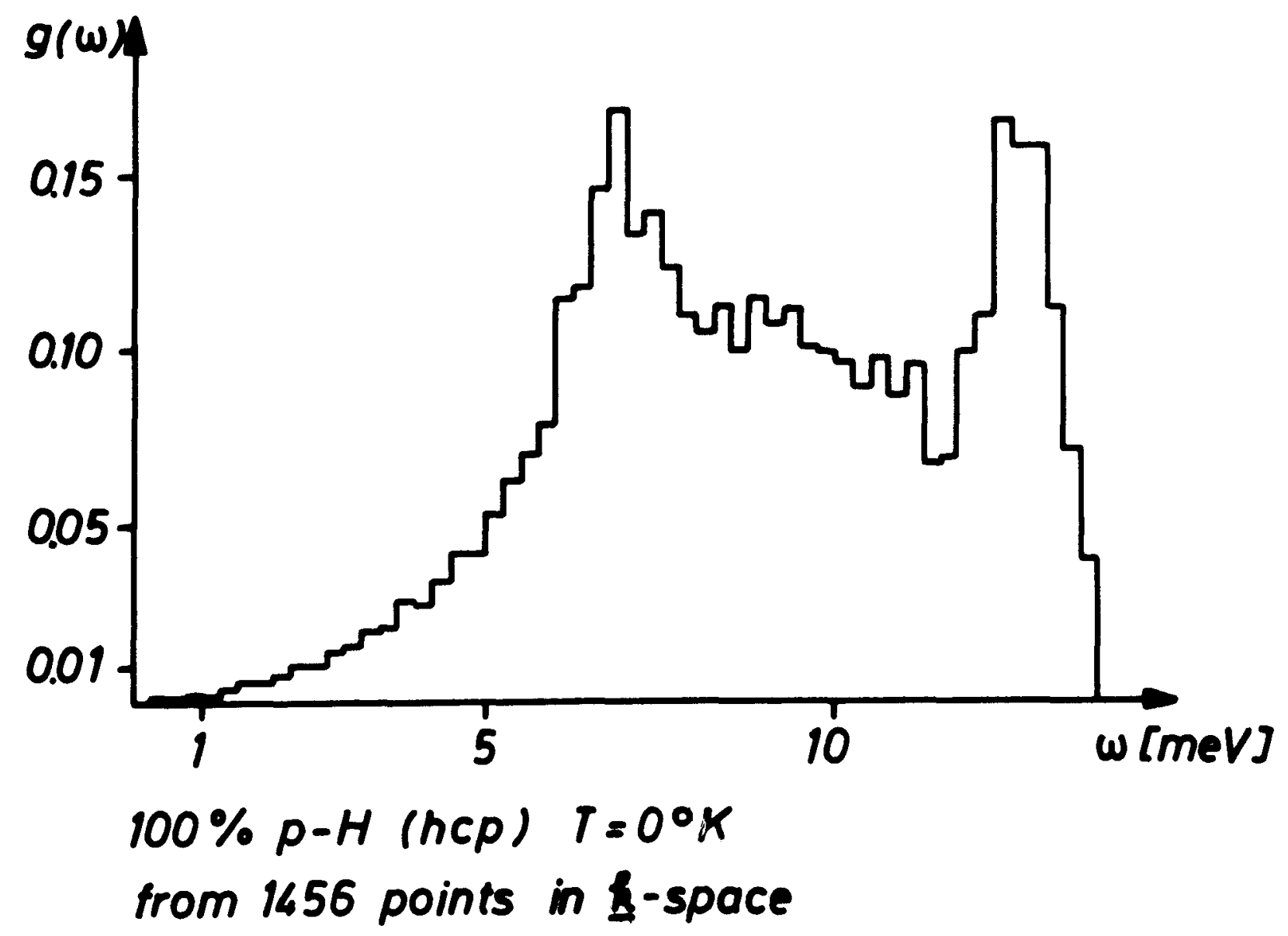

